\title{
Prismatic VHTR Benchmark Problems
}

\author{
Kevin John Connolly and Farzad Rahnema \\ Nuclear and Radiological Engineering and Medical Physics Programs \\ George W. Woodruff School \\ Georgia Institute of Technology \\ Atlanta, Ga., United States \\ connolly@gatech.edu; farzad@gatech.edu \\ Pavel V. Tsvetkov \\ Department of Nuclear Engineering \\ Texas A\&M University \\ College Station, Texas, United States
}

Corresponding Author:

Farzad Rahnema, Professor and Chair

Nuclear \& Radiological Engineering and Medical Physics Programs

George W. Woodruff School

Georgia Institute of Technology

770 State Street, Atlanta, GA 30332

Tel: 404-451-4686

Email: farzad@gatech.edu

Number of page (including this page): 56

Number of figures: 15

Number of tables: 13 


\begin{abstract}
This paper aims to fill an apparent scarcity of benchmarks based on high temperature gas-cooled reactors. Within is a description of a whole prismatic VHTR core in its full heterogeneity and modeling using continuous energy nuclear data at a representative hot operating temperature. Also included is a core which has been simplified for ease in modeling while attempting to preserve as faithfully as possible the neutron physics of the core. Fuel and absorber pins have been homogenized from the particle level, however, the blocks which construct the core remain strongly heterogeneous. A six group multigroup (discrete energy) cross section set has been developed via Monte Carlo using the original heterogeneous core as a basis.

Several configurations of the core have been solved using these two cross section sets; eigenvalue results, block-averaged power results, and some selected pin fission density results are presented in this paper, along with the six-group cross section data, so that methods developers may use these problems as a standard reference point.
\end{abstract}

Key Words: Neutronics, HTTR, hexagonal geometry, numerical benchmark, whole-core transport, heterogeneous core. 


\section{INTRODUCTION}

The Very High Temperature Reactor (VHTR) is one of the Generation IV concepts under investigation internationally. It is a thermal reactor with a graphite core which uses helium for coolant, and boasts high outlet heat and passive safety features. It is, however, expected to present a challenge to many neutronic modeling and simulation tools, due to its complex geometry, its heterogeneity, and its relatively large active core. Of importance in the development and licensing of new reactor concepts and core designs is having valid tools and methods with which to model these cores, and to have benchmark models with which to test their validity. There is, at present, a dearth of benchmarks which model the prismatic VHTR at a level of complexity necessary to judge transport-based methods which are at the same time simpler than a whole-core fully heterogeneous Monte Carlo model. This paper provides several benchmarks based on the VHTR which, it is hoped, will be of value to the community of methods developers with their eyes on this particular core.

Contained in this paper is a description of a VHTR core in full fidelity (heterogeneous to the fuel particles), followed by a summary of how the core problem in this paper has been simplified from the actual fully heterogeneous core, a discussion of the validity of the simplifications, a complete description of the stylized VHTR core, and an explanation of the method of generating the multigroup material data. Section 4 contains the eigenvalue results for the configurations investigated, as well as a fission density map at the block level for the whole core and at the pin level for selected blocks. A brief summary and some concluding remarks are presented in Section 5.

\section{DETAILED HETEROGENEOUS BENCHMARK DESCRIPTION}

This section details the VHTR active core in full fidelity. The benchmark was designed by compiling information about the prismatic VHTR design from several sources, including earlier benchmark specifications (MacDonald et al., 2003; Gougar et al., 2010), conceptual design reports (Williams et al., 1989), and material data documents (McConn et al., 2011).

\subsection{Core Geometry and Specifications}

The core is composed of 14 levels of regular hexagonal prismatic graphite blocks. Each level is made up of seven rings of whole blocks around a center column, and a ring of partial graphite blocks around the core periphery. A depiction of the basic core configuration is given by Fig. 1, in which fuel blocks are colored in yellow, standard graphite reflector blocks are in gray, and structural graphite blocks around the periphery are shaded a darker gray. The green colored regions represent the location of control channels. Note that the channels through the fuel blocks are not actually used for control rods, but as a channel for reserve shutdown material; they are modeled as empty channels containing only helium for all configurations in this benchmark.

While an attempt was made to model the core as faithfully as possible to the specifications included in design documents, some deviations included changing the shape of the core barrel from cylindrical to hexagonal prismatic, and changing the location of some control rods so that 
the whole core would have one-twelfth symmetry. The eigenvalue of the symmetric core was not found to be significantly different from the original asymmetric core for the purpose of the development of a benchmark for numerical methods development. These changes were made in order to simplify the modeling while preserving the neutron physics within the active core as accurately as possible.

Above the top and below the bottom of the core, as well as on the outside edge, a boundary condition is given as vacuum (no reentrant flux). Where sections of the core have been modeled along the lines of symmetry, a specular reflective boundary condition has been simulated.

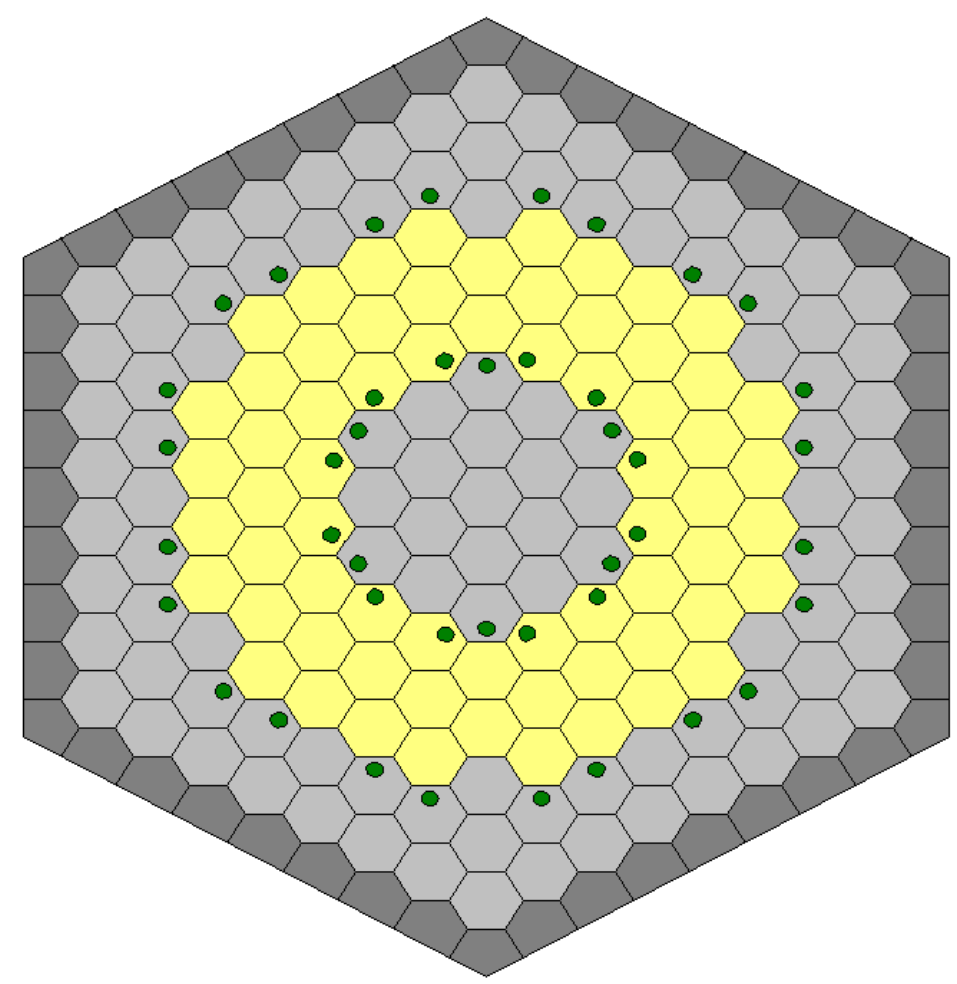

Figure 1. Whole core configuration

Table 1. Geometry parameters

\begin{tabular}{|c|c|}
\hline Number of fuel columns & 66 \\
\hline Number of control rod columns & 30 \\
\hline Number of standard reflector columns & 73 \\
\hline Number of structural reflector columns & 42 sides +6 corners \\
\hline Number of axial layers (in 3D problem) & 14 \\
\hline Number of layers in active core (3D problem) & 10 \\
\hline Flat-to-flat core width & $519.6 \mathrm{~cm}$ \\
\hline Flat-to-flat block width & $36 \mathrm{~cm}$ \\
\hline Block height (in 3D problem) & $79.3 \mathrm{~cm}$ \\
\hline
\end{tabular}




\begin{tabular}{|c|c|}
\hline Number of fuel pins in standard block & 210 \\
\hline Number of fuel pins in reserve shutdown block & 186 \\
\hline Fuel pin hole diameter & $1.27 \mathrm{~cm}$ \\
\hline Number of absorber pins in fuel block & 6 \\
\hline Absorber pin hole diameter & $1.27 \mathrm{~cm}$ \\
\hline Number of large coolant channels in standard block & 102 \\
\hline Number of large coolant channels in reserve shutdown block & 88 \\
\hline Number of small coolant channels in standard block & 6 \\
\hline Number of small coolant channels in reserve shutdown block & 7 \\
\hline Large coolant channel diameter & $1.588 \mathrm{~cm}$ \\
\hline Small coolant channel diameter & $1.27 \mathrm{~cm}$ \\
\hline Pin cell pitch in fuel block & $1.8796 \mathrm{~cm}$ \\
\hline Coolant channel pitch in cooled reflector block & $3.2556 \mathrm{~cm}$ \\
\hline Reserve shutdown channel diameter & $9.525 \mathrm{~cm}$ \\
\hline Control channel diameter / outer control rod diameter & $9.525 \mathrm{~cm}$ \\
\hline Inner control rod diameter & $5.28 \mathrm{~cm}$ \\
\hline Distance from control rod center to block center & $9.756 \mathrm{~cm}$ \\
\hline
\end{tabular}

Each block within the core (but not in the peripheral graphite ring) has the same dimensions of $36 \mathrm{~cm}$ from flat edge to flat edge. Additional geometry information is provided in Table 1. A thorough description of each block's composition follows.

\subsection{Block Geometry and Specifications}

Each block in the active core region has a flat-to-flat width of $36 \mathrm{~cm}$, and a height of $79.3 \mathrm{~cm}$. The fundamental block geometry of each type of block is shown in Figures 2-4, in which the graphite structure is represented by gray, the fuel pin regions are shown in pale yellow, burnable absorber pin regions are shaded green, and the control rod areas are colored deep red. The white spaces in the figures represent helium coolant. Fuel pin, absorber pin, and control rod regions are heterogeneous and more description follows for each. 

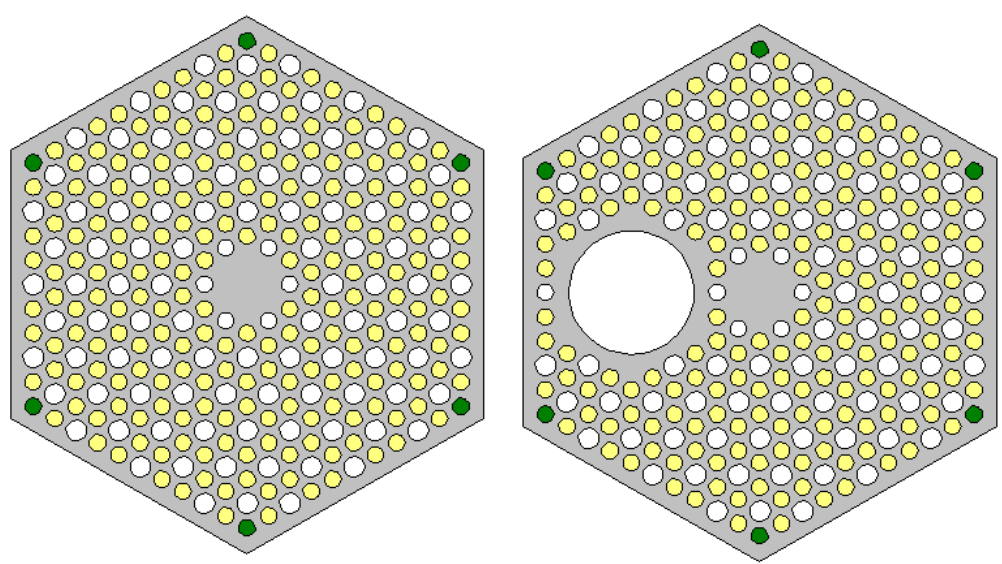

Figure 2. Standard fuel block, fuel block with reserve shutdown channel.
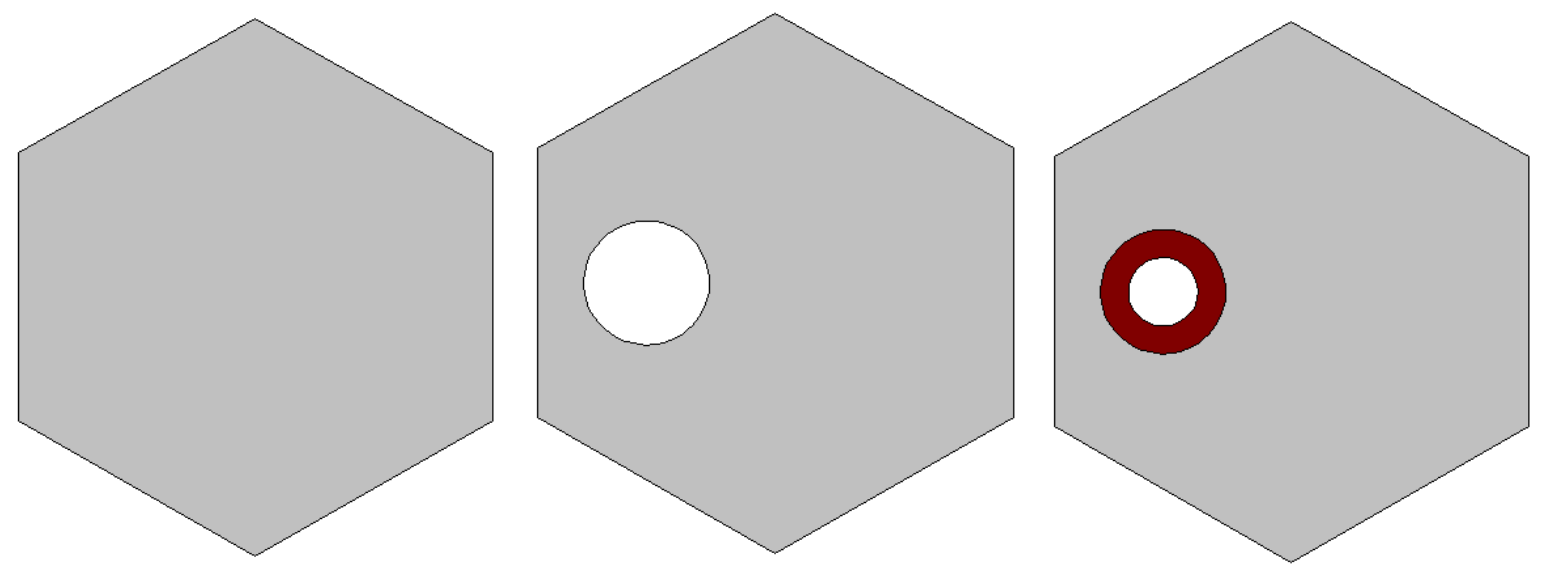

Figure 3. Standard reflector block, control block with control rod absent, control block.
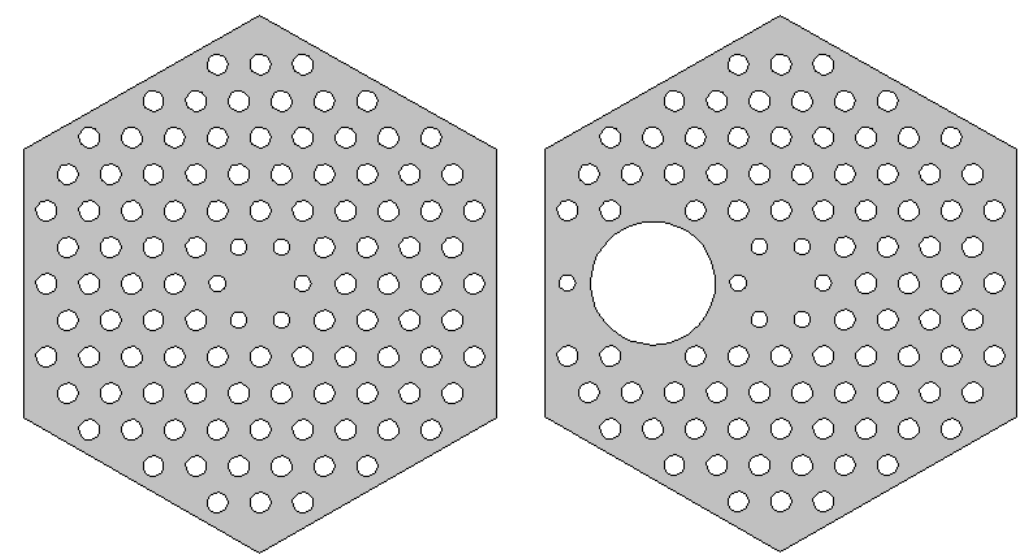

Figure 4. Standard cooled reflector block, cooled reflector with reserve shutdown channel. 
Fuel blocks contain 210 holes of radius $0.635 \mathrm{~cm}$ for fuel compacts, 102 holes of radius 0.794 $\mathrm{cm}$ for coolant to flow through, and 6 smaller coolant holes of $0.635 \mathrm{~cm}$ radius. In addition, six holes for burnable absorbers are at the corners of the fuel blocks, and are of the same radius as the fuel pins. The holes are arranged to have a pitch of $1.8796 \mathrm{~cm}$.

Fuel blocks for which a reserve shutdown channel has been drilled are similar to standard fuel blocks, but they have a hole of $9.525 \mathrm{~cm}$ diameter located $9.756 \mathrm{~cm}$ from the center of the block. Furthermore, these blocks have only 186 fuel holes, 88 larger coolant holes, and 7 smaller coolant holes of the same dimensions as those in the standard fuel block. The burnable absorber pins remain the same.

In the fuel pins, uranium oxycarbide $\left(\mathrm{UO}_{1.5} \mathrm{C}_{0.5}\right.$, with uranium enriched to $15.5 \%$ in ${ }^{235} \mathrm{U}$ by weight) fuel spheres of radius $0.02125 \mathrm{~cm}$ are enveloped in four layers of material: a carbon buffer $0.01 \mathrm{~cm}$ thick, a pyrolitic carbon layer $0.0035 \mathrm{~cm}$ thick, $0.0035 \mathrm{~cm}$ of silicon carbide, and an outer layer of thickness $0.004 \mathrm{~cm}$ consisting of pyrolitic carbon. These are placed in a graphite matrix with a packing fraction of 35\%, and are arranged in a cubic lattice for ease of modeling, as shown in Figure 5. The side length of the cubic lattice elements is $0.09664222 \mathrm{~cm}$. A similar approach is taken for the burnable absorber pins, where boron carbide reactivity control spheres of radius $0.01 \mathrm{~cm}$ are encased in carbon layers of $0.0018 \mathrm{~cm}$ and $0.0023 \mathrm{~cm}$ thickness. These, too, are modeled in a cubic lattice (of side length $0.04758168 \mathrm{~cm}$ ) in this benchmark specification rather than their realistically more random distribution. Although the reference suggests varying concentrations of absorbing material distributed in the core as a reactivity control, no more detail is given. For the sake of this benchmark, the burnable absorber material is uniform wherever it appears. Fuel and poison pins are both placed in holes in the graphite block, with a $0.0125 \mathrm{~cm}$ gap surrounding the fuel compacts making up the pin, and a $0.0635 \mathrm{~cm}$ gap around the poison compacts. Graphite plugs are inserted into the holes to keep the compacts in place. The graphite plugs in fuel cells are each $2.69 \mathrm{~cm}$ long, of the same diameter as the fuel hole, and placed at the top and bottom of each block. The remaining 73.92 $\mathrm{cm}$ of the fuel pin region (between the plugs) is the heterogeneous fuel compact. The burn pins are structured similarly, but the graphite plugs at the top and bottom of the absorber holes are each $3.558 \mathrm{~cm}$ in height. 

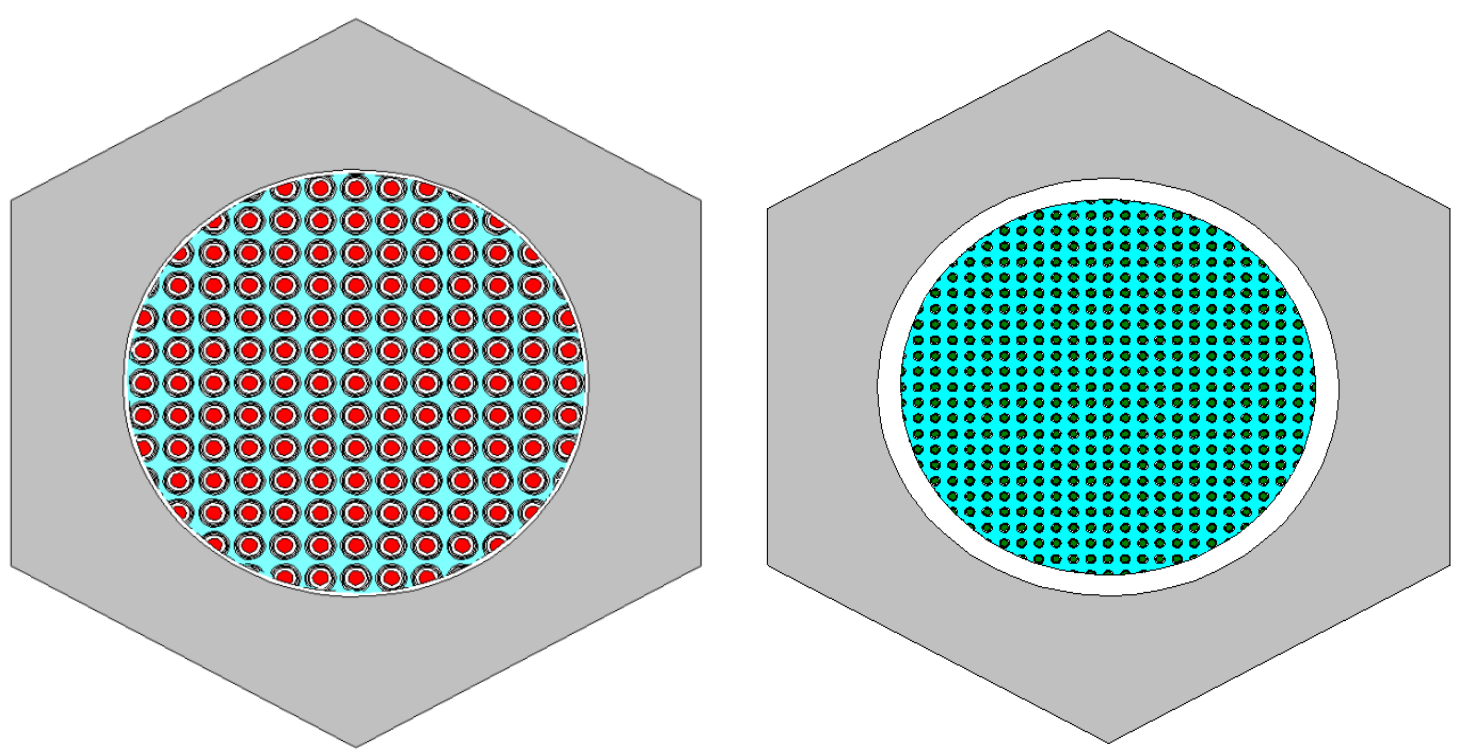

Figure 5. Cut-through, fuel pin cell and burnable absorber pin cell.

Reflector blocks are composed of the same graphite as the fuel blocks. They may have control channels within. Above and below the active core, reflector blocks contain the same coolant channels as those which run through the standard fuel blocks. Control rods and holes are of the same dimensions as the reserve shutdown holes found in fuel blocks.

Table 2: Materials used in benchmark

\begin{tabular}{|c|c|c|c|}
\hline Material & \multicolumn{2}{|c|}{ Isotopic Composition } & Density $\left(\mathrm{g} / \mathrm{cm}^{3}\right)$ \\
\hline \multirow{4}{*}{ Fuel kernel } & ${ }^{235} \mathrm{U}$ & 0.0522 & \multirow{4}{*}{10.5} \\
\hline & ${ }^{238} \mathrm{U}$ & 0.2811 & \\
\hline & C-nat & 0.1667 & \\
\hline & ${ }^{16} \mathrm{O}$ & 0.5000 & \\
\hline $\begin{array}{l}\text { Carbon buffer (in fuel } \\
\text { particle) }\end{array}$ & C-nat $(*)$ & 1.0 & 1.0 \\
\hline $\begin{array}{l}\text { Pyrolitic carbon (in } \\
\text { fuel particle) }\end{array}$ & C-nat $(*)$ & 1.0 & 1.9 \\
\hline \multirow{2}{*}{ Silicon carbide } & C-nat & 0.5 & \multirow{2}{*}{3.2} \\
\hline & ${ }^{28} \mathrm{Si}$ & 0.5 & \\
\hline Matrix graphite & C-nat $(*)$ & 1.0 & 0.94 \\
\hline Graphite plugs & C-nat $(*)$ & 1.0 & 1.74 \\
\hline \multirow{3}{*}{ Poison kernel } & ${ }^{10} \mathrm{~B}$ & 0.3146 & \multirow{3}{*}{2.47} \\
\hline & ${ }^{11} \mathrm{~B}$ & 0.0318 & \\
\hline & C-nat & 0.6536 & \\
\hline $\begin{array}{l}\text { Pyrolitic carbon (in } \\
\text { poison particle) }\end{array}$ & C-nat(*) & 1.0 & 1.87 \\
\hline
\end{tabular}




\begin{tabular}{|c|c|c|c|}
\hline \multirow{3}{*}{ Control rod } & ${ }^{10} \mathrm{~B}$ & 0.3146 & \multirow{3}{*}{1.23573} \\
\hline & ${ }^{11} \mathrm{~B}$ & 0.0318 & \\
\hline & C-nat(*) & 0.6536 & \\
\hline \multirow{10}{*}{ Incoloy-800 } & C-nat & 0.002984 & \multirow{10}{*}{7.94} \\
\hline & ${ }^{27} \mathrm{Al}$ & 0.007663 & \\
\hline & ${ }^{28} \mathrm{Si}$ & 0.012760 & \\
\hline & ${ }^{32} \mathrm{~S}$ & 0.000172 & \\
\hline & ${ }^{48} \mathrm{Ti}$ & 0.004319 & \\
\hline & ${ }^{52} \mathrm{Cr}$ & 0.222681 & \\
\hline & ${ }^{55} \mathrm{Mn}$ & 0.009785 & \\
\hline & ${ }^{56} \mathrm{Fe}$ & 0.430100 & \\
\hline & ${ }^{58} \mathrm{Ni}$ & 0.305302 & \\
\hline & ${ }^{63} \mathrm{Cu}$ & 0.004234 & \\
\hline \multirow{2}{*}{ Block graphite } & C-nat(*) & 0.9999998 & \multirow[b]{2}{*}{1.74} \\
\hline & ${ }^{10} \mathrm{~B}$ & 0.0000002 & \\
\hline \multirow{2}{*}{$\begin{array}{l}\text { Outer (structural) } \\
\text { reflector }\end{array}$} & C-nat(*) & 0.9999998 & \multirow{2}{*}{1.78} \\
\hline & ${ }^{10} \mathrm{~B}$ & 0.0000002 & \\
\hline Helium coolant & ${ }^{4} \mathrm{He}$ & 1.0 & 0.0001786 \\
\hline
\end{tabular}

*Natural carbon is in the form of graphite in these materials; $S(\alpha, \beta)$ data should be used.

The control rod regions' outer diameter is $9.525 \mathrm{~cm}$, thus leaving no gap in the model between the control material and the graphite. They have an inner diameter of $5.28 \mathrm{~cm}$ surrounding a region of vacuum through which, for the sake of the model, helium coolant flows. The control rods themselves are of boron carbide and structural carbon, and are annular with the inner diameter of $5.28 \mathrm{~cm}$ and the outer diameter $8.26 \mathrm{~cm}$. The outer annular structure for the control rod composes the balance of the control region $(8.26 \mathrm{~cm}$ inner diameter; $9.525 \mathrm{~cm}$ outer diameter) and is constructed of Incoloy-800.

A listing of all materials used for the benchmark description is presented in Table 2.

\section{STYLIZED BENCHMARK DESCRIPTION}

In this section, a description is given of the benchmark problem, explaining how it differs from the fully heterogeneous VHTR design and giving rationale for these simplifications. The development of the problem is detailed, including an explanation and justification of the cross section generation method. A complete specification is then given of the new benchmark problem.

\subsection{Stylized Core Model Development}

The purpose of this work has been to reduce the complexity of the problem specification while retaining as many details as possible to produce a valid representation of the overall core neutronic behavior. As this benchmark is intended to serve as a reference to developers of 
methods which model block-level heterogeneity, the aim has been to create an accessible benchmark with enough detail to present a challenging test to a new method, but simplified enough so as to be useful; for instance, very few methods, at present, resolve pin-level heterogeneity in their full-core calculations, and a problem which maintains such minute detail is unlikely to serve much immediate purpose to the community. A similar approach was taken with a benchmark in the style of Japan's High Temperature Test Reactor (Zhang et al., 2011), which has been a valuable model for methods testing (Yang and Satvat, 2012).

The core model neutronic behavior was simulated using the stochastic neutron transport method MCNP5 (X-5 Monte Carlo Team, 2005). The ENDF/B-VII continuous energy library at 900K was selected for the model as a typical hot operating temperature. For the graphite throughout the core and for the uranium and oxygen in the fuel, $S(\alpha, \beta)$ data was used at $800 \mathrm{~K}$, as this was the closest temperature available to the hot operating temperature chosen. A set of regions within the core was defined for multigroup macroscopic cross section generation using MoCSGen, a Monte Carlo-based multigroup macroscopic cross section utility (Ilas, 2002; Pounders et al., 2007; Pounders et al., 2014) which generates flux-weighted homogenized cross sections. A 6-group library including a $\mathrm{P}_{1}$ scattering kernel, whose energy bounds may be found in Table 3 , was created for the core. The bounds were chosen as they have appeared previously in the analysis of prismatic high temperature reactors (Murata et al. 1994; Zhang et al., 2011). While cross section libraries with a greater number of energy groups are commonly used, the inclusion of the corresponding cross section data would be too cumbersome to include in this paper due to size limitations. Additional discussion of the method of cross section generation is beyond the scope of this paper.

Table 3. 6 group energy structure

\begin{tabular}{|c|c|}
\hline Group & Upper energy $(\mathrm{MeV})$ \\
\hline 1 & 20 \\
\hline 2 & 0.183 \\
\hline 3 & $9.61 \times 10^{-4}$ \\
\hline 4 & $2.38 \times 10^{-6}$ \\
\hline 5 & $6.5 \times 10^{-7}$ \\
\hline 6 & $1.05 \times 10^{-7}$ \\
\hline
\end{tabular}

From two core configurations, a total of fourteen unique materials were defined within the core. The first configuration was a one twenty-fourth symmetric core (one twelfth radial symmetry and one half axial symmetry) with control material absent. From this configuration, three fuel material zones were established, five graphite material zones, one for burnable absorber pins, and one for helium coolant. Figure 6 depicts an axial cross-section of the core with different material regions indicated. The second configuration was the core with some rods in. From the one twenty-fourth symmetric core, control rods were placed in the three axial regions closest to the central line of symmetry. Although this is not a realistic depiction of the insertion of control 
material, it was considered a reasonable model for generating cross sections. This model yielded an additional fuel material specification, two more graphite regions, and a definition for control material. Figure 7 illustrates an axial slice of the second configuration simulated.

All of the materials present were homogenized from their original heterogeneity to become uniform regions. Fuel regions were homogenized from their initial specification, which included uranium oxycarbide fuel at $15.5 \%$ weight enrichment encapsulated in pyrolitic carbon and silicon carbide layers embedded in a graphite matrix, graphite plugs at the top and bottom of fuel compact stacks, and a gap surrounding the compacts. Burnable absorber regions were similarly homogenized from gap, plug, and absorber compacts. Graphite regions and helium coolant regions were already homogeneous from a material specification perspective, but both were given a uniform cross-section throughout at least individual blocks. Control material was defined as the homogenization of boron carbide control material cladded by Incoloy- 800 .

In Figure 6, the pale yellow color denotes inner fuel blocks. The inner fuel and inner graphite composition were determined by homogenizing all of the fuel regions and all of the graphite regions, respectively, in these regions using MoCS-Gen. The same procedure was used for the middle fuel and graphite regions, which were those in the light blue blocks, and the outer fuel and graphite regions, which were those in those blocks colored in light green. All of the burnable absorber pins in the core were homogenized into a single region for cross section generation, and the same was done for all of the helium coolant present within the core. Graphite cross sections were also tabulated for the three different graphite regions illustrated in Figure 6, although upon testing, the central reflector regions and outer reflector regions, indicated by the pale and medium shades of gray, were found to be interchangeable, and only the graphite cross section determined by the homogenization of the medium gray blocks is kept for this benchmark. Finally, a material region was defined for the core periphery, which represents the structural permanent graphite blocks along the edge of the core. This region is colored in the darker shade of gray.

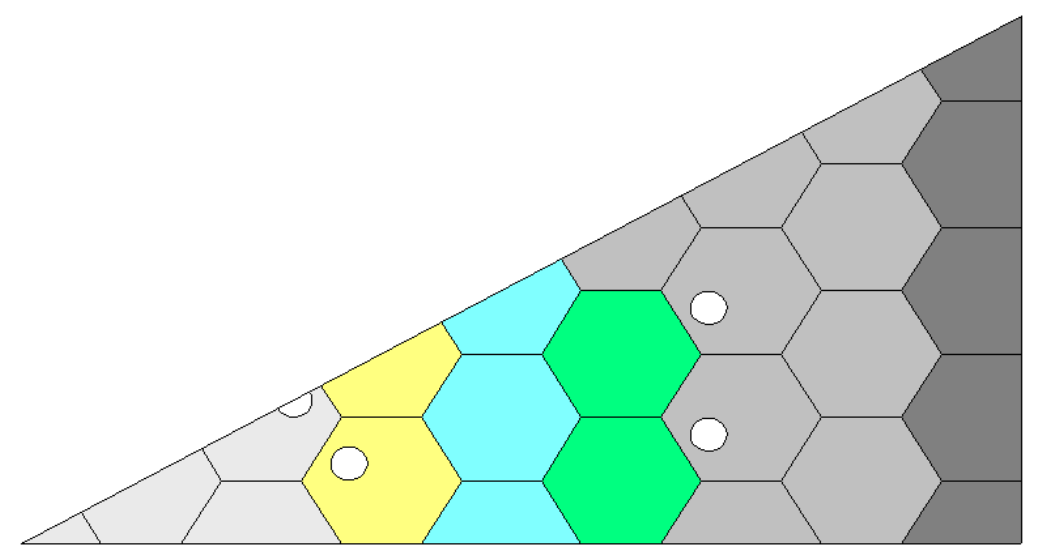

Figure 6. 1/12 of core with all rods out, cross section regions indicated in text 


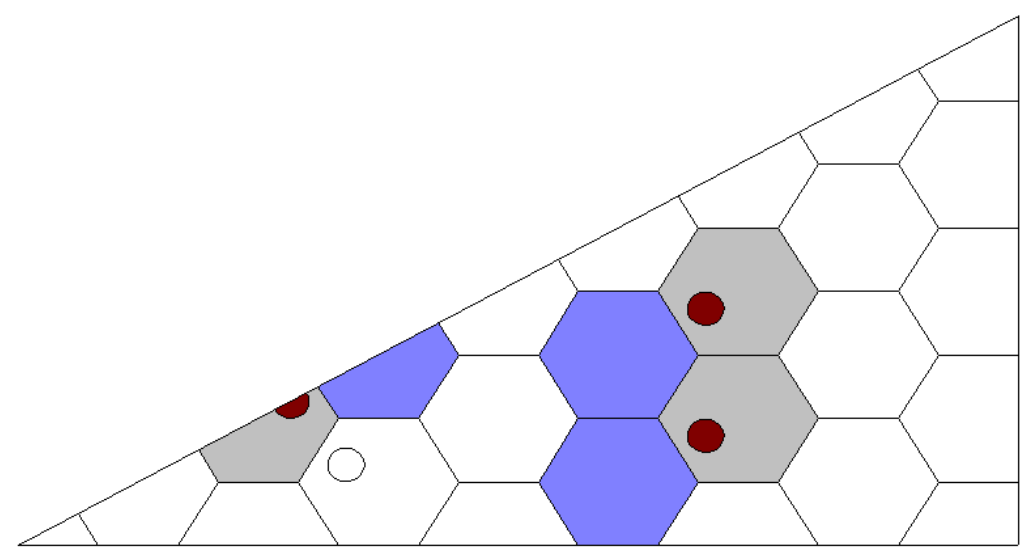

Figure 7. 1/12 of core with rods in, cross section generation regions indicated in text

The second core model used for cross section generation is illustrated in Figure 7. Only the regions of interest are colored in this figure. Material cross section data was determined for the fuel and graphite in those blocks adjacent to the inserted control rods, which are colored in lavender. A final graphite set was found for the graphite in control blocks, which are shown in gray. The control rods are shown in brown, and represent the homogenization of the control material and cladding; they remain annular in the benchmark specification. The blocks without shading were not of interest for development of homogeneous material regions.

\subsection{Geometry specification}

The core and block specification is exactly the same in the stylized core as that described for the fully heterogeneous core. The only difference in the active core region is that fuel and poison pin regions have been homogenized so that everything inside of the fuel pin hole defined in Table 1 is homogenized fuel material, and everything within the burnable absorber hole is homogenized burnable absorber. The only other difference is that Incoloy-800 and control material have been homogenized to form a single annular control material region of the inner and outer diameter given by Table 1.

\section{RESULTS}

This section includes results from the continuous energy heterogeneous core, the stylized 3-D core, and a stylized 2-D version of the core. All results are from core models utilizing core symmetry; that is, they model a $1 / 12$ radial section and, for the 3-D configurations, a $1 / 2$ axial section. Eigenvalue results are given for all core configurations with their corresponding uncertainty. Fission density results are also presented. Because of the large number of pins in the core, it would be impractical to list values for all individual pins; therefore, block-level fission density results are given for all blocks in each core. Data is presented in tables using the block numbering scheme presented in Figure 8. 


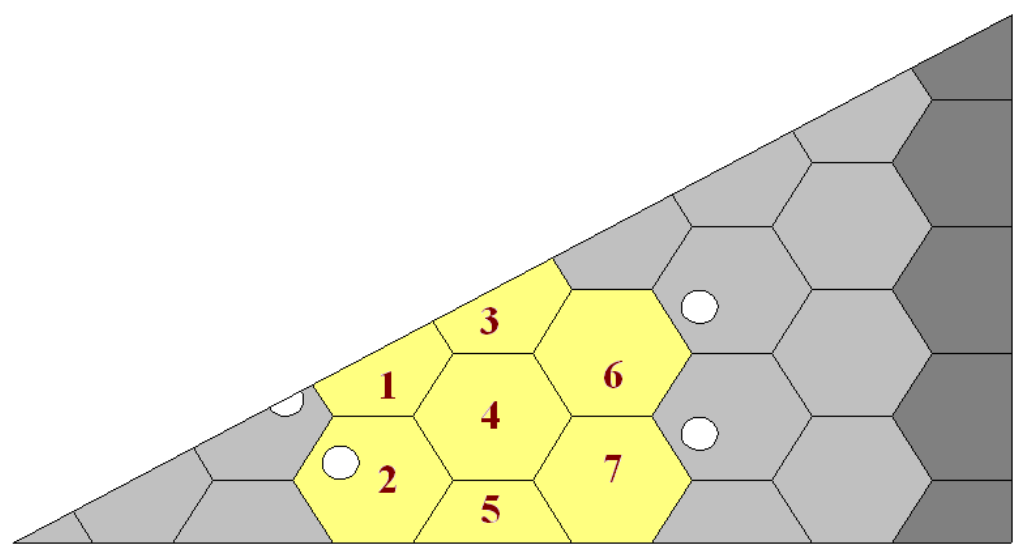

Figure 8. Block numbering scheme

Fission density values have been normalized such that a value of 1 corresponds to the core average block power.

\subsection{Continuous energy heterogeneous core}

The full fidelity continuous energy core was modeled with all rods out and all rods in using 1/24 symmetry, as the cores are axially symmetric as modeled. MCNP5 was used for the calculations, in which 125,000 particles were simulated per cycle, and 4,000 active cycles were simulated after an initial 200 inactive cycles. A full core calculation was done to produce converged fission source data, and another full core calculation was done to tally fission density information. The core calculation for tallying block-level fission density data in the core with all rods out required just over 2 days on 40 processors, or 136440 cpu minutes; that for all rods in required 136640 cpu minutes, which represents 2.3 days on 40 processors.

Eigenvalue results for all cores are given in Table 4, along with their corresponding uncertainty values, in pcm, as determined by MCNP.

Table 4. Eigenvalue results for all cores: $k_{\text {eff }}(\sigma$ in $\mathbf{~ c m})$

\begin{tabular}{|c|c|c|c|}
\hline & Heterogeneous, CE & 3D Stylized, 6g & 2D Stylized, 6g \\
\hline All Rods Out (ARO) & $1.06611( \pm 4 \mathrm{pcm})$ & $1.09201( \pm 3 \mathrm{pcm})$ & $1.09844( \pm 2 \mathrm{pcm})$ \\
\hline Some Rods In (SRI) & $\mathrm{n} / \mathrm{a}$ & $1.00769( \pm 3 \mathrm{pcm})$ & $1.04655( \pm 2 \mathrm{pcm})$ \\
\hline All Rods In (ARI) & $0.91538( \pm 4 \mathrm{pcm})$ & $0.94894( \pm 3 \mathrm{pcm})$ & $0.95442( \pm 2 \mathrm{pcm})$ \\
\hline
\end{tabular}

Table 5 contains the block-averaged fission density values for all of the blocks in the heterogeneous core. These values have been normalized such that the average block in the core has a fission density value of 1 . For the case with all rods out, the uncertainty in the fission density values ranged from $0.03 \%$ to $0.06 \%$. With all rods in, the fission density uncertainty 
values ranged from $0.03 \%$ to $0.07 \%$. Pin fission density results for the fully heterogeneous continuous energy cores are not included, as the reader may calculate these based on the specification detailed in the previous section. In the following section, fission density values are provided at the pin level for the stylized multigroup cores, as these are expected to be useful for benchmarking multigroup transport methods.

Table 5. Block-averaged fission density values, continuous energy heterogeneous core

\begin{tabular}{|c|c|c|c|c|c|c|c|}
\hline Block & 1 & 2 & 3 & 4 & 5 & 6 & 7 \\
\hline \multicolumn{7}{|c|}{ All Rods Out } \\
\hline 1,10 & 0.6643 & 0.6922 & 0.5961 & 0.6210 & 0.6312 & 0.5300 & 0.5539 \\
\hline 2,9 & 0.9369 & 0.9779 & 0.8437 & 0.8760 & 0.8892 & 0.7537 & 0.7860 \\
\hline 3,8 & 1.1563 & 1.2062 & 1.0429 & 1.0819 & 1.0983 & 0.9332 & 0.9712 \\
\hline 4,7 & 1.3127 & 1.3698 & 1.1825 & 1.2277 & 1.2461 & 1.0578 & 1.1017 \\
\hline 5,6 & 1.3938 & 1.4550 & 1.2551 & 1.3039 & 1.3245 & 1.1240 & 1.1703 \\
\hline \multicolumn{7}{|c|}{ All Rods In } \\
\hline 1,10 & 0.5752 & 0.6158 & 0.5471 & 0.5893 & 0.6116 & 0.3953 & 0.4482 \\
\hline 2,9 & 0.9020 & 0.9680 & 0.8576 & 0.9224 & 0.9560 & 0.6216 & 0.7030 \\
\hline 3,8 & 1.1651 & 1.2504 & 1.1070 & 1.1922 & 1.2358 & 0.8030 & 0.9091 \\
\hline 4,7 & 1.3521 & 1.4506 & 1.2860 & 1.3828 & 1.4340 & 0.9324 & 1.0553 \\
\hline 5,6 & 1.4484 & 1.5531 & 1.3765 & 1.4806 & 1.5358 & 0.9976 & 1.1302 \\
\hline
\end{tabular}

\subsection{Stylized cores, 6 group library}

Solutions were found for the stylized six group core in three configurations. MCNP was used to construct the solution, using the 6-group discrete energy macroscopic cross section library in Appendix A. The first configuration, all rods out (ARO), was first solved, as with the heterogeneous configuration, to generate a converged fission source. The same parameters of 125,000 particles per cycle and 200 inactive cycles followed by 4,000 active cycles were used for all of the six group calculations. As with the fully heterogeneous continuous energy case, the stylized 6 group cores utilized 1/24 symmetry: 1/12 rotational symmetry, and $1 / 2$ axial symmetry.

It may be noted from the eigenvalue results presented in Table 4 that the $\mathrm{k}_{\mathrm{eff}}$ values determined using the multigroup cross section library differ from those of the continuous energy problem by approximately 2500 pcm (2.5\%). This was considered acceptable for the sake of this paper, in that its purpose is to provide numerical benchmarks for developers of transport methods, and is not intended to be a study in cross section generation. However, it is worth stating that the means of homogenizing fuel particles and pins and generating a few group macroscopic cross section set used in the compilation of these benchmark problems has preserved the physics of the actual core far more closely than a volume homogenization approach; simple volume homogenization methods may lead to eigenvalue errors of approximately $10 \%$. Additional discussion of methods for the generation of multigroup homogenized cross sections in highly heterogeneous cores is beyond the scope of this paper. 
The layout of the ARO core is illustrated in Fig. 9, in which the yellow assemblies represent those for which the "inner fuel block" fuel and graphite composition was used, the pale aqua blocks signify "middle fuel block" fuel and graphite compositions, and the green blocks represent those for which "outer fuel block" fuel and graphite should be used. All of the reflector blocks use the same graphite composition except the ring of permanent structural blocks at the edge which are depicted in darker gray; these were modeled in the "permanent reflector" graphite composition. The ARO core features 5 levels of blocks arranged just as shown by Fig. 9, with two layers above where the fuel blocks are replaced with cooled reflector blocks; the cooled reflector blocks are composed of the same graphite as the standard reflector blocks. Below the modeled active core is a specular reflective boundary condition. The ARO core calculation completed after 22337 cpu minutes.

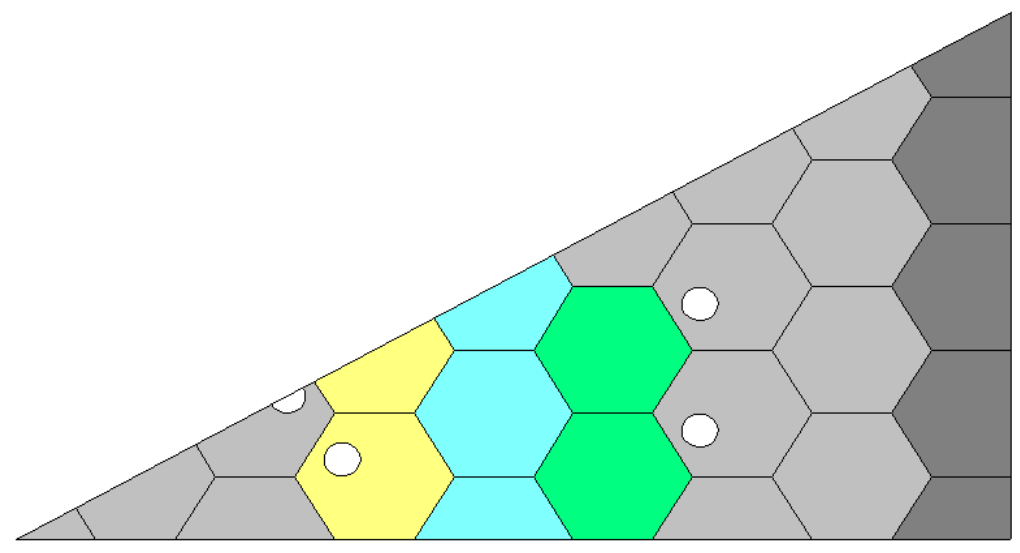

Figure 9. All rods out block diagram

The configuration with some rods in (SRI) is described here to be a numerical benchmark which challenges solution methods and does not strive for realism. The control rods are present in the middle of the active core, but not the top and bottom, thus creating a model which has the flux peaked at the top and bottom of the core, depressed flux in the center of the reactor, and a nonphysical state of control existing. The three axial layers at the center of the core are identical to the standard ARI configuration as illustrated by Figure 10. The axial layer of the active core at the very top and bottom has all rods out, as illustrated by Figure 9. The two axial levels of reflector also have no control material present. The region of the active core which is either second or ninth from the top (or bottom, since axial reflection exists at the center) of the active core is as shown in Figure 11. In Figures 10 and 11, the same color scheme is followed as for Figure 9, but with the following additions: lavender blocks represent fuel blocks with "control block adjacent" fuel and graphite compositions, the rust-colored circles represent control rods, and blocks where control rods are present should use "control block graphite" instead of the standard graphite reflector block cross section set. The SRI core required 23985 minutes of computing time to reach its solution. 
The third core configuration has all rods in (ARI). In this configuration, all layers of the axial core are illustrated as in Figure 10. The two layers above the active core have control rods and blocks in the same place as in the active core, but cooled reflector blocks are used in place of fuel blocks. Even for cooled reflector blocks positioned next to control rods, the standard "reflector block graphite" cross section set is used rather than the "control adjacent" set in order to keep a single composition for all cooled reflector blocks; as these are outside of the active core, the effect on reactivity is expected to be negligible for the purposes of presenting a numerical benchmarking description. The ARI core calculation required 19879 cpu minutes.

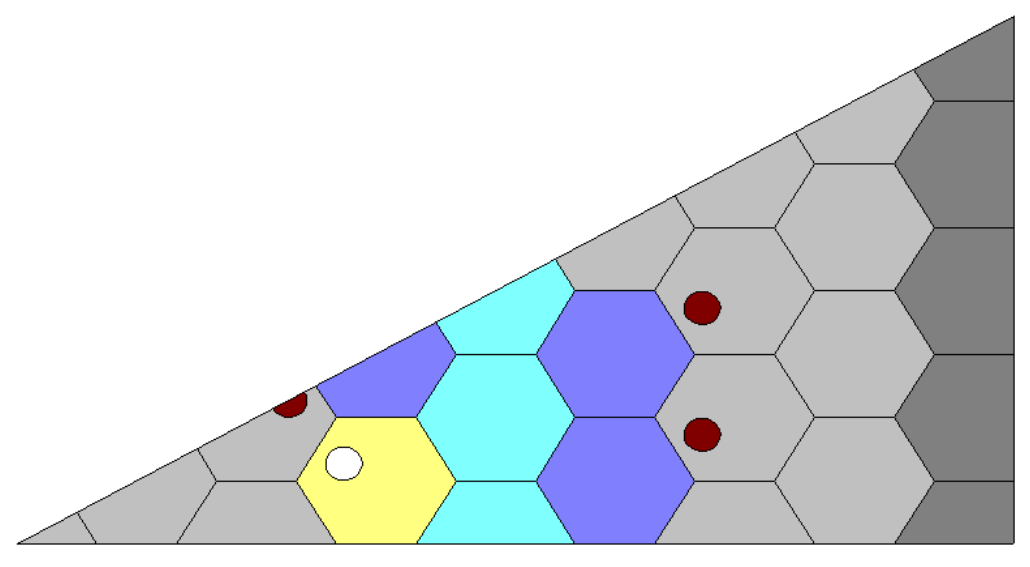

Figure 10. All rods in block diagram

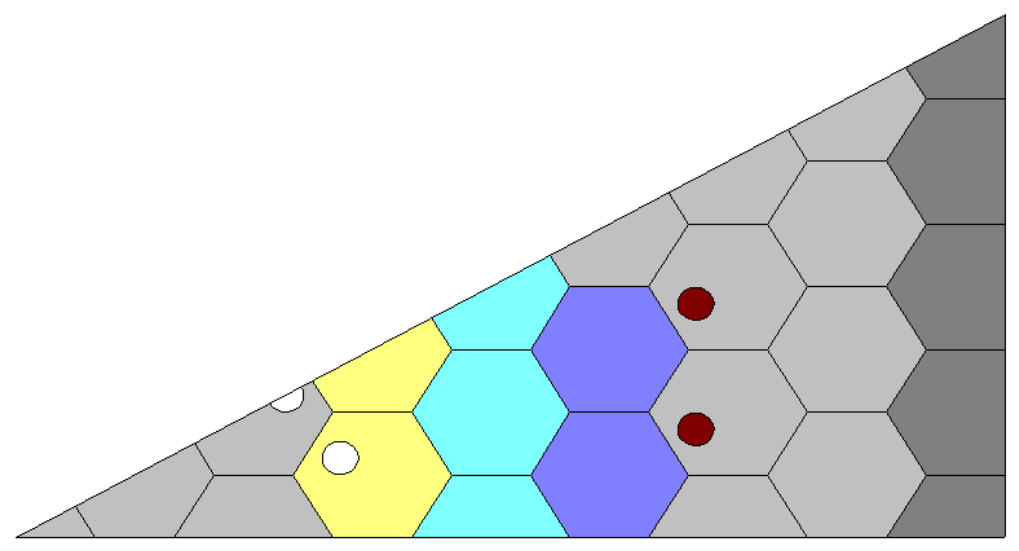

Figure 11. Block diagram for some rods in, 3-D core, axial layers 2, 9

The block-averaged fission density values for all three stylized cores are presented in Table 6 . For both the ARI and ARO cores, the uncertainty in the fission density values ranged from 
$0.02 \%$ to $0.04 \%$. In the more sharply-peaked SRI core, the uncertainty ranged from $0.01 \%$ at the high-flux regions to $0.07 \%$ in some of the blocks in the heavily-controlled part of the core.

Values are normalized such that the average block fission density in the core is 1.0.

Table 6. Block-averaged fission density values, 3-D stylized cores

\begin{tabular}{|c|c|c|c|c|c|c|c|}
\hline Block & 1 & 2 & 3 & 4 & 5 & 6 & 7 \\
\hline \multicolumn{7}{|c|}{ All Rods Out } \\
\hline 1,10 & 0.6898 & 0.8057 & 0.5861 & 0.5631 & 0.5718 & 0.6457 & 0.6147 \\
\hline 2,9 & 0.9032 & 1.0662 & 0.7697 & 0.7294 & 0.7396 & 0.8696 & 0.8174 \\
\hline 3,8 & 1.1183 & 1.3209 & 0.9527 & 0.9030 & 0.9163 & 1.0775 & 1.0130 \\
\hline 4,7 & 1.2699 & 1.4998 & 1.0821 & 1.0253 & 1.0408 & 1.2242 & 1.1507 \\
\hline 5,6 & 1.3470 & 1.5912 & 1.1483 & 1.0886 & 1.1040 & 1.2996 & 1.2214 \\
\hline \multicolumn{7}{|c|}{ Some Rods In } \\
\hline 1,10 & 2.4737 & 2.8796 & 1.9932 & 1.9847 & 2.0311 & 1.9818 & 1.9598 \\
\hline 2,9 & 1.8576 & 2.2310 & 1.3863 & 1.4286 & 1.4795 & 1.0823 & 1.1500 \\
\hline 3,8 & 0.8107 & 1.0622 & 0.7415 & 0.7595 & 0.7936 & 0.5661 & 0.6114 \\
\hline 4,7 & 0.3810 & 0.4933 & 0.3684 & 0.3726 & 0.3883 & 0.2850 & 0.3072 \\
\hline 5,6 & 0.2277 & 0.2934 & 0.2220 & 0.2238 & 0.2329 & 0.1722 & 0.1856 \\
\hline \multicolumn{7}{|c|}{ All Rods In } \\
\hline 1,10 & 0.5698 & 0.7089 & 0.5623 & 0.5690 & 0.5915 & 0.4309 & 0.4674 \\
\hline 2,9 & 0.8461 & 1.0646 & 0.8378 & 0.8412 & 0.8733 & 0.6498 & 0.6994 \\
\hline 3,8 & 1.1015 & 1.3856 & 1.0894 & 1.0947 & 1.1363 & 0.8451 & 0.9103 \\
\hline 4,7 & 1.2804 & 1.6105 & 1.2677 & 1.2725 & 1.3205 & 0.9821 & 1.0573 \\
\hline 5,6 & 1.3711 & 1.7243 & 1.3575 & 1.3625 & 1.4142 & 1.0526 & 1.1327 \\
\hline
\end{tabular}

In Tables B1 through B3, found in Appendix B, pin fission density results are presented for the three 3-D core configurations. As a listing of all pins in the core would be exhaustive, both in computational time requirements and space, the only results given are for the axial layer of the core containing the highest flux and power. Calculated uncertainty in these results ranges from $0.10 \%$ to $0.17 \%$ in the ARO core, $0.05 \%$ to $0.10 \%$ in the SRI core, and $0.10 \%$ to $0.18 \%$ in the ARI core. The pin numbering scheme is illustrated by Figure 13. Results in these tables for pin fission density figures are normalized such that the average fuel pin within each individual block is 1.0. Pin fission density values normalized over all pins in the core may be found by multiplying the pin fission density value by the block-averaged fission density value in Table 6 . Note that those pins along the line of symmetry are correctly normalized.

Finally, a 2-D stylized core was created based on the 3-D stylized core. It represents, essentially, a slice of the core which extends infinitely in the axial direction. The fuel regions are identical in composition and placement to those in the 3-D core. Three configurations were solved using MCNP and using the 6-group macroscopic cross sections from Appendix A: an all-rods-out configuration, and all-rods-in core, and one with only the center rods in. The ARO and ARI 
configurations are exactly as illustrated in Figures 9 and 10. For the SRI configuration, only the center rods are inserted into the core, as shown in Figure 12.

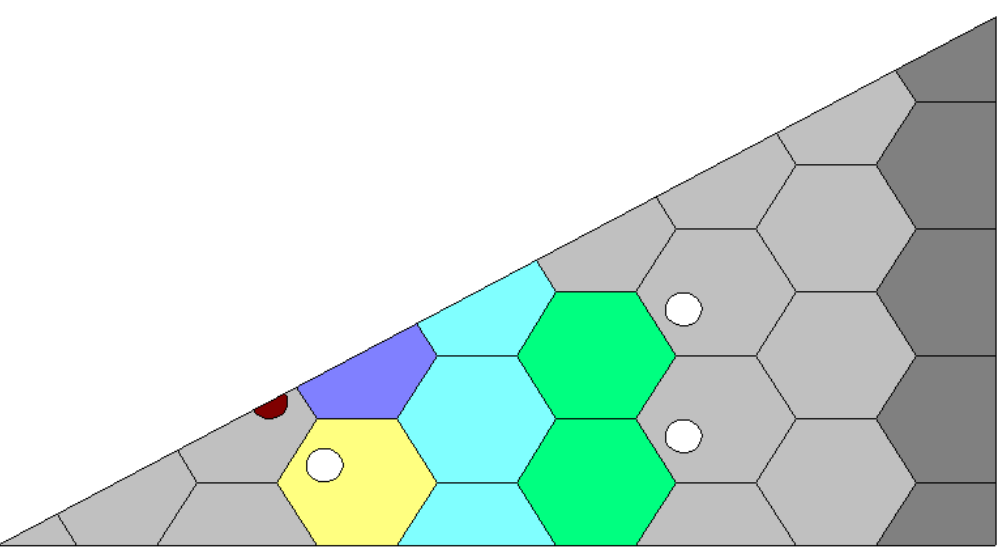

Figure 12. Block diagram for 2-D core with some rods inserted

Table 7. Block-averaged fission density values, 2-D stylized cores

\begin{tabular}{|c|c|c|c|c|c|c|c|}
\hline Block & 1 & 2 & 3 & 4 & 5 & 6 & 7 \\
\hline ARO & 1.0650 & 1.2600 & 0.9058 & 0.8584 & 0.8705 & 1.0269 & 0.9638 \\
\hline SRI & 0.8010 & 0.9360 & 0.9990 & 0.8830 & 0.8843 & 1.2100 & 1.1217 \\
\hline ARI & 1.0437 & 1.2926 & 1.0134 & 1.0178 & 1.0572 & 0.8023 & 0.8636 \\
\hline
\end{tabular}

All three configurations yielded a pin fission density profile with uncertainty averaging $0.025 \%$ for individual pin tallies, and maximum uncertainty in the pin-level data of $0.04 \%$. The eigenvalue uncertainty was found to be $2 \mathrm{pcm}$. To achieve this level of precision, 250,000 particles were simulated for 500 inactive cycles followed by 6,000 active cycles, for a total of 1.5 billion active particle histories. The block fission density results are presented in Table 7. Pin fission density figures for all three cases are provided in Tables B4-B6. A pin numbering diagram is provided in Figure 13, as 210 fuel pins are present in a standard fuel block and all are listed in each of Tables B4-B6. The numbers in the top row refer to the block, and the numbers in the left column list the pin numbers. All pin fission density figures are normalized such that the average pin in the core has a value of 1.0. 


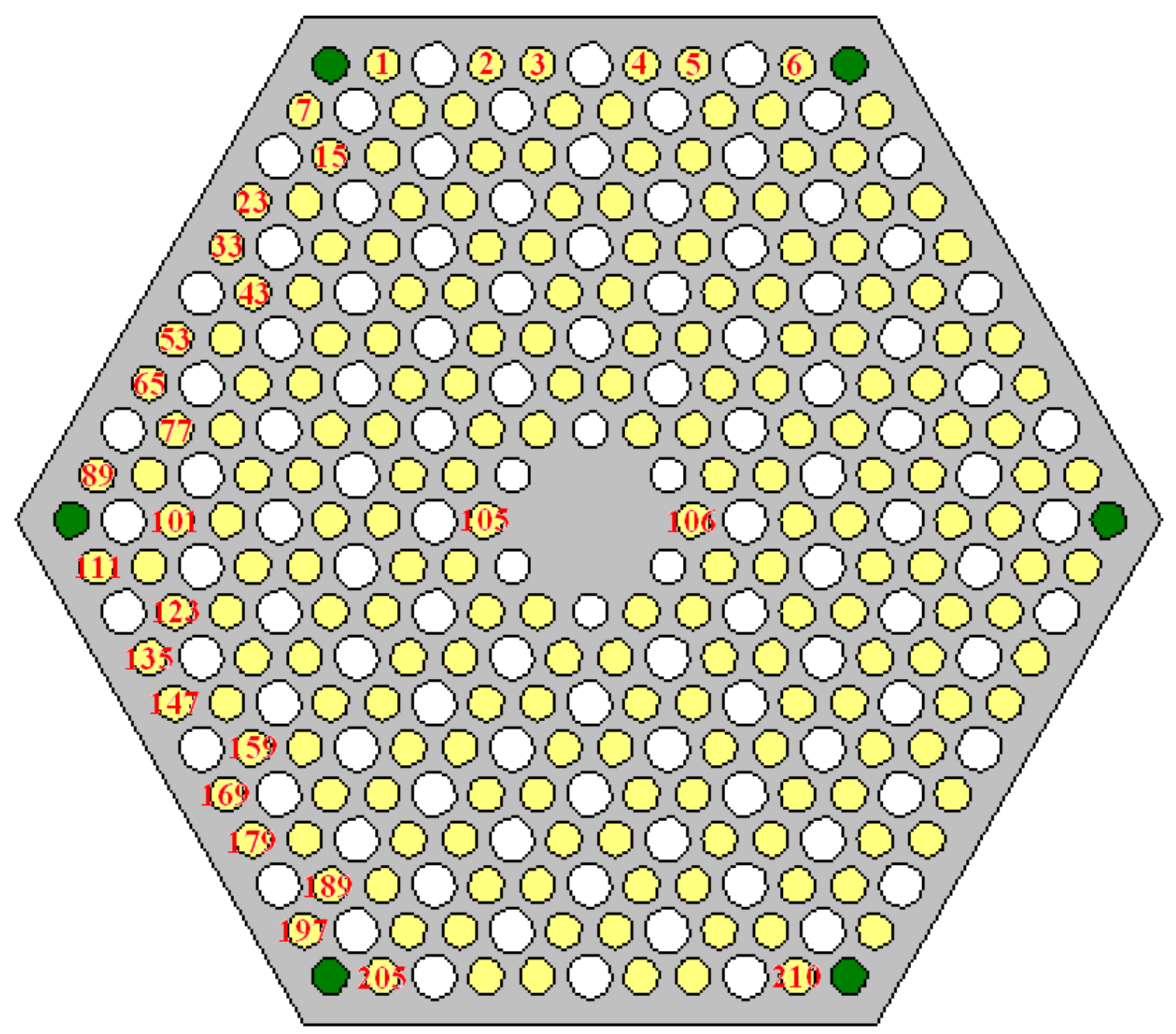

Figure 13. Pin numbering diagram for pin fission density data

It should be noted that in block 2, where there is a reserve shutdown channel, several pins are not present in the fuel block; those are blanked in the table. The same is done for pins in blocks 1,3 , and 5; where due to the core symmetry, only those pins which are within the boundaries of the slice of the core solved are provided. In block 5, where the lines of symmetry run through pins 101-110, the values given are correctly normalized to the whole block.

\section{SUMMARY AND CONCLUSIONS}

A set of benchmark problems has been presented. Two unique core configurations (all rods out and all rods in) were described for a fully heterogeneous core in continuous energy. Three configurations (all rods out, some rods in, and all rods in) were given for a 3-D multigroup stylization of the core, in which fuel and absorber pins are treated as homogeneous (flux- 
averaged), but the blocks composing the core remain highly heterogeneous. Additionally, three 2-D configurations (all rods out, center rods in, and all rods in) were presented.

It is anticipated that these benchmark problems will be used by methods developers, as the prismatic VHTR is a challenging core to model, and whole core models are currently not widely available. Potential applications for this work include use as a standard numerical verification resource for reactor core solution methods and also for cross section generation methods. A variety of issues related to cross section generation can be investigated using this benchmark, such as group structure, the number of groups necessary for a solution of desired accuracy, the necessary order to which to expand the scattering kernel, and homogenization of highly heterogeneous pins and associated complicated reactor geometry.

\section{ACKNOWLEDGEMENTS}

The first and second authors' work was supported by NEUP Award Number DE-AC07O5ID14517.

The third author's contribution involving the initial core model was supported by the U.S. Department of Energy award number DE-PS07-08ID14907.

The authors are grateful to Tom Lewis and Matthew Johnson, who contributed to the development of the initial core model.

\section{REFERENCES}

Gougar, H. et al., "Prismatic Coupled Neutronics/Thermal Fluids Transient Benchmark of the MHTGR-350 MW Core Design: Benchmark Definition,” Idaho National Laboratory (2010).

Ilas, G., “A Monte Carlo Based Nodal Diffusion Model for Criticality Analysis and Application of High-Order Cross Section Homogenization Method to Two-Group Nodal Diffusion,” Ph.D. Dissertation, Georgia Institute of Technology (2002).

MacDonald, P. E., et al., "NGNP Preliminary Point Design - Results of the Initial Neutronics and Thermal-Hydraulic Assessments,” Idaho National Engineering and Environmental Laboratory, INEEL/EXT-03-00870 (2003).

McConn, R. J., Jr., Gesh, C. J., Pagh, R. T., Rucker, R. A., Williams, R. G., III, “Compendium of Material Composition Data for Radiation Transport Modeling,” Pacific Northwest National Laboratory, PNNL-15870 Rev. 1 (2011).

Murata, I., Yamashita, K., Maruyama, S., et al., 1994. Evaluation of Local Power Distribution with Fine-mesh Core Model for High Temperature Engineering Test Reactor (HTTR). Journal of Nuclear Science and Technology. 31(1), 62-72.

Pounders, J., Rahnema, F., Connolly, K. J., 2014. The History-Partitioning Method for Multigroup Stochastic Cross Section Generation. To be submitted.

Pounders, J., Rahnema, F., Stamm’ler, R. J. J., "Stochastically Generated Multigroup Cross Sections," The First International Conference on Physics and Technology of Reactors and Applications, Marrakech, Morocco, March 14-17 (2007). 
Williams, P. M., King, T. L., Wilson, J. N., “Draft Preapplication Safety Evaluation Report for the Modular High-Temperature Gas-Cooled Reactor”, U.S. Nuclear Regulatory Commission, NUREG-1338 (1989).

X-5 Monte Carlo Team, 2005. MCNP-A General Monte Carlo N-Particle Transport Code, Version 5. Los Alamos National Laboratory.

Yang, X., Satvat, N., 2012. MOCUM: A two-dimensional method of characteristics code based on constructive solid geometry and unstructured meshing for general geometries. Annals of Nuclear Energy. 46, 20-28.

Zhang, Z., Rahnema, F., Zhang, D., Pounders, J. M., Ougouag, A., 2011. Simplified Two and Three Dimensional HTTR Benchmark Problems. Annals of Nuclear Energy. 38, 1172-85.

\section{APPENDIX A: CROSS SECTION DATA}

Cross section data is given for all materials in units of $\mathrm{cm}^{-1}$. Data is presented as group number followed by the macroscopic cross section, or, in the case of the fission spectrum, as the quantity of fission neutrons per group per fission neutron. The scattering matrix is also presented for P0 and P1 scattering.

The colors used to illustrate the material or block in the various figures presented in the paper is given in parentheses for clarification.

Inner fuel (fuel in yellow blocks)

\begin{tabular}{|c|c|}
\hline \multicolumn{2}{|c|}{ CAPTURE CROSS SECTION } \\
\hline 1 & 1.29633E-04 \\
\hline 2 & 7.84585E-04 \\
\hline 3 & 1.22677E-02 \\
\hline 4 & $1.98430 \mathrm{E}-03$ \\
\hline 5 & 5.80629E-03 \\
\hline 6 & $1.08130 \mathrm{E}-02$ \\
\hline \multicolumn{2}{|c|}{ FISSION CROSS SECTION } \\
\hline 1 & 3.30605E-04 \\
\hline 2 & 4.94507E-04 \\
\hline 3 & 4.18507E-03 \\
\hline 4 & $6.24056 \mathrm{E}-03$ \\
\hline 5 & $2.48268 \mathrm{E}-02$ \\
\hline 6 & $5.39230 \mathrm{E}-02$ \\
\hline \multicolumn{2}{|c|}{ NU-FISSION CROSS SECTION } \\
\hline 1 & 8.75978E-04 \\
\hline 2 & 1.20327E-03 \\
\hline 3 & 1.01927E-02 \\
\hline 4 & 1.52064E-02 \\
\hline 5 & 6.04956E-02 \\
\hline 6 & 1.31394E-01 \\
\hline \multicolumn{2}{|c|}{ FISSION SPECTRUM } \\
\hline & 10.969367608193630 \\
\hline & 2 3.061677199212420E-002 \\
\hline & 3 1.561981424628129E-005 \\
\hline
\end{tabular}



$4 \quad 0.000000000000000 \mathrm{E}+000$
$50.000000000000000 \mathrm{E}+000$
$\begin{array}{ll}6 & 0.000000000000000 \mathrm{E}+000\end{array}$

$\begin{array}{cccccc}\text { P0 SCATTERING: } & & & & \\ 1.60326 \mathrm{E}-01 & 1.16923 \mathrm{E}-02 & 2.21020 \mathrm{E}-08 & 0.00000 \mathrm{E}+00 & 0.00000 \mathrm{E}+00 & 0.00000 \mathrm{E}+00 \\ 0.00000 \mathrm{E}+00 & 2.62149 \mathrm{E}-01 & 7.94082 \mathrm{E}-03 & 0.00000 \mathrm{E}+00 & 0.00000 \mathrm{E}+00 & 0.00000 \mathrm{E}+00 \\ 0.00000 \mathrm{E}+00 & 0.00000 \mathrm{E}+00 & 2.75777 \mathrm{E}-01 & 5.94908 \mathrm{E}-03 & 0.00000 \mathrm{E}+00 & 0.00000 \mathrm{E}+00 \\ 0.00000 \mathrm{E}+00 & 0.00000 \mathrm{E}+00 & 5.84465 \mathrm{E}-04 & 2.37923 \mathrm{E}-01 & 3.58179 \mathrm{E}-02 & 0.00000 \mathrm{E}+00 \\ 0.00000 \mathrm{E}+00 & 0.00000 \mathrm{E}+00 & 0.00000 \mathrm{E}+00 & 9.27362 \mathrm{E}-04 & 2.45405 \mathrm{E}-01 & 3.15433 \mathrm{E}-02 \\ 0.00000 \mathrm{E}+00 & 0.00000 \mathrm{E}+00 & 0.00000 \mathrm{E}+00 & 0.00000 \mathrm{E}+00 & 7.87841 \mathrm{E}-02 & 2.11178 \mathrm{E}-01 \\ \text { P1 SCATTERING: } & & & & \\ 2.10489 \mathrm{E}-02 & -3.24888 \mathrm{E}-03 & 7.66141 \mathrm{E}-09 & 0.00000 \mathrm{E}+00 & 0.00000 \mathrm{E}+00 & 0.00000 \mathrm{E}+00 \\ 0.00000 \mathrm{E}+00 & 1.85639 \mathrm{E}-02 & -2.34063 \mathrm{E}-03 & 0.00000 \mathrm{E}+00 & 0.00000 \mathrm{E}+00 & 0.00000 \mathrm{E}+00 \\ 0.00000 \mathrm{E}+00 & 0.00000 \mathrm{E}+00 & 1.66272 \mathrm{E}-02 & -1.68169 \mathrm{E}-03 & 0.00000 \mathrm{E}+00 & 0.00000 \mathrm{E}+00 \\ 0.00000 \mathrm{E}+00 & 0.00000 \mathrm{E}+00 & 1.93229 \mathrm{E}-04 & 1.13352 \mathrm{E}-02 & -8.93634 \mathrm{E}-03 & 0.00000 \mathrm{E}+00 \\ 0.00000 \mathrm{E}+00 & 0.00000 \mathrm{E}+00 & 0.00000 \mathrm{E}+00 & -7.72176 \mathrm{E}-05 & -2.96860 \mathrm{E}-02 & -7.58348 \mathrm{E}-03 \\ 0.00000 \mathrm{E}+00 & 0.00000 \mathrm{E}+00 & 0.00000 \mathrm{E}+00 & 0.00000 \mathrm{E}+00 & -1.87819 \mathrm{E}-02 & -4.98282 \mathrm{E}-02\end{array}$

Middle fuel (fuel in aqua blocks)

\footnotetext{
FISSION CROSS SECTION

1 3.23025E-04

2 4.99872E-04

3 4.17914E-03

$4 \quad 6.21066 \mathrm{E}-03$

$5 \quad 2.38835 \mathrm{E}-02$

$6 \quad 5.26801 \mathrm{E}-02$
}

\section{FISSION SPECTRUM}
10.969314801101188
2 3.067047740275138E-002
3 1.469671239748238E-005
$40.000000000000000 \mathrm{E}+000$
5 2.478366340216252E-008
$60.000000000000000 \mathrm{E}+000$

PO SCATTERING:

$\begin{array}{cccccc}1.61605 \mathrm{E}-01 & 1.17793 \mathrm{E}-02 & 5.60222 \mathrm{E}-08 & 0.00000 \mathrm{E}+00 & 0.00000 \mathrm{E}+00 & 0.00000 \mathrm{E}+00 \\ 0.00000 \mathrm{E}+00 & 2.62338 \mathrm{E}-01 & 7.94475 \mathrm{E}-03 & 0.00000 \mathrm{E}+00 & 0.00000 \mathrm{E}+00 & 0.00000 \mathrm{E}+00\end{array}$




$\begin{array}{rlllll}0.00000 \mathrm{E}+00 & 0.00000 \mathrm{E}+00 & 2.75875 \mathrm{E}-01 & 5.95029 \mathrm{E}-03 & 0.00000 \mathrm{E}+00 & 0.00000 \mathrm{E}+00 \\ 0.00000 \mathrm{E}+00 & 0.00000 \mathrm{E}+00 & 5.88892 \mathrm{E}-04 & 2.37946 \mathrm{E}-01 & 3.58974 \mathrm{E}-02 & 0.00000 \mathrm{E}+00 \\ 0.00000 \mathrm{E}+00 & 0.00000 \mathrm{E}+00 & 0.00000 \mathrm{E}+00 & 9.26591 \mathrm{E}-04 & 2.45647 \mathrm{E}-01 & 3.15620 \mathrm{E}-02 \\ 0.00000 \mathrm{E}+00 & 0.00000 \mathrm{E}+00 & 0.00000 \mathrm{E}+00 & 0.00000 \mathrm{E}+00 & 7.91191 \mathrm{E}-02 & 2.12103 \mathrm{E}-01 \\ \text { P1 SCATTERING: } & & & & & \\ 2.12413 \mathrm{E}-02 & -3.26818 \mathrm{E}-03 & 3.25127 \mathrm{E}-08 & 0.00000 \mathrm{E}+00 & 0.00000 \mathrm{E}+00 & 0.00000 \mathrm{E}+00 \\ 0.00000 \mathrm{E}+00 & 1.86016 \mathrm{E}-02 & -2.34678 \mathrm{E}-03 & 0.00000 \mathrm{E}+00 & 0.00000 \mathrm{E}+00 & 0.00000 \mathrm{E}+00 \\ 0.00000 \mathrm{E}+00 & 0.00000 \mathrm{E}+00 & 1.66653 \mathrm{E}-02 & -1.67778 \mathrm{E}-03 & 0.00000 \mathrm{E}+00 & 0.00000 \mathrm{E}+00 \\ 0.00000 \mathrm{E}+00 & 0.00000 \mathrm{E}+00 & 1.96356 \mathrm{E}-04 & 1.13536 \mathrm{E}-02 & -8.91061 \mathrm{E}-03 & 0.00000 \mathrm{E}+00 \\ 0.00000 \mathrm{E}+00 & 0.00000 \mathrm{E}+00 & 0.00000 \mathrm{E}+00 & -7.72556 \mathrm{E}-05 & -2.97309 \mathrm{E}-02 & -7.60206 \mathrm{E}-03 \\ 0.00000 \mathrm{E}+00 & 0.00000 \mathrm{E}+00 & 0.00000 \mathrm{E}+00 & 0.00000 \mathrm{E}+00 & -1.88686 \mathrm{E}-02 & -5.00512 \mathrm{E}-02\end{array}$

Outer fuel (fuel in green blocks)

$$
\begin{array}{cc}
\multicolumn{2}{c}{\text { CAPTURE CROSS SECTION }} \\
1 & 1.28370 \mathrm{E}-04 \\
2 & 7.78002 \mathrm{E}-04 \\
3 & 1.21394 \mathrm{E}-02 \\
4 & 1.98183 \mathrm{E}-03 \\
5 & 5.83706 \mathrm{E}-03 \\
6 & 1.08275 \mathrm{E}-02
\end{array}
$$

\section{FISSION CROSS SECTION}

$\begin{array}{cc}1 & 3.32508 \mathrm{E}-04 \\ 2 & 4.92273 \mathrm{E}-04 \\ 3 & 4.18350 \mathrm{E}-03 \\ 4 & 6.25056 \mathrm{E}-03 \\ 5 & 2.49620 \mathrm{E}-02 \\ 6 & 5.39956 \mathrm{E}-02\end{array}$

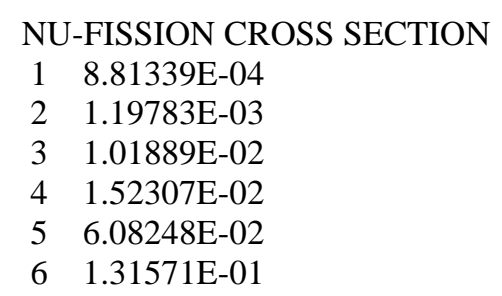

\section{FISSION SPECTRUM}
10.969360837387438
2 3.062414611195433E-002
3 1.501650060802793E-005
$40.000000000000000 \mathrm{E}+000$
$5 \quad 0.000000000000000 \mathrm{E}+000$
6
$0.000000000000000 \mathrm{E}+000$

P0 SCATTERING:

$\begin{array}{rccccc}1.59884 \mathrm{E}-01 & 1.16471 \mathrm{E}-02 & 0.00000 \mathrm{E}+00 & 0.00000 \mathrm{E}+00 & 0.00000 \mathrm{E}+00 & 0.00000 \mathrm{E}+00 \\ 0.00000 \mathrm{E}+00 & 2.61990 \mathrm{E}-01 & 7.93533 \mathrm{E}-03 & 0.00000 \mathrm{E}+00 & 0.00000 \mathrm{E}+00 & 0.00000 \mathrm{E}+00 \\ 0.00000 \mathrm{E}+00 & 0.00000 \mathrm{E}+00 & 2.75812 \mathrm{E}-01 & 5.94570 \mathrm{E}-03 & 0.00000 \mathrm{E}+00 & 0.00000 \mathrm{E}+00 \\ 0.00000 \mathrm{E}+00 & 0.00000 \mathrm{E}+00 & 5.85318 \mathrm{E}-04 & 2.37895 \mathrm{E}-01 & 3.59055 \mathrm{E}-02 & 0.00000 \mathrm{E}+00 \\ 0.00000 \mathrm{E}+00 & 0.00000 \mathrm{E}+00 & 0.00000 \mathrm{E}+00 & 9.32976 \mathrm{E}-04 & 2.45296 \mathrm{E}-01 & 3.15157 \mathrm{E}-02 \\ 0.00000 \mathrm{E}+00 & 0.00000 \mathrm{E}+00 & 0.00000 \mathrm{E}+00 & 0.00000 \mathrm{E}+00 & 7.87611 \mathrm{E}-02 & 2.11125 \mathrm{E}-01 \\ \text { P1 SCATTERING: } & & & & & \\ 2.09414 \mathrm{E}-02 & -3.23034 \mathrm{E}-03 & 0.00000 \mathrm{E}+00 & 0.00000 \mathrm{E}+00 & 0.00000 \mathrm{E}+00 & 0.00000 \mathrm{E}+00\end{array}$




$\begin{array}{cccccc}0.00000 \mathrm{E}+00 & 1.85611 \mathrm{E}-02 & -2.34093 \mathrm{E}-03 & 0.00000 \mathrm{E}+00 & 0.00000 \mathrm{E}+00 & 0.00000 \mathrm{E}+00 \\ 0.00000 \mathrm{E}+00 & 0.00000 \mathrm{E}+00 & 1.66766 \mathrm{E}-02 & -1.67325 \mathrm{E}-03 & 0.00000 \mathrm{E}+00 & 0.00000 \mathrm{E}+00 \\ 0.00000 \mathrm{E}+00 & 0.00000 \mathrm{E}+00 & 1.97655 \mathrm{E}-04 & 1.14205 \mathrm{E}-02 & -8.94440 \mathrm{E}-03 & 0.00000 \mathrm{E}+00 \\ 0.00000 \mathrm{E}+00 & 0.00000 \mathrm{E}+00 & 0.00000 \mathrm{E}+00 & -7.81716 \mathrm{E}-05 & -2.96594 \mathrm{E}-02 & -7.58906 \mathrm{E}-03 \\ 0.00000 \mathrm{E}+00 & 0.00000 \mathrm{E}+00 & 0.00000 \mathrm{E}+00 & 0.00000 \mathrm{E}+00 & -1.88138 \mathrm{E}-02 & -4.97992 \mathrm{E}-02\end{array}$

Burnable absorber/poison pins (dark green material in all fuel blocks)

\section{3.93318E-04 \\ $2 \quad 4.01561 \mathrm{E}-03$ \\ $3 \quad 6.06652 \mathrm{E}-02$ \\ $4 \quad 2.95121 \mathrm{E}-01$ \\ $5 \quad 5.61362 \mathrm{E}-01$ \\ $6 \quad 8.46069 \mathrm{E}-01$}

CAPTURE CROSS SECTION

\section{P0 SCATTERING:}

$\begin{array}{cccccc}1.22600 \mathrm{E}-01 & 8.94531 \mathrm{E}-03 & 3.49147 \mathrm{E}-08 & 0.00000 \mathrm{E}+00 & 0.00000 \mathrm{E}+00 & 0.00000 \mathrm{E}+00 \\ 0.00000 \mathrm{E}+00 & 2.07325 \mathrm{E}-01 & 6.27909 \mathrm{E}-03 & 0.00000 \mathrm{E}+00 & 0.00000 \mathrm{E}+00 & 0.00000 \mathrm{E}+00 \\ 0.00000 \mathrm{E}+00 & 0.00000 \mathrm{E}+00 & 2.16128 \mathrm{E}-01 & 4.66294 \mathrm{E}-03 & 0.00000 \mathrm{E}+00 & 0.00000 \mathrm{E}+00 \\ 0.00000 \mathrm{E}+00 & 0.00000 \mathrm{E}+00 & 4.87089 \mathrm{E}-04 & 1.93308 \mathrm{E}-01 & 2.91572 \mathrm{E}-02 & 0.00000 \mathrm{E}+00 \\ 0.00000 \mathrm{E}+00 & 0.00000 \mathrm{E}+00 & 0.00000 \mathrm{E}+00 & 7.66395 \mathrm{E}-04 & 2.02635 \mathrm{E}-01 & 2.60543 \mathrm{E}-02 \\ 0.00000 \mathrm{E}+00 & 0.00000 \mathrm{E}+00 & 0.00000 \mathrm{E}+00 & 0.00000 \mathrm{E}+00 & 6.61547 \mathrm{E}-02 & 1.77300 \mathrm{E}-01 \\ \text { P1 SCATTERING: } & & & & \\ 1.60769 \mathrm{E}-02 & -2.47491 \mathrm{E}-03 & 5.04902 \mathrm{E}-09 & 0.00000 \mathrm{E}+00 & 0.00000 \mathrm{E}+00 & 0.00000 \mathrm{E}+00 \\ 0.00000 \mathrm{E}+00 & 1.47206 \mathrm{E}-02 & -1.85069 \mathrm{E}-03 & 0.00000 \mathrm{E}+00 & 0.00000 \mathrm{E}+00 & 0.00000 \mathrm{E}+00 \\ 0.00000 \mathrm{E}+00 & 0.00000 \mathrm{E}+00 & 1.30263 \mathrm{E}-02 & -1.31769 \mathrm{E}-03 & 0.00000 \mathrm{E}+00 & 0.00000 \mathrm{E}+00 \\ 0.00000 \mathrm{E}+00 & 0.00000 \mathrm{E}+00 & 1.63363 \mathrm{E}-04 & 9.17567 \mathrm{E}-03 & -7.24006 \mathrm{E}-03 & 0.00000 \mathrm{E}+00 \\ 0.00000 \mathrm{E}+00 & 0.00000 \mathrm{E}+00 & 0.00000 \mathrm{E}+00 & -6.43132 \mathrm{E}-05 & -2.44902 \mathrm{E}-02 & -6.27652 \mathrm{E}-03 \\ 0.00000 \mathrm{E}+00 & 0.00000 \mathrm{E}+00 & 0.00000 \mathrm{E}+00 & 0.00000 \mathrm{E}+00 & -1.57961 \mathrm{E}-02 & -4.17978 \mathrm{E}-02\end{array}$

Inner fuel block graphite (graphite in yellow blocks)

$$
\begin{array}{ll}
1 & 2.56623 \mathrm{E}-05 \\
2 & 6.20603 \mathrm{E}-07 \\
3 & 1.07303 \mathrm{E}-05 \\
4 & 5.51553 \mathrm{E}-05 \\
5 & 1.28799 \mathrm{E}-04 \\
6 & 2.63572 \mathrm{E}-04
\end{array}
$$$$
\text { CAPTURE CROSS SECTION }
$$

P0 SCATTERING:

$\begin{array}{lcccccc}2.28798 \mathrm{E}-01 & 1.66939 \mathrm{E}-02 & 6.51582 \mathrm{E}-08 & 0.00000 \mathrm{E}+00 & 0.00000 \mathrm{E}+00 & 0.00000 \mathrm{E}+00 \\ 0.00000 \mathrm{E}+00 & 3.90649 \mathrm{E}-01 & 1.18313 \mathrm{E}-02 & 0.00000 \mathrm{E}+00 & 0.00000 \mathrm{E}+00 & 0.00000 \mathrm{E}+00 \\ 0.00000 \mathrm{E}+00 & 0.00000 \mathrm{E}+00 & 4.04628 \mathrm{E}-01 & 8.72981 \mathrm{E}-03 & 0.00000 \mathrm{E}+00 & 0.00000 \mathrm{E}+00 \\ 0.00000 \mathrm{E}+00 & 0.00000 \mathrm{E}+00 & 9.04253 \mathrm{E}-04 & 3.58866 \mathrm{E}-01 & 5.41286 \mathrm{E}-02 & 0.00000 \mathrm{E}+00 \\ 0.00000 \mathrm{E}+00 & 0.00000 \mathrm{E}+00 & 0.00000 \mathrm{E}+00 & 1.39103 \mathrm{E}-03 & 3.67789 \mathrm{E}-01 & 4.72892 \mathrm{E}-02 \\ 0.00000 \mathrm{E}+00 & 0.00000 \mathrm{E}+00 & 0.00000 \mathrm{E}+00 & 0.00000 \mathrm{E}+00 & 1.17813 \mathrm{E}-01 & 3.15748 \mathrm{E}-01 \\ \text { P1 SCATTERING: } & & & & & \\ \text { 3.00030E-02 } & -4.61872 \mathrm{E}-03 & 9.42255 \mathrm{E}-09 & 0.00000 \mathrm{E}+00 & 0.00000 \mathrm{E}+00 & 0.00000 \mathrm{E}+00 \\ 0.00000 \mathrm{E}+00 & 2.77371 \mathrm{E}-02 & -3.48715 \mathrm{E}-03 & 0.00000 \mathrm{E}+00 & 0.00000 \mathrm{E}+00 & 0.00000 \mathrm{E}+00 \\ 0.00000 \mathrm{E}+00 & 0.00000 \mathrm{E}+00 & 2.43875 \mathrm{E}-02 & -2.46694 \mathrm{E}-03 & 0.00000 \mathrm{E}+00 & 0.00000 \mathrm{E}+00 \\ 0.00000 \mathrm{E}+00 & 0.00000 \mathrm{E}+00 & 3.03275 \mathrm{E}-04 & 1.70341 \mathrm{E}-02 & -1.34408 \mathrm{E}-02 & 0.00000 \mathrm{E}+00 \\ 0.00000 \mathrm{E}+00 & 0.00000 \mathrm{E}+00 & 0.00000 \mathrm{E}+00 & -1.16730 \mathrm{E}-04 & -4.44505 \mathrm{E}-02 & -1.13921 \mathrm{E}-02 \\ 0.00000 \mathrm{E}+00 & 0.00000 \mathrm{E}+00 & 0.00000 \mathrm{E}+00 & 0.00000 \mathrm{E}+00 & -2.81308 \mathrm{E}-02 & -7.44364 \mathrm{E}-02\end{array}$


Middle fuel block graphite (graphite in aqua blocks)

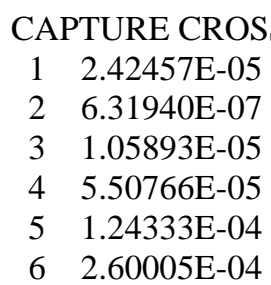

P0 SCATTERING:

$\begin{array}{cccccc}2.30275 \mathrm{E}-01 & 1.68016 \mathrm{E}-02 & 6.55788 \mathrm{E}-08 & 0.00000 \mathrm{E}+00 & 0.00000 \mathrm{E}+00 & 0.00000 \mathrm{E}+00 \\ 0.00000 \mathrm{E}+00 & 3.90891 \mathrm{E}-01 & 1.18386 \mathrm{E}-02 & 0.00000 \mathrm{E}+00 & 0.00000 \mathrm{E}+00 & 0.00000 \mathrm{E}+00 \\ 0.00000 \mathrm{E}+00 & 0.00000 \mathrm{E}+00 & 4.04628 \mathrm{E}-01 & 8.72981 \mathrm{E}-03 & 0.00000 \mathrm{E}+00 & 0.00000 \mathrm{E}+00 \\ 0.00000 \mathrm{E}+00 & 0.00000 \mathrm{E}+00 & 9.04251 \mathrm{E}-04 & 3.58865 \mathrm{E}-01 & 5.41284 \mathrm{E}-02 & 0.00000 \mathrm{E}+00 \\ 0.00000 \mathrm{E}+00 & 0.00000 \mathrm{E}+00 & 0.00000 \mathrm{E}+00 & 1.39020 \mathrm{E}-03 & 3.67569 \mathrm{E}-01 & 4.72609 \mathrm{E}-02 \\ 0.00000 \mathrm{E}+00 & 0.00000 \mathrm{E}+00 & 0.00000 \mathrm{E}+00 & 0.00000 \mathrm{E}+00 & 1.17772 \mathrm{E}-01 & 3.15640 \mathrm{E}-01 \\ \mathrm{P} 1 \mathrm{SCATTERING} & & & & & \\ 3.01967 \mathrm{E}-02 & -4.64853 \mathrm{E}-03 & 9.48336 \mathrm{E}-09 & 0.00000 \mathrm{E}+00 & 0.00000 \mathrm{E}+00 & 0.00000 \mathrm{E}+00 \\ 0.00000 \mathrm{E}+00 & 2.77542 \mathrm{E}-02 & -3.48931 \mathrm{E}-03 & 0.00000 \mathrm{E}+00 & 0.00000 \mathrm{E}+00 & 0.00000 \mathrm{E}+00 \\ 0.00000 \mathrm{E}+00 & 0.00000 \mathrm{E}+00 & 2.43875 \mathrm{E}-02 & -2.46694 \mathrm{E}-03 & 0.00000 \mathrm{E}+00 & 0.00000 \mathrm{E}+00 \\ 0.00000 \mathrm{E}+00 & 0.00000 \mathrm{E}+00 & 3.03274 \mathrm{E}-04 & 1.70340 \mathrm{E}-02 & -1.34407 \mathrm{E}-02 & 0.00000 \mathrm{E}+00 \\ 0.00000 \mathrm{E}+00 & 0.00000 \mathrm{E}+00 & 0.00000 \mathrm{E}+00 & -1.16661 \mathrm{E}-04 & -4.44239 \mathrm{E}-02 & -1.13852 \mathrm{E}-02 \\ 0.00000 \mathrm{E}+00 & 0.00000 \mathrm{E}+00 & 0.00000 \mathrm{E}+00 & 0.00000 \mathrm{E}+00 & -2.81212 \mathrm{E}-02 & -7.44108 \mathrm{E}-02\end{array}$

Outer fuel block graphite (graphite in green blocks)

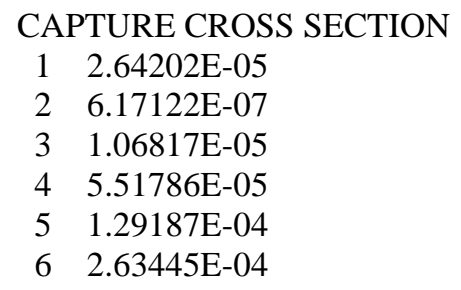

P0 SCATTERING:

$\begin{array}{cccccc}2.28373 \mathrm{E}-01 & 1.66629 \mathrm{E}-02 & 6.50372 \mathrm{E}-08 & 0.00000 \mathrm{E}+00 & 0.00000 \mathrm{E}+00 & 0.00000 \mathrm{E}+00 \\ 0.00000 \mathrm{E}+00 & 3.90572 \mathrm{E}-01 & 1.18290 \mathrm{E}-02 & 0.00000 \mathrm{E}+00 & 0.00000 \mathrm{E}+00 & 0.00000 \mathrm{E}+00 \\ 0.00000 \mathrm{E}+00 & 0.00000 \mathrm{E}+00 & 4.04627 \mathrm{E}-01 & 8.72981 \mathrm{E}-03 & 0.00000 \mathrm{E}+00 & 0.00000 \mathrm{E}+00 \\ 0.00000 \mathrm{E}+00 & 0.00000 \mathrm{E}+00 & 9.04253 \mathrm{E}-04 & 3.58866 \mathrm{E}-01 & 5.41286 \mathrm{E}-02 & 0.00000 \mathrm{E}+00 \\ 0.00000 \mathrm{E}+00 & 0.00000 \mathrm{E}+00 & 0.00000 \mathrm{E}+00 & 1.39110 \mathrm{E}-03 & 3.67807 \mathrm{E}-01 & 4.72917 \mathrm{E}-02 \\ 0.00000 \mathrm{E}+00 & 0.00000 \mathrm{E}+00 & 0.00000 \mathrm{E}+00 & 0.00000 \mathrm{E}+00 & 1.17820 \mathrm{E}-01 & 3.15766 \mathrm{E}-01 \\ \mathrm{P} 1 \mathrm{SCATTERING} & & & & & \\ 2.99473 \mathrm{E}-02 & -4.61016 \mathrm{E}-03 & 9.40505 \mathrm{E}-09 & 0.00000 \mathrm{E}+00 & 0.00000 \mathrm{E}+00 & 0.00000 \mathrm{E}+00 \\ 0.00000 \mathrm{E}+00 & 2.77315 \mathrm{E}-02 & -3.48645 \mathrm{E}-03 & 0.00000 \mathrm{E}+00 & 0.00000 \mathrm{E}+00 & 0.00000 \mathrm{E}+00 \\ 0.00000 \mathrm{E}+00 & 0.00000 \mathrm{E}+00 & 2.43875 \mathrm{E}-02 & -2.46693 \mathrm{E}-03 & 0.00000 \mathrm{E}+00 & 0.00000 \mathrm{E}+00 \\ 0.00000 \mathrm{E}+00 & 0.00000 \mathrm{E}+00 & 3.03274 \mathrm{E}-04 & 1.70341 \mathrm{E}-02 & -1.34408 \mathrm{E}-02 & 0.00000 \mathrm{E}+00 \\ 0.00000 \mathrm{E}+00 & 0.00000 \mathrm{E}+00 & 0.00000 \mathrm{E}+00 & -1.16736 \mathrm{E}-04 & -4.44527 \mathrm{E}-02 & -1.13926 \mathrm{E}-02 \\ 0.00000 \mathrm{E}+00 & 0.00000 \mathrm{E}+00 & 0.00000 \mathrm{E}+00 & 0.00000 \mathrm{E}+00 & -2.81324 \mathrm{E}-02 & -7.44406 \mathrm{E}-02\end{array}$

Reflector blocks (light gray blocks)

CAPTURE CROSS SECTION 
$1 \quad 2.00647 \mathrm{E}-05$

2 6.84947E-07

3 1.26059E-05

4 5.58632E-05

$5 \quad 1.39415 \mathrm{E}-04$

$6 \quad 2.73960 \mathrm{E}-04$

P0 SCATTERING

$\begin{array}{cccccc}2.35193 \mathrm{E}-01 & 1.71605 \mathrm{E}-02 & 6.69795 \mathrm{E}-08 & 0.00000 \mathrm{E}+00 & 0.00000 \mathrm{E}+00 & 0.00000 \mathrm{E}+00 \\ 0.00000 \mathrm{E}+00 & 3.91881 \mathrm{E}-01 & 1.18686 \mathrm{E}-02 & 0.00000 \mathrm{E}+00 & 0.00000 \mathrm{E}+00 & 0.00000 \mathrm{E}+00 \\ 0.00000 \mathrm{E}+00 & 0.00000 \mathrm{E}+00 & 4.04632 \mathrm{E}-01 & 8.72991 \mathrm{E}-03 & 0.00000 \mathrm{E}+00 & 0.00000 \mathrm{E}+00 \\ 0.00000 \mathrm{E}+00 & 0.00000 \mathrm{E}+00 & 9.04278 \mathrm{E}-04 & 3.58876 \mathrm{E}-01 & 5.41301 \mathrm{E}-02 & 0.00000 \mathrm{E}+00 \\ 0.00000 \mathrm{E}+00 & 0.00000 \mathrm{E}+00 & 0.00000 \mathrm{E}+00 & 1.39306 \mathrm{E}-03 & 3.68327 \mathrm{E}-01 & 4.73585 \mathrm{E}-02 \\ 0.00000 \mathrm{E}+00 & 0.00000 \mathrm{E}+00 & 0.00000 \mathrm{E}+00 & 0.00000 \mathrm{E}+00 & 1.17997 \mathrm{E}-01 & 3.16241 \mathrm{E}-01 \\ \text { 1 SCATTERING } & & & & & \\ 3.08417 \mathrm{E}-02 & -4.74784 \mathrm{E}-03 & 9.68593 \mathrm{E}-09 & 0.00000 \mathrm{E}+00 & 0.00000 \mathrm{E}+00 & 0.00000 \mathrm{E}+00 \\ 0.00000 \mathrm{E}+00 & 2.78245 \mathrm{E}-02 & -3.49813 \mathrm{E}-03 & 0.00000 \mathrm{E}+00 & 0.00000 \mathrm{E}+00 & 0.00000 \mathrm{E}+00 \\ 0.00000 \mathrm{E}+00 & 0.00000 \mathrm{E}+00 & 2.43878 \mathrm{E}-02 & -2.46696 \mathrm{E}-03 & 0.00000 \mathrm{E}+00 & 0.00000 \mathrm{E}+00 \\ 0.00000 \mathrm{E}+00 & 0.00000 \mathrm{E}+00 & 3.03283 \mathrm{E}-04 & 1.70345 \mathrm{E}-02 & -1.34412 \mathrm{E}-02 & 0.00000 \mathrm{E}+00 \\ 0.00000 \mathrm{E}+00 & 0.00000 \mathrm{E}+00 & 0.00000 \mathrm{E}+00 & -1.16901 \mathrm{E}-04 & -4.45155 \mathrm{E}-02 & -1.14087 \mathrm{E}-02 \\ 0.00000 \mathrm{E}+00 & 0.00000 \mathrm{E}+00 & 0.00000 \mathrm{E}+00 & 0.00000 \mathrm{E}+00 & -2.81747 \mathrm{E}-02 & -7.45524 \mathrm{E}-02\end{array}$

Hhelium coolant (white)

CAPTURE CROSS SECTION

$1 \quad 0.00000 \mathrm{E}+00$

$2 \quad 0.00000 \mathrm{E}+00$

$3 \quad 0.00000 \mathrm{E}+00$

$4 \quad 0.00000 \mathrm{E}+00$

$5 \quad 0.00000 \mathrm{E}+00$

$6 \quad 0.00000 \mathrm{E}+00$

PO SCATTERING

$\begin{array}{cccccc}8.28116 \mathrm{E}-05 & 6.04221 \mathrm{E}-06 & 2.35835 \mathrm{E}-11 & 0.00000 \mathrm{E}+00 & 0.00000 \mathrm{E}+00 & 0.00000 \mathrm{E}+00 \\ 0.00000 \mathrm{E}+00 & 1.98704 \mathrm{E}-05 & 6.01801 \mathrm{E}-07 & 0.00000 \mathrm{E}+00 & 0.00000 \mathrm{E}+00 & 0.00000 \mathrm{E}+00 \\ 0.00000 \mathrm{E}+00 & 0.00000 \mathrm{E}+00 & 1.99748 \mathrm{E}-05 & 4.30954 \mathrm{E}-07 & 0.00000 \mathrm{E}+00 & 0.00000 \mathrm{E}+00 \\ 0.00000 \mathrm{E}+00 & 0.00000 \mathrm{E}+00 & 4.46919 \mathrm{E}-08 & 1.77366 \mathrm{E}-05 & 2.67526 \mathrm{E}-06 & 0.00000 \mathrm{E}+00 \\ 0.00000 \mathrm{E}+00 & 0.00000 \mathrm{E}+00 & 0.00000 \mathrm{E}+00 & 6.92142 \mathrm{E}-08 & 1.83003 \mathrm{E}-05 & 2.35300 \mathrm{E}-06 \\ 0.00000 \mathrm{E}+00 & 0.00000 \mathrm{E}+00 & 0.00000 \mathrm{E}+00 & 0.00000 \mathrm{E}+00 & 5.93951 \mathrm{E}-06 & 1.59184 \mathrm{E}-05 \\ \text { P1 SCATTERING } & & & & & \\ 1.08594 \mathrm{E}-05 & -1.67171 \mathrm{E}-06 & 3.41041 \mathrm{E}-12 & 0.00000 \mathrm{E}+00 & 0.00000 \mathrm{E}+00 & 0.00000 \mathrm{E}+00 \\ 0.00000 \mathrm{E}+00 & 1.41085 \mathrm{E}-06 & -1.77374 \mathrm{E}-07 & 0.00000 \mathrm{E}+00 & 0.00000 \mathrm{E}+00 & 0.00000 \mathrm{E}+00 \\ 0.00000 \mathrm{E}+00 & 0.00000 \mathrm{E}+00 & 1.20391 \mathrm{E}-06 & -1.21782 \mathrm{E}-07 & 0.00000 \mathrm{E}+00 & 0.00000 \mathrm{E}+00 \\ 0.00000 \mathrm{E}+00 & 0.00000 \mathrm{E}+00 & 1.49891 \mathrm{E}-08 & 8.41894 \mathrm{E}-07 & -6.64300 \mathrm{E}-07 & 0.00000 \mathrm{E}+00 \\ 0.00000 \mathrm{E}+00 & 0.00000 \mathrm{E}+00 & 0.00000 \mathrm{E}+00 & -5.80821 \mathrm{E}-09 & -2.21175 \mathrm{E}-06 & -5.66841 \mathrm{E}-07 \\ 0.00000 \mathrm{E}+00 & 0.00000 \mathrm{E}+00 & 0.00000 \mathrm{E}+00 & 0.00000 \mathrm{E}+00 & -1.41821 \mathrm{E}-06 & -3.75269 \mathrm{E}-06\end{array}$

Control rod material (rust-colored circles) 
$6 \quad$ 6.49513E-01

\begin{tabular}{|c|c|c|c|c|c|}
\hline \multicolumn{6}{|c|}{ PO SCATTERING } \\
\hline 2.10887E-01 & $1.53620 \mathrm{E}-02$ & 1.59075E-08 & $0.00000 \mathrm{E}+00$ & $0.00000 \mathrm{E}+00$ & $0.00000 \mathrm{E}+00$ \\
\hline $0.00000 \mathrm{E}+00$ & 4.26659E-01 & 1.28697E-02 & $0.00000 \mathrm{E}+00$ & $0.00000 \mathrm{E}+00$ & $0.00000 \mathrm{E}+00$ \\
\hline $0.00000 \mathrm{E}+00$ & $0.00000 \mathrm{E}+00$ & $9.12941 \mathrm{E}-01$ & 1.96927E-02 & $0.00000 \mathrm{E}+00$ & $0.00000 \mathrm{E}+00$ \\
\hline $0.00000 \mathrm{E}+00$ & $0.00000 \mathrm{E}+00$ & 2.46992E-03 & $9.87545 \mathrm{E}-01$ & 1.49592E-01 & $0.00000 \mathrm{E}+00$ \\
\hline $0.00000 \mathrm{E}+00$ & $0.00000 \mathrm{E}+00$ & $0.00000 \mathrm{E}+00$ & 3.57363E-03 & $1.02555 \mathrm{E}+00$ & 1.33564E-01 \\
\hline $0.00000 \mathrm{E}+00$ & $0.00000 \mathrm{E}+00$ & $0.00000 \mathrm{E}+00$ & $0.00000 \mathrm{E}+00$ & 3.18910E-01 & 2E-01 \\
\hline \multicolumn{6}{|c|}{ P1 SCATTERING } \\
\hline 2.76837E-02 & $-4.25631 \mathrm{E}-03$ & 5.65739E-09 & $0.00000 \mathrm{E}+00$ & $0.00000 \mathrm{E}+00$ & $0.00000 \mathrm{E}+00$ \\
\hline $0.00000 \mathrm{E}+00$ & 3.02101E-02 & -3.79549E-03 & $0.00000 \mathrm{E}+00$ & $0.00000 \mathrm{E}+00$ & $0.00000 \mathrm{E}+00$ \\
\hline $0.00000 \mathrm{E}+00$ & $0.00000 \mathrm{E}+00$ & 5.50224E-02 & -5.56723E-03 & $0.00000 \mathrm{E}+00$ & $0.00000 \mathrm{E}+00$ \\
\hline $0.00000 \mathrm{E}+00$ & $0.00000 \mathrm{E}+00$ & $8.19000 \mathrm{E}-04$ & 4.71136E-02 & -3.71382E-02 & $0.00000 \mathrm{E}+00$ \\
\hline+00 & $0.00000 \mathrm{E}+00$ & $0.00000 \mathrm{E}+00$ & -3.07292E-04 & $-1.24835 E-01$ & $-3.21426 \mathrm{E}-02$ \\
\hline $0.00000 \mathrm{E}+00$ & $0.00000 \mathrm{E}+00$ & $0.00000 \mathrm{E}+00$ & $0.00000 \mathrm{E}+00$ & $-7.60590 \mathrm{E}-02$ & $-2.02085 E-01$ \\
\hline
\end{tabular}

Ccontrol block graphite (gray, but in blocks with rust-colored circle)

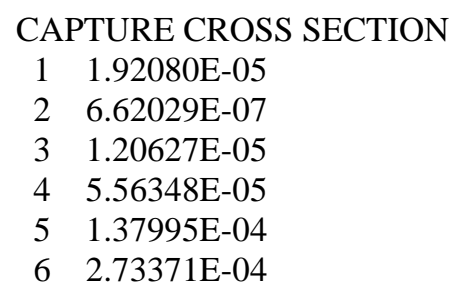

$\begin{array}{llllcc}\text { P0 SCATTERING } & & & \\ 2.36710 \mathrm{E}-01 & 1.72430 \mathrm{E}-02 & 1.78553 \mathrm{E}-08 & 0.00000 \mathrm{E}+00 & 0.00000 \mathrm{E}+00 & 0.00000 \mathrm{E}+00 \\ 0.00000 \mathrm{E}+00 & 3.91605 \mathrm{E}-01 & 1.18124 \mathrm{E}-02 & 0.00000 \mathrm{E}+00 & 0.00000 \mathrm{E}+00 & 0.00000 \mathrm{E}+00 \\ 0.00000 \mathrm{E}+00 & 0.00000 \mathrm{E}+00 & 4.04632 \mathrm{E}-01 & 8.72812 \mathrm{E}-03 & 0.00000 \mathrm{E}+00 & 0.00000 \mathrm{E}+00 \\ 0.00000 \mathrm{E}+00 & 0.00000 \mathrm{E}+00 & 8.97079 \mathrm{E}-04 & 3.58678 \mathrm{E}-01 & 5.43320 \mathrm{E}-02 & 0.00000 \mathrm{E}+00 \\ 0.00000 \mathrm{E}+00 & 0.00000 \mathrm{E}+00 & 0.00000 \mathrm{E}+00 & 1.28170 \mathrm{E}-03 & 3.67818 \mathrm{E}-01 & 4.79032 \mathrm{E}-02 \\ 0.00000 \mathrm{E}+00 & 0.00000 \mathrm{E}+00 & 0.00000 \mathrm{E}+00 & 0.00000 \mathrm{E}+00 & 1.17823 \mathrm{E}-01 & 3.16336 \mathrm{E}-01 \\ \text { P1 SCATTERING } & & & & & \\ 3.10735 \mathrm{E}-02 & -4.77748 \mathrm{E}-03 & 6.35012 \mathrm{E}-09 & 0.00000 \mathrm{E}+00 & 0.00000 \mathrm{E}+00 & 0.00000 \mathrm{E}+00 \\ 0.00000 \mathrm{E}+00 & 2.77280 \mathrm{E}-02 & -3.48366 \mathrm{E}-03 & 0.00000 \mathrm{E}+00 & 0.00000 \mathrm{E}+00 & 0.00000 \mathrm{E}+00 \\ 0.00000 \mathrm{E}+00 & 0.00000 \mathrm{E}+00 & 2.43869 \mathrm{E}-02 & -2.46749 \mathrm{E}-03 & 0.00000 \mathrm{E}+00 & 0.00000 \mathrm{E}+00 \\ 0.00000 \mathrm{E}+00 & 0.00000 \mathrm{E}+00 & 2.97462 \mathrm{E}-04 & 1.71117 \mathrm{E}-02 & -1.34886 \mathrm{E}-02 & 0.00000 \mathrm{E}+00 \\ 0.00000 \mathrm{E}+00 & 0.00000 \mathrm{E}+00 & 0.00000 \mathrm{E}+00 & -1.10212 \mathrm{E}-04 & -4.47726 \mathrm{E}-02 & -1.15281 \mathrm{E}-02 \\ 0.00000 \mathrm{E}+00 & 0.00000 \mathrm{E}+00 & 0.00000 \mathrm{E}+00 & 0.00000 \mathrm{E}+00 & -2.81004 \mathrm{E}-02 & -7.46612 \mathrm{E}-02\end{array}$

Fuel in blocks adjacent to control rods (lavender blocks)

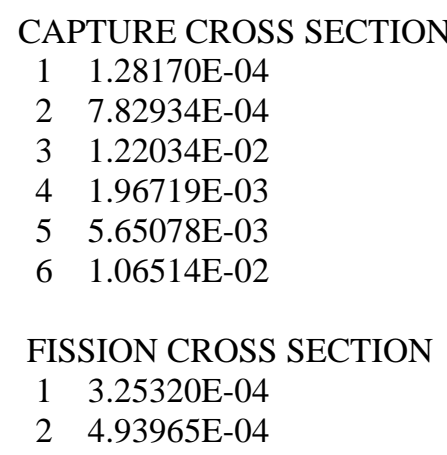




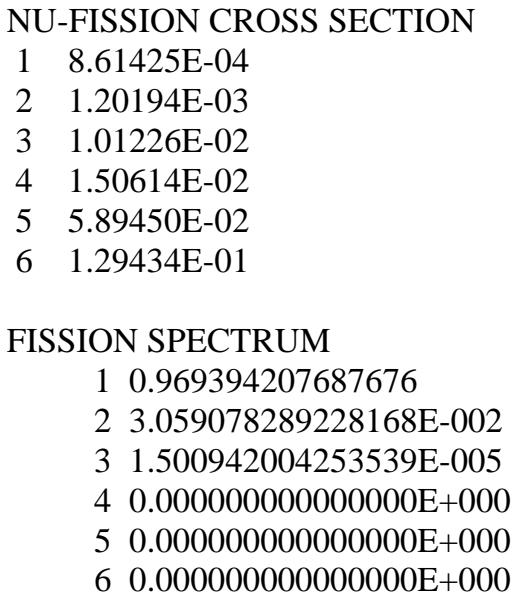

PO SCATTERING

$\begin{array}{cccccc}1.61333 \mathrm{E}-01 & 1.17633 \mathrm{E}-02 & 2.43156 \mathrm{E}-08 & 0.00000 \mathrm{E}+00 & 0.00000 \mathrm{E}+00 & 0.00000 \mathrm{E}+00 \\ 0.00000 \mathrm{E}+00 & 2.63137 \mathrm{E}-01 & 7.93873 \mathrm{E}-03 & 0.00000 \mathrm{E}+00 & 0.00000 \mathrm{E}+00 & 0.00000 \mathrm{E}+00 \\ 0.00000 \mathrm{E}+00 & 0.00000 \mathrm{E}+00 & 2.76756 \mathrm{E}-01 & 5.96534 \mathrm{E}-03 & 0.00000 \mathrm{E}+00 & 0.00000 \mathrm{E}+00 \\ 0.00000 \mathrm{E}+00 & 0.00000 \mathrm{E}+00 & 5.91983 \mathrm{E}-04 & 2.38495 \mathrm{E}-01 & 3.61417 \mathrm{E}-02 & 0.00000 \mathrm{E}+00 \\ 0.00000 \mathrm{E}+00 & 0.00000 \mathrm{E}+00 & 0.00000 \mathrm{E}+00 & 8.57488 \mathrm{E}-04 & 2.45929 \mathrm{E}-01 & 3.19836 \mathrm{E}-02 \\ 0.00000 \mathrm{E}+00 & 0.00000 \mathrm{E}+00 & 0.00000 \mathrm{E}+00 & 0.00000 \mathrm{E}+00 & 7.91005 \mathrm{E}-02 & 2.12477 \mathrm{E}-01 \\ \mathrm{P} 1 \mathrm{SCATTERING} & & & & & \\ 2.11391 \mathrm{E}-02 & -3.26180 \mathrm{E}-03 & 1.41419 \mathrm{E}-08 & 0.00000 \mathrm{E}+00 & 0.00000 \mathrm{E}+00 & 0.00000 \mathrm{E}+00 \\ 0.00000 \mathrm{E}+00 & 1.85920 \mathrm{E}-02 & -2.33974 \mathrm{E}-03 & 0.00000 \mathrm{E}+00 & 0.00000 \mathrm{E}+00 & 0.00000 \mathrm{E}+00 \\ 0.00000 \mathrm{E}+00 & 0.00000 \mathrm{E}+00 & 1.66799 \mathrm{E}-02 & -1.68066 \mathrm{E}-03 & 0.00000 \mathrm{E}+00 & 0.00000 \mathrm{E}+00 \\ 0.00000 \mathrm{E}+00 & 0.00000 \mathrm{E}+00 & 1.97098 \mathrm{E}-04 & 1.14028 \mathrm{E}-02 & -8.99168 \mathrm{E}-03 & 0.00000 \mathrm{E}+00 \\ 0.00000 \mathrm{E}+00 & 0.00000 \mathrm{E}+00 & 0.00000 \mathrm{E}+00 & -7.25913 \mathrm{E}-05 & -2.99652 \mathrm{E}-02 & -7.69676 \mathrm{E}-03 \\ 0.00000 \mathrm{E}+00 & 0.00000 \mathrm{E}+00 & 0.00000 \mathrm{E}+00 & 0.00000 \mathrm{E}+00 & -1.88585 \mathrm{E}-02 & -5.01497 \mathrm{E}-02\end{array}$

Graphite in fuel blocks adjacent to control rods (lavender blocks)

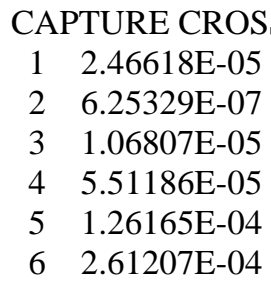

P0 SCATTERING

$\begin{array}{llccccc}2.29282 \mathrm{E}-01 & 1.67020 \mathrm{E}-02 & 1.72950 \mathrm{E}-08 & 0.00000 \mathrm{E}+00 & 0.00000 \mathrm{E}+00 & 0.00000 \mathrm{E}+00 \\ 0.00000 \mathrm{E}+00 & 3.90790 \mathrm{E}-01 & 1.17878 \mathrm{E}-02 & 0.00000 \mathrm{E}+00 & 0.00000 \mathrm{E}+00 & 0.00000 \mathrm{E}+00 \\ 0.00000 \mathrm{E}+00 & 0.00000 \mathrm{E}+00 & 4.04629 \mathrm{E}-01 & 8.72808 \mathrm{E}-03 & 0.00000 \mathrm{E}+00 & 0.00000 \mathrm{E}+00 \\ 0.00000 \mathrm{E}+00 & 0.00000 \mathrm{E}+00 & 8.97061 \mathrm{E}-04 & 3.58670 \mathrm{E}-01 & 5.43309 \mathrm{E}-02 & 0.00000 \mathrm{E}+00 \\ 0.00000 \mathrm{E}+00 & 0.00000 \mathrm{E}+00 & 0.00000 \mathrm{E}+00 & 1.27961 \mathrm{E}-03 & 3.67219 \mathrm{E}-01 & 4.78252 \mathrm{E}-02 \\ 0.00000 \mathrm{E}+00 & 0.00000 \mathrm{E}+00 & 0.00000 \mathrm{E}+00 & 0.00000 \mathrm{E}+00 & 1.17622 \mathrm{E}-01 & 3.15795 \mathrm{E}-01 \\ \text { P1 SCATTERING } & & & & & \\ \text { 3.00985E-02 } & -4.62757 \mathrm{E}-03 & 6.15086 \mathrm{E}-09 & 0.00000 \mathrm{E}+00 & 0.00000 \mathrm{E}+00 & 0.00000 \mathrm{E}+00\end{array}$




$\begin{array}{cccccc}0.00000 \mathrm{E}+00 & 2.76703 \mathrm{E}-02 & -3.47641 \mathrm{E}-03 & 0.00000 \mathrm{E}+00 & 0.00000 \mathrm{E}+00 & 0.00000 \mathrm{E}+00 \\ 0.00000 \mathrm{E}+00 & 0.00000 \mathrm{E}+00 & 2.43867 \mathrm{E}-02 & -2.46748 \mathrm{E}-03 & 0.00000 \mathrm{E}+00 & 0.00000 \mathrm{E}+00 \\ 0.00000 \mathrm{E}+00 & 0.00000 \mathrm{E}+00 & 2.97456 \mathrm{E}-04 & 1.71114 \mathrm{E}-02 & -1.34884 \mathrm{E}-02 & 0.00000 \mathrm{E}+00 \\ 0.00000 \mathrm{E}+00 & 0.00000 \mathrm{E}+00 & 0.00000 \mathrm{E}+00 & -1.10032 \mathrm{E}-04 & -4.46997 \mathrm{E}-02 & -1.15093 \mathrm{E}-02 \\ 0.00000 \mathrm{E}+00 & 0.00000 \mathrm{E}+00 & 0.00000 \mathrm{E}+00 & 0.00000 \mathrm{E}+00 & -2.80524 \mathrm{E}-02 & -7.45337 \mathrm{E}-02\end{array}$

Structural reflector block graphite (dark gray blocks)

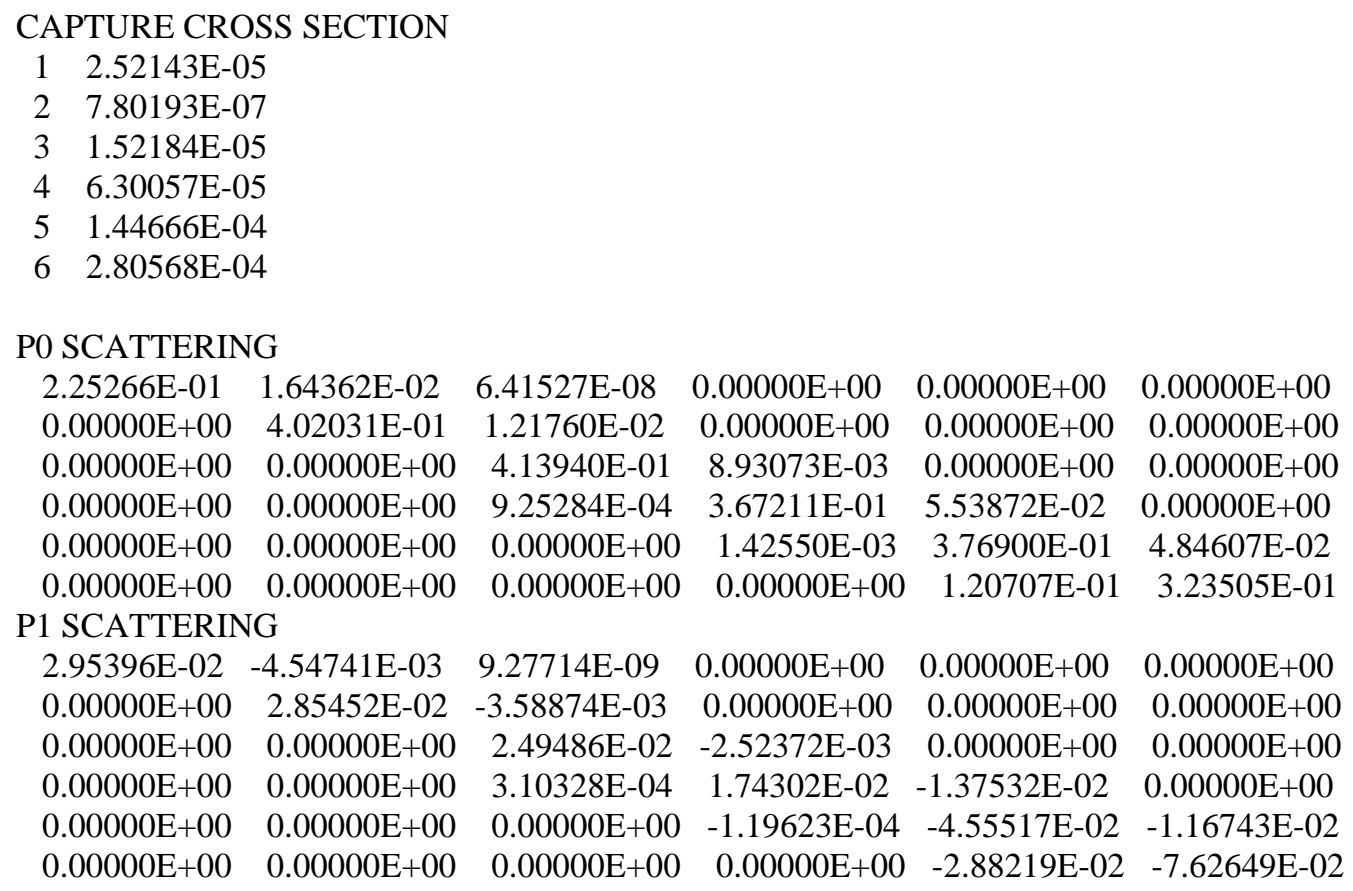

\section{APPENDIX B: PIN FISSION DENSITY FIGURES}

Fission density values at the pin level are presented in this appendix.

Table B1. Block-wide pin fission density values, 3-D stylized ARO core, axial layer 5 and 6

\begin{tabular}{|c|c|c|c|c|c|c|c|}
\hline$\#$ & 1 & 2 & 3 & 4 & 5 & 6 & 7 \\
\hline 1 & & 1.0347 & & 0.9082 & 0.9202 & 1.1145 & 0.7790 \\
\hline 2 & & 1.0073 & & 0.9951 & 1.0014 & 1.2840 & 0.8741 \\
\hline 3 & & 0.9695 & & 1.0073 & 1.0195 & 1.3369 & 0.9050 \\
\hline 4 & & 0.8939 & & 0.9976 & 1.0065 & 1.3995 & 0.9460 \\
\hline 5 & & 0.8466 & & 0.9734 & 0.9810 & 1.4156 & 0.9659 \\
\hline 6 & & 0.7265 & & 0.8783 & 0.8778 & 1.3772 & 0.9707 \\
\hline 7 & & 1.2543 & & 0.9182 & 0.9333 & 0.9471 & 0.7780 \\
\hline 8 & & 1.0839 & & 0.9588 & 0.9690 & 1.0972 & 0.8247 \\
\hline 9 & & 1.0427 & & 0.9843 & 0.9924 & 1.1636 & 0.8590 \\
\hline 10 & & 0.9610 & & 1.0030 & 1.0102 & 1.2479 & 0.9088 \\
\hline 11 & & 0.9177 & & 0.9967 & 1.0027 & 1.2763 & 0.9308 \\
\hline 12 & & 0.8291 & & 0.9576 & 0.9634 & 1.3131 & 0.9762 \\
\hline 13 & & 0.7796 & & 0.9261 & 0.9301 & 1.3173 & 1.0020 \\
\hline 14 & & 0.7029 & & 0.8683 & 0.8689 & 1.3751 & 1.1475 \\
\hline
\end{tabular}




\begin{tabular}{|c|c|c|c|c|c|}
\hline 15 & 1.2586 & 0.9673 & 0.9803 & 0.9744 & 0.8207 \\
\hline 16 & 1.1521 & 0.9623 & 0.9711 & 1.0056 & 0.8232 \\
\hline 17 & 1.0503 & 0.9940 & 1.0028 & 1.1064 & 0.8730 \\
\hline 18 & 1.0002 & 0.9981 & 1.0052 & 1.1419 & 0.8966 \\
\hline 19 & 0.9053 & 0.9868 & 0.9947 & 1.1954 & 0.9406 \\
\hline 20 & 0.8632 & 0.9723 & 0.9784 & 1.2202 & 0.9682 \\
\hline 21 & 0.7740 & 0.9224 & 0.9281 & 1.2473 & 1.0354 \\
\hline 22 & 0.7533 & 0.9155 & 0.9218 & 1.3180 & 1.1339 \\
\hline 23 & 1.4857 & 1.0220 & 1.0394 & 0.9411 & 0.8616 \\
\hline 24 & 1.3537 & 1.0015 & 1.0157 & 0.9471 & 0.8484 \\
\hline 25 & 1.1869 & 0.9941 & 1.0026 & 0.9992 & 0.8546 \\
\hline 26 & 1.1146 & 0.9976 & 1.0055 & 1.0286 & 0.8694 \\
\hline 27 & 0.9987 & 1.0020 & 1.0094 & 1.0922 & 0.9092 \\
\hline 28 & 0.9467 & 0.9954 & 1.0046 & 1.1196 & 0.9299 \\
\hline 29 & 0.8522 & 0.9695 & 0.9785 & 1.1625 & 0.9866 \\
\hline 30 & 0.8167 & 0.9558 & 0.9619 & 1.1962 & 1.0341 \\
\hline 31 & 0.7737 & 0.9415 & 0.9472 & 1.3203 & 1.2061 \\
\hline 32 & 0.7691 & 0.9511 & 0.9558 & 1.4242 & 1.3360 \\
\hline 33 & 1.5693 & 1.0508 & 1.0627 & 0.9232 & 0.8804 \\
\hline 34 & & 1.0136 & 1.0233 & 0.9368 & 0.8601 \\
\hline 35 & & 1.0078 & 1.0144 & 0.9532 & 0.8599 \\
\hline 36 & 1.1408 & 1.0090 & 1.0175 & 1.0057 & 0.8888 \\
\hline 37 & 1.0651 & 1.0096 & 1.0158 & 1.0302 & 0.9073 \\
\hline 38 & 0.9386 & 0.9973 & 1.0059 & 1.0793 & 0.9483 \\
\hline 39 & 0.8897 & 0.9891 & 0.9960 & 1.1048 & 0.9795 \\
\hline 40 & 0.8153 & 0.9635 & 0.9686 & 1.1704 & 1.0717 \\
\hline 41 & 0.7952 & 0.9573 & 0.9632 & 1.2316 & 1.1529 \\
\hline 42 & 0.7789 & 0.9692 & 0.9733 & 1.4226 & 1.4000 \\
\hline 43 & & 1.0415 & 1.0528 & 0.9004 & 0.8758 \\
\hline 44 & & 1.0282 & 1.0374 & 0.9076 & 0.8674 \\
\hline 45 & & 1.0205 & 1.0271 & 0.9403 & 0.8773 \\
\hline 46 & & 1.0166 & 1.0240 & 0.9615 & 0.8881 \\
\hline 47 & 1.0659 & 1.0166 & 1.0229 & 1.0069 & 0.9240 \\
\hline 48 & 0.9894 & 1.0122 & 1.0198 & 1.0311 & 0.9463 \\
\hline 49 & 0.8814 & 0.9899 & 0.9965 & 1.0779 & 1.0043 \\
\hline 50 & 0.8436 & 0.9775 & 0.9869 & 1.1142 & 1.0502 \\
\hline 51 & 0.7958 & 0.9651 & 0.9692 & 1.2237 & 1.1937 \\
\hline 52 & 0.7806 & 0.9640 & 0.9689 & 1.3143 & 1.3052 \\
\hline 53 & 1.6590 & 1.0606 & 1.0718 & 0.8766 & 0.8860 \\
\hline 54 & & 1.0459 & 1.0533 & 0.8789 & 0.8770 \\
\hline 55 & & 1.0330 & 1.0367 & 0.8954 & 0.8738 \\
\hline 56 & & 1.0313 & 1.0330 & 0.9098 & 0.8793 \\
\hline 57 & & 1.0313 & 1.0369 & 0.9516 & 0.9072 \\
\hline 58 & 1.1440 & 1.0318 & 1.0350 & 0.9707 & 0.9243 \\
\hline 59 & 0.9771 & 1.0186 & 1.0250 & 1.0144 & 0.9666 \\
\hline
\end{tabular}




\begin{tabular}{|c|c|c|c|c|c|c|c|}
\hline 60 & & 0.9180 & & 1.0093 & 1.0153 & 1.0389 & 0.9956 \\
\hline 61 & & 0.8366 & & 0.9814 & 0.9878 & 1.0989 & 1.0768 \\
\hline 62 & & 0.8095 & & 0.9706 & 0.9787 & 1.1533 & 1.1416 \\
\hline 63 & 0.8532 & 0.7779 & 1.3103 & 0.9628 & 0.9678 & 1.3085 & 1.3357 \\
\hline 64 & 0.8568 & 0.7698 & 1.4174 & 0.9641 & 0.9685 & 1.4152 & 1.4623 \\
\hline 65 & & 1.6906 & & 1.0466 & 1.0547 & 0.8450 & 0.8725 \\
\hline 66 & & & & 1.0389 & 1.0456 & 0.8611 & 0.8723 \\
\hline 67 & & & & 1.0374 & 1.0423 & 0.8720 & 0.8741 \\
\hline 68 & & & & 1.0394 & 1.0424 & 0.9014 & 0.8930 \\
\hline 69 & & & & 1.0411 & 1.0447 & 0.9212 & 0.9057 \\
\hline 70 & & 1.1128 & & 1.0478 & 1.0543 & 0.9711 & 0.9504 \\
\hline 71 & & 1.0266 & & 1.0424 & 1.0485 & 0.9911 & 0.9709 \\
\hline 72 & & 0.9039 & & 1.0100 & 1.0168 & 1.0267 & 1.0171 \\
\hline 73 & & 0.8601 & & 0.9968 & 1.0022 & 1.0568 & 1.0516 \\
\hline 74 & 0.8637 & 0.8004 & 1.1585 & 0.9706 & 0.9740 & 1.1481 & 1.1705 \\
\hline 75 & 0.8549 & 0.7799 & 1.2169 & 0.9601 & 0.9666 & 1.2130 & 1.2525 \\
\hline 76 & 0.8413 & 0.7504 & 1.3806 & 0.9432 & 0.9470 & 1.4004 & 1.4777 \\
\hline 77 & & 1.5651 & & 1.0309 & 1.0340 & 0.8252 & 0.8577 \\
\hline 78 & & & & 1.0361 & 1.0394 & 0.8383 & 0.8646 \\
\hline 79 & & & & 1.0409 & 1.0422 & 0.8644 & 0.8774 \\
\hline 80 & & & & 1.0430 & 1.0474 & 0.8794 & 0.8885 \\
\hline 81 & & & & 1.0575 & 1.0619 & 0.9244 & 0.9270 \\
\hline 82 & & 1.1899 & & 1.0722 & 1.0759 & 0.9545 & 0.9567 \\
\hline 83 & & 1.0099 & & 1.0565 & 1.0631 & 0.9937 & 1.0013 \\
\hline 84 & & 0.9395 & & 1.0331 & 1.0400 & 1.0061 & 1.0146 \\
\hline 85 & 0.8913 & 0.8463 & 1.0807 & 0.9915 & 0.9987 & 1.0513 & 1.0783 \\
\hline 86 & 0.8736 & 0.8121 & 1.1077 & 0.9767 & 0.9812 & 1.0894 & 1.1247 \\
\hline 87 & 0.8474 & 0.7677 & 1.1942 & 0.9502 & 0.9537 & 1.2127 & 1.2782 \\
\hline 88 & 0.8324 & 0.7454 & 1.2584 & 0.9324 & 0.9383 & 1.2920 & 1.3724 \\
\hline 89 & & 1.6645 & & 0.9609 & 0.9624 & 0.7500 & 0.7944 \\
\hline 90 & & 1.5766 & & 1.0046 & 1.0045 & 0.7915 & 0.8328 \\
\hline 91 & & & & 1.0311 & 1.0312 & 0.8286 & 0.8621 \\
\hline 92 & & & & 1.0428 & 1.0432 & 0.8450 & 0.8744 \\
\hline 93 & & & & 1.0510 & 1.0537 & 0.8773 & 0.9002 \\
\hline 94 & & & & 1.0643 & 1.0656 & 0.9013 & 0.9236 \\
\hline 95 & 0.9359 & 0.9131 & 1.0543 & 1.0314 & 1.0359 & 1.0014 & 1.0337 \\
\hline 96 & 0.9069 & 0.8638 & 1.0561 & 1.0055 & 1.0099 & 1.0198 & 1.0569 \\
\hline 97 & 0.8693 & 0.7994 & 1.0985 & 0.9714 & 0.9741 & 1.0960 & 1.1521 \\
\hline 98 & 0.8510 & 0.7724 & 1.1265 & 0.9510 & 0.9548 & 1.1463 & 1.2142 \\
\hline 99 & 0.8065 & 0.7191 & 1.1962 & 0.9037 & 0.9080 & 1.2803 & 1.3804 \\
\hline 100 & 0.7614 & 0.6732 & 1.2175 & 0.8533 & 0.8579 & 1.3335 & 1.4434 \\
\hline 101 & & 1.4884 & & 1.0034 & 0.9999 & 0.7855 & 0.8318 \\
\hline 102 & & & & 1.0282 & 1.0282 & 0.8133 & 0.8589 \\
\hline 103 & & & & 1.0444 & 1.0395 & 0.8407 & 0.8809 \\
\hline 104 & & 1.4308 & & 1.0524 & 1.0499 & 0.8562 & 0.8952 \\
\hline
\end{tabular}




\begin{tabular}{|c|c|c|c|c|c|c|c|}
\hline 105 & & 1.2157 & & 1.0822 & 1.0807 & 0.9078 & 0.9451 \\
\hline 106 & 0.9660 & 0.9411 & 1.0514 & 1.0554 & 1.0565 & 0.9859 & 1.0337 \\
\hline 107 & 0.9048 & 0.8448 & 1.0503 & 1.0005 & 1.0036 & 1.0212 & 1.0801 \\
\hline 108 & 0.8821 & 0.8113 & 1.0595 & 0.9798 & 0.9841 & 1.0563 & 1.1213 \\
\hline 109 & 0.8430 & 0.7590 & 1.0962 & 0.9430 & 0.9458 & 1.1631 & 1.2494 \\
\hline 110 & 0.8096 & 0.7232 & 1.0984 & 0.9057 & 0.9086 & 1.2059 & 1.3079 \\
\hline 111 & & 1.6689 & & 0.9676 & & 0.7407 & 0.7967 \\
\hline 112 & & 1.5789 & & 1.0133 & & 0.7797 & 0.8359 \\
\hline 113 & & 1.3854 & & 1.0353 & & 0.8144 & 0.8650 \\
\hline 114 & & 1.3210 & & 1.0447 & & 0.8287 & 0.8763 \\
\hline 115 & & 1.2329 & & 1.0558 & & 0.8537 & 0.9017 \\
\hline 116 & & 1.1830 & & 1.0687 & & 0.8787 & 0.9243 \\
\hline 117 & 0.9487 & 0.9010 & 1.0312 & 1.0347 & & 0.9760 & 1.0367 \\
\hline 118 & 0.9191 & 0.8591 & 1.0275 & 1.0088 & & 0.9954 & 1.0600 \\
\hline 119 & 0.8780 & 0.7980 & 1.0457 & 0.9750 & & 1.0722 & 1.1555 \\
\hline 120 & 0.8581 & 0.7686 & 1.0530 & 0.9514 & & 1.1246 & 1.2176 \\
\hline 121 & 0.8114 & 0.7186 & 1.0722 & 0.9048 & & 1.2606 & 1.3834 \\
\hline 122 & 0.7625 & 0.6740 & 1.0402 & 0.8539 & & 1.3138 & 1.4519 \\
\hline 123 & & 1.5690 & & 1.0448 & & 0.8021 & 0.8598 \\
\hline 124 & & 1.4456 & & 1.0450 & & 0.8111 & 0.8699 \\
\hline 125 & & 1.2736 & & 1.0460 & & 0.8268 & 0.8806 \\
\hline 126 & & 1.2180 & & 1.0503 & & 0.8386 & 0.8935 \\
\hline 127 & 1.0782 & 1.1162 & 0.9940 & 1.0663 & & 0.8753 & 0.9324 \\
\hline 128 & 1.0592 & 1.0739 & 1.0117 & 1.0775 & & 0.9029 & 0.9614 \\
\hline 129 & 1.0043 & 0.9688 & 1.0250 & 1.0655 & & 0.9422 & 1.0050 \\
\hline 130 & 0.9653 & 0.9131 & 1.0180 & 1.0396 & & 0.9514 & 1.0188 \\
\hline 131 & 0.9089 & 0.8354 & 1.0120 & 0.9987 & & 1.0029 & 1.0803 \\
\hline 132 & 0.8891 & 0.8061 & 1.0177 & 0.9810 & & 1.0444 & 1.1295 \\
\hline 133 & 0.8579 & 0.7640 & 1.0398 & 0.9531 & & 1.1705 & 1.2805 \\
\hline 134 & 0.8407 & 0.7453 & 1.0468 & 0.9358 & & 1.2563 & 1.3804 \\
\hline 135 & & 1.6896 & & 1.0648 & & 0.8119 & 0.8768 \\
\hline 136 & & 1.4072 & & 1.0533 & & 0.8164 & 0.8772 \\
\hline 137 & & 1.3080 & & 1.0489 & & 0.8185 & 0.8816 \\
\hline 138 & 1.1430 & 1.1622 & 0.9677 & 1.0482 & & 0.8370 & 0.8965 \\
\hline 139 & 1.0987 & 1.1076 & 0.9764 & 1.0540 & & 0.8494 & 0.9118 \\
\hline 140 & 1.0381 & 1.0130 & 0.9942 & 1.0575 & & 0.8902 & 0.9556 \\
\hline 141 & 1.0089 & 0.9645 & 0.9990 & 1.0537 & & 0.9098 & 0.9775 \\
\hline 142 & 0.9475 & 0.8782 & 0.9945 & 1.0190 & & 0.9479 & 1.0225 \\
\hline 143 & 0.9223 & 0.8424 & 0.9926 & 1.0037 & & 0.9794 & 1.0595 \\
\hline 144 & 0.8850 & 0.7943 & 1.0034 & 0.9767 & & 1.0788 & 1.1753 \\
\hline 145 & 0.8728 & 0.7766 & 1.0115 & 0.9646 & & 1.1472 & 1.2588 \\
\hline 146 & 0.8529 & 0.7500 & 1.0393 & 0.9473 & & 1.3417 & 1.4871 \\
\hline 147 & & 1.6509 & & 1.0840 & & 0.8268 & 0.8933 \\
\hline 148 & & 1.4952 & & 1.0645 & & 0.8175 & 0.8854 \\
\hline 149 & 1.2731 & 1.2577 & 0.9572 & 1.0463 & & 0.8163 & 0.8823 \\
\hline
\end{tabular}




\begin{tabular}{|c|c|c|c|c|c|c|}
\hline 150 & 1.1930 & 1.1782 & 0.9571 & 1.0418 & 0.8226 & 0.8873 \\
\hline 151 & 1.0895 & 1.0559 & 0.9711 & 1.0453 & 0.8466 & 0.9150 \\
\hline 152 & 1.0510 & 1.0067 & 0.9740 & 1.0437 & 0.8635 & 0.9326 \\
\hline 153 & 0.9868 & 0.9206 & 0.9789 & 1.0295 & 0.9014 & 0.9754 \\
\hline 154 & 0.9589 & 0.8840 & 0.9781 & 1.0186 & 0.9269 & 1.0024 \\
\hline 155 & 0.9109 & 0.8226 & 0.9761 & 0.9905 & 0.9941 & 1.0870 \\
\hline 156 & 0.8965 & 0.8018 & 0.9828 & 0.9791 & 1.0518 & 1.1500 \\
\hline 157 & 0.8768 & 0.7745 & 1.0013 & 0.9679 & 1.2158 & 1.3412 \\
\hline 158 & 0.8749 & 0.7698 & 1.0204 & 0.9686 & 1.3219 & 1.4682 \\
\hline 159 & 1.5007 & 1.4473 & 0.9637 & 1.0610 & 0.8158 & 0.8837 \\
\hline 160 & 1.3630 & 1.3113 & 0.9562 & 1.0450 & 0.8127 & 0.8780 \\
\hline 161 & 1.1807 & 1.1313 & 0.9533 & 1.0345 & 0.8192 & 0.8869 \\
\hline 162 & 1.1176 & 1.0659 & 0.9571 & 1.0347 & 0.8299 & 0.8982 \\
\hline 163 & 1.0363 & 0.9653 & 0.9645 & 1.0314 & 0.8621 & 0.9348 \\
\hline 164 & 0.9998 & 0.9262 & 0.9645 & 1.0233 & 0.8801 & 0.9567 \\
\hline 165 & 0.9439 & 0.8538 & 0.9620 & 1.0033 & 0.9308 & 1.0154 \\
\hline 166 & 0.9204 & 0.8270 & 0.9621 & 0.9907 & 0.9715 & 1.0593 \\
\hline 167 & 0.8861 & 0.7873 & 0.9681 & 0.9722 & 1.0945 & 1.2016 \\
\hline 168 & 0.8825 & 0.7782 & 0.9801 & 0.9712 & 1.1855 & 1.3106 \\
\hline 169 & 1.6532 & 1.5530 & 0.9716 & 1.0749 & 0.8214 & 0.8907 \\
\hline 170 & 1.3348 & 1.2539 & 0.9457 & 1.0337 & 0.8038 & 0.8698 \\
\hline 171 & 1.2312 & 1.1547 & 0.9405 & 1.0234 & 0.8039 & 0.8720 \\
\hline 172 & 1.1043 & 1.0216 & 0.9484 & 1.0256 & 0.8301 & 0.8994 \\
\hline 173 & 1.0585 & 0.9728 & 0.9539 & 1.0240 & 0.8443 & 0.9169 \\
\hline 174 & 0.9873 & 0.8934 & 0.9520 & 1.0121 & 0.8823 & 0.9621 \\
\hline 175 & 0.9565 & 0.8598 & 0.9520 & 1.0034 & 0.9089 & 0.9929 \\
\hline 176 & 0.9040 & 0.8029 & 0.9409 & 0.9720 & 0.9903 & 1.0859 \\
\hline 177 & 0.8905 & 0.7857 & 0.9451 & 0.9685 & 1.0569 & 1.1643 \\
\hline 178 & 0.8867 & 0.7777 & 0.9736 & 0.9751 & 1.2674 & 1.4044 \\
\hline 179 & 1.6031 & 1.4741 & 0.9522 & 1.0499 & 0.8046 & 0.8730 \\
\hline 180 & 1.4274 & 1.3170 & 0.9354 & 1.0268 & 0.7937 & 0.8596 \\
\hline 181 & 1.2002 & 1.1003 & 0.9320 & 1.0129 & 0.8003 & 0.8674 \\
\hline 182 & 1.1333 & 1.0330 & 0.9369 & 1.0139 & 0.8113 & 0.8811 \\
\hline 183 & 1.0439 & 0.9413 & 0.9488 & 1.0174 & 0.8476 & 0.9237 \\
\hline 184 & 1.0070 & 0.9033 & 0.9477 & 1.0118 & 0.8677 & 0.9455 \\
\hline 185 & 0.9392 & 0.8321 & 0.9332 & 0.9829 & 0.9154 & 1.0028 \\
\hline 186 & 0.9113 & 0.8034 & 0.9251 & 0.9705 & 0.9558 & 1.0518 \\
\hline 187 & 0.8765 & 0.7693 & 0.9258 & 0.9541 & 1.1031 & 1.2183 \\
\hline 188 & 0.8786 & 0.7659 & 0.9395 & 0.9621 & 1.2161 & 1.3452 \\
\hline 189 & 1.3567 & 1.2268 & 0.9068 & 0.9900 & 0.7700 & 0.8362 \\
\hline 190 & 1.2242 & 1.1054 & 0.9039 & 0.9832 & 0.7711 & 0.8362 \\
\hline 191 & 1.1185 & 1.0004 & 0.9369 & 1.0131 & 0.8150 & 0.8888 \\
\hline 192 & 1.0721 & 0.9555 & 0.9445 & 1.0155 & 0.8349 & 0.9109 \\
\hline 193 & 0.9972 & 0.8808 & 0.9398 & 1.0044 & 0.8753 & 0.9577 \\
\hline 194 & 0.9600 & 0.8445 & 0.9305 & 0.9897 & 0.8993 & 0.9851 \\
\hline
\end{tabular}




\begin{tabular}{|l|l|l|l|l|l|l|l|}
\hline 195 & 0.8767 & 0.7661 & 0.8883 & 0.9358 & & 0.9592 & 1.0514 \\
\hline 196 & 0.8614 & 0.7516 & 0.8906 & 0.9295 & & 1.0436 & 1.1493 \\
\hline 197 & 1.3993 & 1.2525 & 0.8641 & 0.9438 & & 0.7287 & 0.7903 \\
\hline 198 & 1.1933 & 1.0574 & 0.9032 & 0.9817 & & 0.7733 & 0.8392 \\
\hline 199 & 1.1504 & 1.0134 & 0.9280 & 1.0057 & & 0.8027 & 0.8741 \\
\hline 200 & 1.0671 & 0.9370 & 0.9459 & 1.0209 & & 0.8477 & 0.9255 \\
\hline 201 & 1.0270 & 0.8985 & 0.9460 & 1.0139 & & 0.8692 & 0.9484 \\
\hline 202 & 0.9423 & 0.8190 & 0.9147 & 0.9751 & & 0.9082 & 0.9985 \\
\hline 203 & 0.8925 & 0.7742 & 0.8857 & 0.9416 & & 0.9331 & 1.0201 \\
\hline 204 & 0.8121 & 0.7025 & 0.8380 & 0.8799 & & 1.0615 & 1.1686 \\
\hline 205 & 1.1746 & 1.0286 & 0.8580 & 0.9315 & & 0.7304 & 0.7927 \\
\hline 206 & 1.1482 & 0.9919 & 0.9397 & 1.0160 & & 0.8169 & 0.8886 \\
\hline 207 & 1.1075 & 0.9577 & 0.9525 & 1.0293 & & 0.8451 & 0.9206 \\
\hline 208 & 1.0283 & 0.8864 & 0.9481 & 1.0191 & & 0.8861 & 0.9660 \\
\hline 209 & 0.9762 & 0.8410 & 0.9278 & 0.9934 & & 0.9014 & 0.9882 \\
\hline 210 & 0.8425 & 0.7263 & 0.8348 & 0.8913 & & 0.9040 & 0.9896 \\
\hline
\end{tabular}

Table B2. Block-wide pin fission density values, 3-D stylized SRI core, axial layer 1 and 10

\begin{tabular}{|c|c|c|c|c|c|c|c|}
\hline$\#$ & 1 & 2 & 3 & 4 & 5 & 6 & 7 \\
\hline 1 & & 1.0234 & & 0.9122 & 0.9260 & 1.1244 & 0.8210 \\
\hline 2 & & 1.0086 & & 0.9922 & 1.0090 & 1.2662 & 0.9011 \\
\hline 3 & & 0.9781 & & 1.0024 & 1.0209 & 1.3019 & 0.9209 \\
\hline 4 & & 0.9082 & & 0.9876 & 1.0044 & 1.3266 & 0.9369 \\
\hline 5 & & 0.8626 & & 0.9614 & 0.9765 & 1.3217 & 0.9378 \\
\hline 6 & & 0.7433 & & 0.8543 & 0.8687 & 1.2560 & 0.9081 \\
\hline 7 & & 1.2208 & & 0.9284 & 0.9434 & 0.9882 & 0.8348 \\
\hline 8 & & 1.0739 & & 0.9635 & 0.9776 & 1.1094 & 0.8687 \\
\hline 9 & & 1.0403 & & 0.9856 & 1.0001 & 1.1608 & 0.8922 \\
\hline 10 & & 0.9674 & & 0.9967 & 1.0114 & 1.2138 & 0.9200 \\
\hline 11 & & 0.9297 & & 0.9884 & 1.0036 & 1.2255 & 0.9297 \\
\hline 12 & & 0.8469 & & 0.9459 & 0.9592 & 1.2261 & 0.9410 \\
\hline 13 & & 0.7991 & & 0.9075 & 0.9214 & 1.2116 & 0.9467 \\
\hline 14 & & 0.7224 & & 0.8387 & 0.8542 & 1.2186 & 1.0315 \\
\hline 15 & & 1.2306 & & 0.9792 & 0.9922 & 1.0156 & 0.8814 \\
\hline 16 & & 1.1374 & & 0.9702 & 0.9832 & 1.0326 & 0.8730 \\
\hline 17 & & 1.0473 & & 0.9960 & 1.0100 & 1.1085 & 0.9043 \\
\hline 18 & & 1.0042 & & 0.9977 & 1.0128 & 1.1300 & 0.9155 \\
\hline 19 & & 0.9186 & & 0.9802 & 0.9954 & 1.1517 & 0.9328 \\
\hline 20 & & 0.8780 & & 0.9626 & 0.9773 & 1.1555 & 0.9441 \\
\hline 21 & & 0.7931 & & 0.9037 & 0.9173 & 1.1437 & 0.9710 \\
\hline 22 & & 0.7755 & & 0.8939 & 0.9068 & 1.1844 & 1.0332 \\
\hline 23 & & 1.4405 & & 1.0375 & 1.0509 & 1.0065 & 0.9335 \\
\hline 24 & & 1.3230 & & 1.0162 & 1.0299 & 1.0029 & 0.9135 \\
\hline
\end{tabular}




\begin{tabular}{|c|c|c|c|c|c|c|c|}
\hline 25 & & 1.1763 & & 1.0020 & 1.0136 & 1.0288 & 0.9029 \\
\hline 26 & & 1.1074 & & 1.0014 & 1.0142 & 1.0483 & 0.9074 \\
\hline 27 & & 1.0038 & & 1.0006 & 1.0139 & 1.0817 & 0.9252 \\
\hline 28 & & 0.9561 & & 0.9915 & 1.0050 & 1.0904 & 0.9339 \\
\hline 29 & & 0.8705 & & 0.9593 & 0.9736 & 1.1002 & 0.9553 \\
\hline 30 & & 0.8360 & & 0.9427 & 0.9549 & 1.1118 & 0.9801 \\
\hline 31 & & 0.7938 & & 0.9186 & 0.9319 & 1.1802 & 1.0876 \\
\hline 32 & & 0.7910 & & 0.9203 & 0.9330 & 1.2461 & 1.1766 \\
\hline 33 & & 1.5181 & & 1.0647 & 1.0774 & 0.9979 & 0.9602 \\
\hline 34 & & & & 1.0270 & 1.0377 & 0.9913 & 0.9237 \\
\hline 35 & & & & 1.0172 & 1.0276 & 0.9944 & 0.9150 \\
\hline 36 & & 1.1338 & & 1.0154 & 1.0264 & 1.0248 & 0.9226 \\
\hline 37 & & 1.0658 & & 1.0109 & 1.0228 & 1.0339 & 0.9268 \\
\hline 38 & & 0.9499 & & 0.9930 & 1.0054 & 1.0507 & 0.9438 \\
\hline 39 & & 0.9056 & & 0.9807 & 0.9925 & 1.0608 & 0.9580 \\
\hline 40 & & 0.8358 & & 0.9453 & 0.9586 & 1.0835 & 1.0053 \\
\hline 41 & & 0.8162 & & 0.9366 & 0.9502 & 1.1194 & 1.0559 \\
\hline 42 & & 0.8026 & & 0.9324 & 0.9482 & 1.2403 & 1.2208 \\
\hline 43 & & & & 1.0568 & 1.0669 & 0.9765 & 0.9510 \\
\hline 44 & & & & 1.0440 & 1.0515 & 0.9724 & 0.9366 \\
\hline 45 & & & & 1.0317 & 1.0391 & 0.9836 & 0.9278 \\
\hline 46 & & & & 1.0269 & 1.0350 & 0.9922 & 0.9292 \\
\hline 47 & & 1.0669 & & 1.0183 & 1.0288 & 1.0115 & 0.9410 \\
\hline 48 & & 0.9957 & & 1.0089 & 1.0203 & 1.0203 & 0.9500 \\
\hline 49 & & 0.8980 & & 0.9823 & 0.9930 & 1.0322 & 0.9733 \\
\hline 50 & & 0.8635 & & 0.9663 & 0.9797 & 1.0479 & 0.9979 \\
\hline 51 & & 0.8177 & & 0.9416 & 0.9547 & 1.1076 & 1.0854 \\
\hline 52 & & 0.8050 & & 0.9351 & 0.9493 & 1.1621 & 1.1569 \\
\hline 53 & & 1.5917 & & 1.0773 & 1.0853 & 0.9689 & 0.9720 \\
\hline 54 & & & & 1.0638 & 1.0712 & 0.9624 & 0.9576 \\
\hline 55 & & & & 1.0483 & 1.0532 & 0.9604 & 0.9393 \\
\hline 56 & & & & 1.0425 & 1.0472 & 0.9641 & 0.9366 \\
\hline 57 & & & & 1.0402 & 1.0471 & 0.9814 & 0.9438 \\
\hline 58 & & 1.1385 & & 1.0337 & 1.0424 & 0.9896 & 0.9488 \\
\hline 59 & & 0.9859 & & 1.0157 & 1.0263 & 1.0024 & 0.9639 \\
\hline 60 & & 0.9335 & & 1.0023 & 1.0113 & 1.0074 & 0.9757 \\
\hline 61 & & 0.8564 & & 0.9678 & 0.9777 & 1.0320 & 1.0181 \\
\hline 62 & & 0.8326 & & 0.9536 & 0.9635 & 1.0607 & 1.0564 \\
\hline 63 & 0.8626 & 0.8009 & 1.2068 & 0.9316 & 0.9455 & 1.1544 & 1.1807 \\
\hline 64 & 0.8622 & 0.7935 & 1.2859 & 0.9270 & 0.9404 & 1.2241 & 1.2667 \\
\hline 65 & & 1.6126 & & 1.0648 & 1.0701 & 0.9413 & 0.9589 \\
\hline 66 & & & & 1.0565 & 1.0607 & 0.9428 & 0.9486 \\
\hline 67 & & & & 1.0533 & 1.0567 & 0.9448 & 0.9452 \\
\hline 68 & & & & 1.0512 & 1.0550 & 0.9561 & 0.9456 \\
\hline 69 & & & & 1.0502 & 1.0563 & 0.9633 & 0.9497 \\
\hline
\end{tabular}




\begin{tabular}{|c|c|c|c|c|c|c|c|}
\hline 70 & & 1.1114 & & 1.0497 & 1.0567 & 0.9850 & 0.9685 \\
\hline 71 & & 1.0328 & & 1.0398 & 1.0470 & 0.9919 & 0.9780 \\
\hline 72 & & 0.9190 & & 1.0026 & 1.0111 & 0.9964 & 0.9912 \\
\hline 73 & & 0.8786 & & 0.9852 & 0.9934 & 1.0057 & 1.0089 \\
\hline 74 & 0.8769 & 0.8242 & 1.0939 & 0.9502 & 0.9602 & 1.0523 & 1.0760 \\
\hline 75 & 0.8645 & 0.8036 & 1.1358 & 0.9342 & 0.9449 & 1.0931 & 1.1294 \\
\hline 76 & 0.8446 & 0.7736 & 1.2546 & 0.9062 & 0.9186 & 1.2126 & 1.2794 \\
\hline 77 & & 1.5051 & & 1.0497 & 1.0514 & 0.9201 & 0.9432 \\
\hline 78 & & & & 1.0545 & 1.0555 & 0.9266 & 0.9461 \\
\hline 79 & & & & 1.0548 & 1.0565 & 0.9354 & 0.9468 \\
\hline 80 & & & & 1.0574 & 1.0605 & 0.9422 & 0.9489 \\
\hline 81 & & & & 1.0667 & 1.0713 & 0.9656 & 0.9671 \\
\hline 82 & & 1.1838 & & 1.0744 & 1.0797 & 0.9820 & 0.9850 \\
\hline 83 & & 1.0174 & & 1.0537 & 1.0607 & 0.9925 & 1.0010 \\
\hline 84 & & 0.9509 & & 1.0277 & 1.0341 & 0.9883 & 1.0003 \\
\hline 85 & 0.9047 & 0.8652 & 1.0448 & 0.9813 & 0.9886 & 0.9992 & 1.0239 \\
\hline 86 & 0.8875 & 0.8352 & 1.0606 & 0.9602 & 0.9696 & 1.0183 & 1.0540 \\
\hline 87 & 0.8564 & 0.7905 & 1.1184 & 0.9231 & 0.9329 & 1.0888 & 1.1470 \\
\hline 88 & 0.8402 & 0.7696 & 1.1607 & 0.9016 & 0.9118 & 1.1397 & 1.2126 \\
\hline 89 & & 1.5745 & & 0.9781 & 0.9761 & 0.8474 & 0.8801 \\
\hline 90 & & 1.5044 & & 1.0244 & 1.0230 & 0.8882 & 0.9211 \\
\hline 91 & & & & 1.0510 & 1.0488 & 0.9154 & 0.9412 \\
\hline 92 & & & & 1.0580 & 1.0560 & 0.9244 & 0.9481 \\
\hline 93 & & & & 1.0639 & 1.0644 & 0.9378 & 0.9572 \\
\hline 94 & & & & 1.0738 & 1.0759 & 0.9532 & 0.9706 \\
\hline 95 & 0.9480 & 0.9278 & 1.0381 & 1.0251 & 1.0288 & 0.9799 & 1.0143 \\
\hline 96 & 0.9218 & 0.8835 & 1.0322 & 0.9963 & 1.0029 & 0.9824 & 1.0202 \\
\hline 97 & 0.8832 & 0.8235 & 1.0514 & 0.9536 & 0.9609 & 1.0196 & 1.0738 \\
\hline 98 & 0.8628 & 0.7960 & 1.0647 & 0.9296 & 0.9376 & 1.0467 & 1.1116 \\
\hline 99 & 0.8151 & 0.7422 & 1.1066 & 0.8727 & 0.8802 & 1.1275 & 1.2135 \\
\hline 100 & 0.7653 & 0.6942 & 1.1120 & 0.8168 & 0.8250 & 1.1554 & 1.2512 \\
\hline 101 & & 1.4281 & & 1.0233 & 1.0175 & 0.8801 & 0.9163 \\
\hline 102 & & 1.3849 & & 1.0490 & 1.0446 & 0.9049 & 0.9391 \\
\hline 103 & & & & 1.0607 & 1.0569 & 0.9200 & 0.9491 \\
\hline 104 & & & & 1.0662 & 1.0643 & 0.9275 & 0.9579 \\
\hline 105 & & 1.2063 & & 1.0879 & 1.0895 & 0.9568 & 0.9877 \\
\hline 106 & 0.9765 & 0.9551 & 1.0443 & 1.0469 & 1.0490 & 0.9796 & 1.0215 \\
\hline 107 & 0.9178 & 0.8658 & 1.0246 & 0.9878 & 0.9923 & 0.9806 & 1.0353 \\
\hline 108 & 0.8959 & 0.8348 & 1.0265 & 0.9658 & 0.9713 & 0.9974 & 1.0578 \\
\hline 109 & 0.8563 & 0.7823 & 1.0421 & 0.9194 & 0.9260 & 1.0559 & 1.1387 \\
\hline 110 & 0.8218 & 0.7468 & 1.0360 & 0.8793 & 0.8859 & 1.0793 & 1.1699 \\
\hline 111 & & 1.5748 & & 0.9872 & & 0.8428 & 0.8833 \\
\hline 112 & & 1.5020 & & 1.0322 & & 0.8831 & 0.9238 \\
\hline 113 & & 1.3432 & & 1.0561 & & 0.9057 & 0.9448 \\
\hline 114 & & 1.2904 & & 1.0625 & & 0.9133 & 0.9505 \\
\hline
\end{tabular}




\begin{tabular}{|c|c|c|c|c|c|c|}
\hline 115 & & 1.2159 & & 1.0677 & 0.9236 & 0.9605 \\
\hline 116 & & 1.1724 & & 1.0779 & 0.9360 & 0.9750 \\
\hline 117 & 0.9607 & 0.9197 & 1.0250 & 1.0277 & 0.9652 & 1.0195 \\
\hline 118 & 0.9326 & 0.8785 & 1.0142 & 1.0003 & 0.9683 & 1.0271 \\
\hline 119 & 0.8919 & 0.8213 & 1.0139 & 0.9577 & 1.0094 & 1.0840 \\
\hline 120 & 0.8707 & 0.7950 & 1.0138 & 0.9337 & 1.0379 & 1.1230 \\
\hline 121 & 0.8205 & 0.7421 & 1.0106 & 0.8753 & 1.1234 & 1.2321 \\
\hline 122 & 0.7711 & 0.6946 & 0.9716 & 0.8195 & 1.1552 & 1.2748 \\
\hline 123 & & 1.4948 & & 1.0626 & 0.9057 & 0.9514 \\
\hline 124 & & 1.3898 & & 1.0657 & 0.9091 & 0.9517 \\
\hline 125 & & 1.2473 & & 1.0645 & 0.9095 & 0.9524 \\
\hline 126 & & 1.1992 & & 1.0661 & 0.9149 & 0.9577 \\
\hline 127 & 1.0767 & 1.1100 & 1.0226 & 1.0750 & 0.9305 & 0.9772 \\
\hline 128 & 1.0621 & 1.0738 & 1.0370 & 1.0832 & 0.9463 & 0.9958 \\
\hline 129 & 1.0120 & 0.9797 & 1.0338 & 1.0648 & 0.9560 & 1.0135 \\
\hline 130 & 0.9764 & 0.9292 & 1.0191 & 1.0363 & 0.9536 & 1.0147 \\
\hline 131 & 0.9246 & 0.8577 & 1.0009 & 0.9896 & 0.9705 & 1.0416 \\
\hline 132 & 0.9042 & 0.8308 & 0.9967 & 0.9693 & 0.9937 & 1.0722 \\
\hline 133 & 0.8712 & 0.7900 & 1.0007 & 0.9322 & 1.0734 & 1.1739 \\
\hline 134 & 0.8527 & 0.7692 & 0.9989 & 0.9097 & 1.1308 & 1.2462 \\
\hline 135 & & 1.5995 & & 1.0856 & 0.9225 & 0.9695 \\
\hline 136 & & 1.3581 & & 1.0729 & 0.9115 & 0.9592 \\
\hline 137 & & 1.2711 & & 1.0674 & 0.9090 & 0.9553 \\
\hline 138 & 1.1309 & 1.1484 & 1.0067 & 1.0641 & 0.9083 & 0.9577 \\
\hline 139 & 1.0934 & 1.1000 & 1.0089 & 1.0636 & 0.9132 & 0.9639 \\
\hline 140 & 1.0421 & 1.0171 & 1.0193 & 1.0626 & 0.9297 & 0.9852 \\
\hline 141 & 1.0158 & 0.9757 & 1.0171 & 1.0530 & 0.9355 & 0.9950 \\
\hline 142 & 0.9588 & 0.8947 & 0.9979 & 1.0155 & 0.9452 & 1.0133 \\
\hline 143 & 0.9352 & 0.8642 & 0.9904 & 0.9958 & 0.9585 & 1.0331 \\
\hline 144 & 0.8981 & 0.8187 & 0.9853 & 0.9615 & 1.0165 & 1.1088 \\
\hline 145 & 0.8858 & 0.8014 & 0.9837 & 0.9470 & 1.0644 & 1.1660 \\
\hline 146 & 0.8619 & 0.7756 & 0.9920 & 0.9169 & 1.2022 & 1.3334 \\
\hline 147 & & 1.5690 & & 1.1045 & 0.9361 & 0.9853 \\
\hline 148 & & 1.4316 & & 1.0861 & 0.9226 & 0.9707 \\
\hline 149 & 1.2422 & 1.2285 & 1.0027 & 1.0661 & 0.9056 & 0.9536 \\
\hline 150 & 1.1740 & 1.1569 & 0.9997 & 1.0599 & 0.9017 & 0.9516 \\
\hline 151 & 1.0857 & 1.0535 & 1.0040 & 1.0560 & 0.9072 & 0.9604 \\
\hline 152 & 1.0505 & 1.0108 & 1.0035 & 1.0498 & 0.9126 & 0.9686 \\
\hline 153 & 0.9963 & 0.9341 & 0.9973 & 1.0324 & 0.9239 & 0.9888 \\
\hline 154 & 0.9696 & 0.9012 & 0.9902 & 1.0164 & 0.9333 & 1.0031 \\
\hline 155 & 0.9232 & 0.8437 & 0.9758 & 0.9831 & 0.9683 & 1.0507 \\
\hline 156 & 0.9092 & 0.8250 & 0.9726 & 0.9680 & 1.0034 & 1.0952 \\
\hline 157 & 0.8892 & 0.7994 & 0.9778 & 0.9470 & 1.1156 & 1.2347 \\
\hline 158 & 0.8857 & 0.7937 & 0.9847 & 0.9414 & 1.1971 & 1.3296 \\
\hline 159 & 1.4391 & 1.3876 & 1.0159 & 1.0837 & 0.9176 & 0.9674 \\
\hline
\end{tabular}




\begin{tabular}{|c|c|c|c|c|c|c|}
\hline 160 & 1.3196 & 1.2724 & 1.0039 & 1.0666 & 0.9044 & 0.9552 \\
\hline 161 & 1.1628 & 1.1148 & 0.9946 & 1.0529 & 0.8960 & 0.9475 \\
\hline 162 & 1.1075 & 1.0585 & 0.9939 & 1.0482 & 0.8953 & 0.9498 \\
\hline 163 & 1.0373 & 0.9737 & 0.9943 & 1.0393 & 0.9058 & 0.9648 \\
\hline 164 & 1.0074 & 0.9373 & 0.9893 & 1.0300 & 0.9128 & 0.9761 \\
\hline 165 & 0.9535 & 0.8729 & 0.9732 & 1.0002 & 0.9321 & 1.0077 \\
\hline 166 & 0.9325 & 0.8475 & 0.9663 & 0.9848 & 0.9555 & 1.0371 \\
\hline 167 & 0.9021 & 0.8120 & 0.9587 & 0.9597 & 1.0340 & 1.1362 \\
\hline 168 & 0.8960 & 0.8037 & 0.9630 & 0.9536 & 1.0999 & 1.2186 \\
\hline 169 & 1.5724 & 1.4815 & 1.0279 & 1.0956 & 0.9281 & 0.9790 \\
\hline 170 & 1.2971 & 1.2209 & 0.9934 & 1.0541 & 0.8933 & 0.9438 \\
\hline 171 & 1.2051 & 1.1321 & 0.9843 & 1.0431 & 0.8849 & 0.9377 \\
\hline 172 & 1.0975 & 1.0206 & 0.9885 & 1.0393 & 0.8914 & 0.9466 \\
\hline 173 & 1.0563 & 0.9784 & 0.9868 & 1.0349 & 0.8962 & 0.9550 \\
\hline 174 & 0.9936 & 0.9074 & 0.9776 & 1.0158 & 0.9104 & 0.9777 \\
\hline 175 & 0.9646 & 0.8773 & 0.9685 & 1.0029 & 0.9222 & 0.9954 \\
\hline 176 & 0.9154 & 0.8239 & 0.9457 & 0.9676 & 0.9653 & 1.0532 \\
\hline 177 & 0.9028 & 0.8095 & 0.9435 & 0.9583 & 1.0109 & 1.1117 \\
\hline 178 & 0.8992 & 0.8019 & 0.9569 & 0.9545 & 1.1644 & 1.2962 \\
\hline 179 & 1.5280 & 1.4115 & 1.0064 & 1.0705 & 0.9079 & 0.9561 \\
\hline 180 & 1.3771 & 1.2735 & 0.9879 & 1.0475 & 0.8867 & 0.9366 \\
\hline 181 & 1.1793 & 1.0856 & 0.9759 & 1.0304 & 0.8765 & 0.9289 \\
\hline 182 & 1.1207 & 1.0282 & 0.9788 & 1.0302 & 0.8809 & 0.9353 \\
\hline 183 & 1.0455 & 0.9498 & 0.9797 & 1.0278 & 0.8956 & 0.9577 \\
\hline 184 & 1.0119 & 0.9155 & 0.9757 & 1.0189 & 0.9039 & 0.9692 \\
\hline 185 & 0.9484 & 0.8514 & 0.9505 & 0.9852 & 0.9221 & 0.9989 \\
\hline 186 & 0.9218 & 0.8242 & 0.9374 & 0.9661 & 0.9455 & 1.0307 \\
\hline 187 & 0.8889 & 0.7917 & 0.9234 & 0.9419 & 1.0455 & 1.1537 \\
\hline 188 & 0.8903 & 0.7913 & 0.9310 & 0.9441 & 1.1294 & 1.2540 \\
\hline 189 & 1.3115 & 1.1928 & 0.9564 & 1.0120 & 0.8568 & 0.9054 \\
\hline 190 & 1.1958 & 1.0859 & 0.9489 & 1.0012 & 0.8500 & 0.9007 \\
\hline 191 & 1.1091 & 0.9998 & 0.9782 & 1.0274 & 0.8819 & 0.9373 \\
\hline 192 & 1.0689 & 0.9613 & 0.9813 & 1.0285 & 0.8918 & 0.9509 \\
\hline 193 & 1.0017 & 0.8935 & 0.9684 & 1.0106 & 0.9075 & 0.9751 \\
\hline 194 & 0.9668 & 0.8608 & 0.9540 & 0.9919 & 0.9161 & 0.9903 \\
\hline 195 & 0.8874 & 0.7880 & 0.9010 & 0.9318 & 0.9391 & 1.0269 \\
\hline 196 & 0.8727 & 0.7731 & 0.8933 & 0.9200 & 0.9984 & 1.0980 \\
\hline 197 & 1.3436 & 1.2085 & 0.9113 & 0.9620 & 0.8176 & 0.8607 \\
\hline 198 & 1.1698 & 1.0454 & 0.9482 & 0.9979 & 0.8487 & 0.8995 \\
\hline 199 & 1.1367 & 1.0105 & 0.9706 & 1.0204 & 0.8715 & 0.9248 \\
\hline 200 & 1.0666 & 0.9446 & 0.9839 & 1.0295 & 0.8999 & 0.9595 \\
\hline 201 & 1.0312 & 0.9115 & 0.9774 & 1.0215 & 0.9083 & 0.9731 \\
\hline 202 & 0.9512 & 0.8389 & 0.9385 & 0.9772 & 0.9186 & 0.9949 \\
\hline 203 & 0.9026 & 0.7962 & 0.9033 & 0.9379 & 0.9234 & 1.0050 \\
\hline 204 & 0.8206 & 0.7237 & 0.8392 & 0.8667 & 1.0023 & 1.1069 \\
\hline
\end{tabular}




\begin{tabular}{|l|l|l|l|l|l|l|l|}
\hline 205 & 1.1493 & 1.0121 & 0.9023 & 0.9475 & & 0.8072 & 0.8497 \\
\hline 206 & 1.1344 & 0.9942 & 0.9820 & 1.0314 & & 0.8845 & 0.9380 \\
\hline 207 & 1.1026 & 0.9649 & 0.9940 & 1.0416 & & 0.9038 & 0.9605 \\
\hline 208 & 1.0313 & 0.9017 & 0.9805 & 1.0242 & & 0.9200 & 0.9874 \\
\hline 209 & 0.9821 & 0.8586 & 0.9541 & 0.9973 & & 0.9215 & 0.9928 \\
\hline 210 & 0.8518 & 0.7456 & 0.8514 & 0.8849 & & 0.8897 & 0.9714 \\
\hline
\end{tabular}

Table B3. Block-wide pin fission density values, 3-D stylized ARI core, axial layer 5 and 6

\begin{tabular}{|c|c|c|c|c|c|c|c|}
\hline$\#$ & 1 & 2 & 3 & 4 & 5 & 6 & 7 \\
\hline 1 & & 0.8250 & & 0.9178 & 0.9262 & 1.3002 & 0.8912 \\
\hline 2 & & 0.8814 & & 0.9997 & 1.0121 & 1.4087 & 0.9361 \\
\hline 3 & & 0.8888 & & 1.0115 & 1.0241 & 1.4162 & 0.9304 \\
\hline 4 & & 0.8724 & & 0.9966 & 1.0125 & 1.3652 & 0.8849 \\
\hline 5 & & 0.8527 & & 0.9670 & 0.9842 & 1.3081 & 0.8469 \\
\hline 6 & & 0.7697 & & 0.8588 & 0.8742 & 1.1289 & 0.7334 \\
\hline 7 & & 0.9164 & & 0.9249 & 0.9343 & 1.1678 & 0.9331 \\
\hline 8 & & 0.8974 & & 0.9644 & 0.9777 & 1.2705 & 0.9330 \\
\hline 9 & & 0.9033 & & 0.9897 & 1.0027 & 1.3054 & 0.9379 \\
\hline 10 & & 0.8968 & & 1.0047 & 1.0206 & 1.2983 & 0.9152 \\
\hline 11 & & 0.8859 & & 0.9982 & 1.0110 & 1.2733 & 0.8943 \\
\hline 12 & & 0.8473 & & 0.9502 & 0.9675 & 1.1786 & 0.8266 \\
\hline 13 & & 0.8159 & & 0.9129 & 0.9292 & 1.1008 & 0.7794 \\
\hline 14 & & 0.7701 & & 0.8376 & 0.8568 & 0.9361 & 0.7013 \\
\hline 15 & & 0.9824 & & 0.9746 & 0.9855 & 1.1925 & 0.9775 \\
\hline 16 & & 0.9509 & & 0.9690 & 0.9802 & 1.1928 & 0.9498 \\
\hline 17 & & 0.9364 & & 1.0012 & 1.0111 & 1.2340 & 0.9456 \\
\hline 18 & & 0.9246 & & 1.0052 & 1.0161 & 1.2228 & 0.9285 \\
\hline 19 & & 0.8891 & & 0.9883 & 1.0002 & 1.1686 & 0.8832 \\
\hline 20 & & 0.8703 & & 0.9691 & 0.9858 & 1.1218 & 0.8504 \\
\hline 21 & & 0.8212 & & 0.9067 & 0.9244 & 0.9895 & 0.7696 \\
\hline 22 & & 0.8170 & & 0.8913 & 0.9117 & 0.9366 & 0.7428 \\
\hline 23 & & 1.1153 & & 1.0237 & 1.0342 & 1.2073 & 1.0596 \\
\hline 24 & & 1.0659 & & 1.0073 & 1.0169 & 1.1873 & 1.0243 \\
\hline 25 & & 1.0172 & & 1.0033 & 1.0124 & 1.1782 & 0.9776 \\
\hline 26 & & 0.9865 & & 1.0047 & 1.0144 & 1.1723 & 0.9602 \\
\hline 27 & & 0.9398 & & 1.0082 & 1.0206 & 1.1528 & 0.9292 \\
\hline 28 & & 0.9173 & & 0.9982 & 1.0128 & 1.1221 & 0.9055 \\
\hline 29 & & 0.8730 & & 0.9639 & 0.9799 & 1.0356 & 0.8446 \\
\hline 30 & & 0.8580 & & 0.9420 & 0.9620 & 0.9844 & 0.8098 \\
\hline 31 & & 0.8449 & & 0.9156 & 0.9346 & 0.8878 & 0.7551 \\
\hline 32 & & 0.8561 & & 0.9116 & 0.9356 & 0.8380 & 0.7277 \\
\hline 33 & & 1.2115 & & 1.0440 & 1.0558 & 1.2086 & 1.0970 \\
\hline 34 & & & & 1.0192 & 1.0282 & 1.1626 & 1.0284 \\
\hline
\end{tabular}




\begin{tabular}{|c|c|c|c|c|c|c|c|}
\hline 35 & & & & 1.0167 & 1.0246 & 1.1490 & 1.0035 \\
\hline 36 & & 1.0335 & & 1.0203 & 1.0300 & 1.1338 & 0.9701 \\
\hline 37 & & 0.9901 & & 1.0172 & 1.0274 & 1.1154 & 0.9509 \\
\hline 38 & & 0.9256 & & 1.0006 & 1.0135 & 1.0596 & 0.9039 \\
\hline 39 & & 0.9020 & & 0.9853 & 0.9991 & 1.0179 & 0.8749 \\
\hline 40 & & 0.8676 & & 0.9465 & 0.9644 & 0.9228 & 0.8136 \\
\hline 41 & & 0.8615 & & 0.9376 & 0.9538 & 0.8776 & 0.7849 \\
\hline 42 & & 0.8723 & & 0.9216 & 0.9477 & 0.7790 & 0.7324 \\
\hline 43 & & & & 1.0407 & 1.0517 & 1.1760 & 1.0816 \\
\hline 44 & & & & 1.0319 & 1.0410 & 1.1517 & 1.0506 \\
\hline 45 & & & & 1.0306 & 1.0388 & 1.1267 & 1.0106 \\
\hline 46 & & & & 1.0308 & 1.0380 & 1.1122 & 0.9928 \\
\hline 47 & & 1.0043 & & 1.0249 & 1.0349 & 1.0741 & 0.9548 \\
\hline 48 & & 0.9619 & & 1.0191 & 1.0278 & 1.0445 & 0.9310 \\
\hline 49 & & 0.9069 & & 0.9844 & 0.9993 & 0.9657 & 0.8764 \\
\hline 50 & & 0.8885 & & 0.9720 & 0.9861 & 0.9224 & 0.8490 \\
\hline 51 & & 0.8704 & & 0.9376 & 0.9552 & 0.8338 & 0.7966 \\
\hline 52 & & 0.8707 & & 0.9271 & 0.9500 & 0.7870 & 0.7682 \\
\hline 53 & & 1.3309 & & 1.0497 & 1.0617 & 1.1843 & 1.1233 \\
\hline 54 & & & & 1.0445 & 1.0521 & 1.1634 & 1.0979 \\
\hline 55 & & & & 1.0383 & 1.0453 & 1.1298 & 1.0501 \\
\hline 56 & & & & 1.0379 & 1.0460 & 1.1129 & 1.0311 \\
\hline 57 & & & & 1.0426 & 1.0514 & 1.0864 & 1.0027 \\
\hline 58 & & 1.0597 & & 1.0429 & 1.0498 & 1.0665 & 0.9825 \\
\hline 59 & & 0.9672 & & 1.0239 & 1.0321 & 1.0060 & 0.9394 \\
\hline 60 & & 0.9364 & & 1.0088 & 1.0186 & 0.9697 & 0.9098 \\
\hline 61 & & 0.8950 & & 0.9707 & 0.9850 & 0.8844 & 0.8557 \\
\hline 62 & & 0.8807 & & 0.9532 & 0.9696 & 0.8404 & 0.8304 \\
\hline 63 & 1.0314 & 0.8731 & 1.1728 & 0.9245 & 0.9435 & 0.7572 & 0.7869 \\
\hline 64 & 1.0341 & 0.8759 & 1.2440 & 0.9129 & 0.9394 & 0.7212 & 0.7718 \\
\hline 65 & & 1.3830 & & 1.0305 & 1.0441 & 1.1589 & 1.1142 \\
\hline 66 & & & & 1.0403 & 1.0446 & 1.1332 & 1.0820 \\
\hline 67 & & & & 1.0424 & 1.0453 & 1.1211 & 1.0662 \\
\hline 68 & & & & 1.0512 & 1.0548 & 1.0951 & 1.0392 \\
\hline 69 & & & & 1.0536 & 1.0587 & 1.0803 & 1.0255 \\
\hline 70 & & 1.0547 & & 1.0594 & 1.0677 & 1.0502 & 1.0020 \\
\hline 71 & & 1.0065 & & 1.0499 & 1.0570 & 1.0194 & 0.9782 \\
\hline 72 & & 0.9359 & & 1.0109 & 1.0199 & 0.9363 & 0.9173 \\
\hline 73 & & 0.9114 & & 0.9897 & 1.0029 & 0.8944 & 0.8894 \\
\hline 74 & 1.0305 & 0.8813 & 1.0740 & 0.9489 & 0.9631 & 0.8149 & 0.8465 \\
\hline 75 & 1.0247 & 0.8724 & 1.1097 & 0.9277 & 0.9475 & 0.7783 & 0.8298 \\
\hline 76 & 1.0150 & 0.8620 & 1.2103 & 0.8893 & 0.9151 & 0.7149 & 0.8115 \\
\hline 77 & & 1.3305 & & 1.0175 & 1.0303 & 1.1277 & 1.0930 \\
\hline 78 & & & & 1.0319 & 1.0373 & 1.1233 & 1.0886 \\
\hline 79 & & & & 1.0464 & 1.0497 & 1.1013 & 1.0651 \\
\hline
\end{tabular}




\begin{tabular}{|c|c|c|c|c|c|c|c|}
\hline 80 & & & & 1.0570 & 1.0563 & 1.0914 & 1.0547 \\
\hline 81 & & & & 1.0733 & 1.0774 & 1.0751 & 1.0426 \\
\hline 82 & & 1.1093 & & 1.0835 & 1.0890 & 1.0658 & 1.0365 \\
\hline 83 & & 1.0106 & & 1.0653 & 1.0719 & 1.0022 & 0.9948 \\
\hline 84 & & 0.9660 & & 1.0376 & 1.0458 & 0.9541 & 0.9598 \\
\hline 85 & 1.0401 & 0.9098 & 1.0374 & 0.9871 & 0.9967 & 0.8693 & 0.9040 \\
\hline 86 & 1.0337 & 0.8911 & 1.0474 & 0.9627 & 0.9759 & 0.8315 & 0.8828 \\
\hline 87 & 1.0157 & 0.8654 & 1.0902 & 0.9148 & 0.9331 & 0.7620 & 0.8581 \\
\hline 88 & 1.0031 & 0.8508 & 1.1236 & 0.8878 & 0.9096 & 0.7370 & 0.8553 \\
\hline 89 & & 1.4094 & & 0.9389 & 0.9491 & 1.0534 & 1.0313 \\
\hline 90 & & 1.3558 & & 0.9915 & 1.0004 & 1.0976 & 1.0720 \\
\hline 91 & & & & 1.0328 & 1.0361 & 1.1020 & 1.0799 \\
\hline 92 & & & & 1.0458 & 1.0480 & 1.0973 & 1.0747 \\
\hline 93 & & & & 1.0647 & 1.0653 & 1.0803 & 1.0597 \\
\hline 94 & & & & 1.0815 & 1.0791 & 1.0762 & 1.0617 \\
\hline 95 & 1.0605 & 0.9592 & 1.0403 & 1.0344 & 1.0402 & 0.9278 & 0.9657 \\
\hline 96 & 1.0483 & 0.9255 & 1.0312 & 1.0041 & 1.0122 & 0.8861 & 0.9377 \\
\hline 97 & 1.0281 & 0.8868 & 1.0375 & 0.9551 & 0.9641 & 0.8150 & 0.9067 \\
\hline 98 & 1.0123 & 0.8685 & 1.0437 & 0.9247 & 0.9382 & 0.7830 & 0.8954 \\
\hline 99 & 0.9740 & 0.8273 & 1.0710 & 0.8571 & 0.8772 & 0.7295 & 0.8877 \\
\hline 100 & 0.9199 & 0.7821 & 1.0704 & 0.7974 & 0.8211 & 0.7039 & 0.8795 \\
\hline 101 & & 1.3144 & & 0.9934 & 0.9983 & 1.0796 & 1.0643 \\
\hline 102 & & & & 1.0262 & 1.0288 & 1.1005 & 1.0834 \\
\hline 103 & & & & 1.0529 & 1.0487 & 1.0857 & 1.0733 \\
\hline 104 & & 1.2723 & & 1.0634 & 1.0612 & 1.0781 & 1.0718 \\
\hline 105 & & 1.1302 & & 1.0962 & 1.0961 & 1.0701 & 1.0767 \\
\hline 106 & 1.0767 & 0.9828 & 1.0512 & 1.0600 & 1.0628 & 0.9492 & 1.0064 \\
\hline 107 & 1.0419 & 0.9178 & 1.0226 & 0.9951 & 1.0024 & 0.8668 & 0.9517 \\
\hline 108 & 1.0319 & 0.8961 & 1.0180 & 0.9678 & 0.9751 & 0.8339 & 0.9415 \\
\hline 109 & 1.0052 & 0.8603 & 1.0216 & 0.9129 & 0.9255 & 0.7814 & 0.9366 \\
\hline 110 & 0.9743 & 0.8295 & 1.0079 & 0.8698 & 0.8848 & 0.7527 & 0.9290 \\
\hline 111 & & 1.4821 & & 0.9467 & & 1.0563 & 1.0406 \\
\hline 112 & & 1.4101 & & 0.9995 & & 1.0909 & 1.0769 \\
\hline 113 & & 1.2611 & & 1.0360 & & 1.0945 & 1.0871 \\
\hline 114 & & 1.2071 & & 1.0507 & & 1.0880 & 1.0851 \\
\hline 115 & & 1.1374 & & 1.0686 & & 1.0650 & 1.0714 \\
\hline 116 & & 1.1138 & & 1.0836 & & 1.0585 & 1.0748 \\
\hline 117 & 1.0604 & 0.9588 & 1.0303 & 1.0394 & & 0.9202 & 1.0020 \\
\hline 118 & 1.0459 & 0.9276 & 1.0143 & 1.0085 & & 0.8811 & 0.9809 \\
\hline 119 & 1.0271 & 0.8905 & 1.0039 & 0.9606 & & 0.8260 & 0.9722 \\
\hline 120 & 1.0145 & 0.8722 & 0.9978 & 0.9324 & & 0.8028 & 0.9799 \\
\hline 121 & 0.9757 & 0.8293 & 0.9811 & 0.8616 & & 0.7790 & 1.0112 \\
\hline 122 & 0.9254 & 0.7867 & 0.9369 & 0.8043 & & 0.7680 & 1.0286 \\
\hline 123 & & 1.4284 & & 1.0302 & & 1.1149 & 1.1090 \\
\hline 124 & & 1.3288 & & 1.0434 & & 1.1065 & 1.1016 \\
\hline
\end{tabular}




\begin{tabular}{|c|c|c|c|c|c|c|}
\hline 125 & & 1.1896 & & 1.0566 & 1.0777 & 1.0845 \\
\hline 126 & & 1.1445 & & 1.0641 & 1.0652 & 1.0768 \\
\hline 127 & 1.0163 & 1.0835 & 1.0403 & 1.0837 & 1.0426 & 1.0713 \\
\hline 128 & 1.0449 & 1.0641 & 1.0532 & 1.0950 & 1.0326 & 1.0730 \\
\hline 129 & 1.0636 & 1.0032 & 1.0463 & 1.0765 & 0.9739 & 1.0482 \\
\hline 130 & 1.0567 & 0.9668 & 1.0260 & 1.0490 & 0.9321 & 1.0230 \\
\hline 131 & 1.0376 & 0.9153 & 0.9989 & 0.9975 & 0.8652 & 0.9989 \\
\hline 132 & 1.0302 & 0.8984 & 0.9895 & 0.9722 & 0.8437 & 1.0017 \\
\hline 133 & 1.0166 & 0.8716 & 0.9808 & 0.9280 & 0.8109 & 1.0378 \\
\hline 134 & 1.0087 & 0.8587 & 0.9729 & 0.9024 & 0.8137 & 1.0743 \\
\hline 135 & & 1.5506 & & 1.0514 & 1.1419 & 1.1381 \\
\hline 136 & & 1.3175 & & 1.0549 & 1.1081 & 1.1088 \\
\hline 137 & & 1.2302 & & 1.0550 & 1.0901 & 1.0947 \\
\hline 138 & 0.9694 & 1.1200 & 1.0200 & 1.0641 & 1.0503 & 1.0758 \\
\hline 139 & 0.9914 & 1.0809 & 1.0228 & 1.0691 & 1.0332 & 1.0674 \\
\hline 140 & 1.0335 & 1.0267 & 1.0351 & 1.0748 & 0.9987 & 1.0604 \\
\hline 141 & 1.0409 & 0.9997 & 1.0300 & 1.0654 & 0.9717 & 1.0474 \\
\hline 142 & 1.0388 & 0.9428 & 1.0040 & 1.0285 & 0.9066 & 1.0178 \\
\hline 143 & 1.0360 & 0.9202 & 0.9923 & 1.0061 & 0.8787 & 1.0176 \\
\hline 144 & 1.0297 & 0.8941 & 0.9787 & 0.9667 & 0.8354 & 1.0398 \\
\hline 145 & 1.0280 & 0.8848 & 0.9703 & 0.9477 & 0.8317 & 1.0700 \\
\hline 146 & 1.0223 & 0.8716 & 0.9646 & 0.9091 & 0.8493 & 1.1715 \\
\hline 147 & & 1.5421 & & 1.0724 & 1.1555 & 1.1546 \\
\hline 148 & & 1.4088 & & 1.0651 & 1.1247 & 1.1265 \\
\hline 149 & 0.9295 & 1.2104 & 1.0158 & 1.0560 & 1.0766 & 1.0885 \\
\hline 150 & 0.9489 & 1.1423 & 1.0130 & 1.0596 & 1.0536 & 1.0748 \\
\hline 151 & 0.9913 & 1.0555 & 1.0192 & 1.0636 & 1.0170 & 1.0596 \\
\hline 152 & 1.0073 & 1.0236 & 1.0194 & 1.0614 & 0.9952 & 1.0518 \\
\hline 153 & 1.0287 & 0.9695 & 1.0086 & 1.0457 & 0.9430 & 1.0368 \\
\hline 154 & 1.0314 & 0.9462 & 1.0001 & 1.0300 & 0.9146 & 1.0312 \\
\hline 155 & 1.0268 & 0.9086 & 0.9763 & 0.9902 & 0.8651 & 1.0377 \\
\hline 156 & 1.0268 & 0.8973 & 0.9676 & 0.9738 & 0.8498 & 1.0588 \\
\hline 157 & 1.0356 & 0.8880 & 0.9604 & 0.9467 & 0.8449 & 1.1433 \\
\hline 158 & 1.0424 & 0.8915 & 0.9629 & 0.9380 & 0.8656 & 1.2148 \\
\hline 159 & 0.8835 & 1.3807 & 1.0237 & 1.0671 & 1.1140 & 1.1228 \\
\hline 160 & 0.9092 & 1.2664 & 1.0142 & 1.0570 & 1.0839 & 1.0963 \\
\hline 161 & 0.9522 & 1.1156 & 1.0092 & 1.0539 & 1.0377 & 1.0675 \\
\hline 162 & 0.9726 & 1.0649 & 1.0103 & 1.0521 & 1.0169 & 1.0571 \\
\hline 163 & 1.0080 & 1.0008 & 1.0077 & 1.0513 & 0.9737 & 1.0469 \\
\hline 164 & 1.0169 & 0.9745 & 1.0003 & 1.0435 & 0.9472 & 1.0410 \\
\hline 165 & 1.0198 & 0.9290 & 0.9785 & 1.0119 & 0.8931 & 1.0339 \\
\hline 166 & 1.0201 & 0.9113 & 0.9691 & 0.9958 & 0.8712 & 1.0431 \\
\hline 167 & 1.0271 & 0.8912 & 0.9537 & 0.9649 & 0.8471 & 1.1032 \\
\hline 168 & 1.0363 & 0.8906 & 0.9485 & 0.9570 & 0.8508 & 1.1561 \\
\hline 169 & 0.8660 & 1.4863 & 1.0345 & 1.0752 & 1.1383 & 1.1414 \\
\hline
\end{tabular}




\begin{tabular}{|c|c|c|c|c|c|c|}
\hline 170 & 0.9162 & 1.2270 & 1.0053 & 1.0513 & 1.0630 & 1.0834 \\
\hline 171 & 0.9363 & 1.1420 & 0.9982 & 1.0441 & 1.0361 & 1.0624 \\
\hline 172 & 0.9785 & 1.0427 & 1.0007 & 1.0485 & 0.9973 & 1.0510 \\
\hline 173 & 0.9963 & 1.0068 & 1.0004 & 1.0456 & 0.9778 & 1.0448 \\
\hline 174 & 1.0124 & 0.9539 & 0.9903 & 1.0287 & 0.9282 & 1.0392 \\
\hline 175 & 1.0161 & 0.9322 & 0.9770 & 1.0155 & 0.9042 & 1.0392 \\
\hline 176 & 1.0092 & 0.8937 & 0.9479 & 0.9787 & 0.8547 & 1.0615 \\
\hline 177 & 1.0167 & 0.8890 & 0.9412 & 0.9652 & 0.8472 & 1.1007 \\
\hline 178 & 1.0434 & 0.8961 & 0.9444 & 0.9562 & 0.8644 & 1.2395 \\
\hline 179 & 0.8860 & 1.4296 & 1.0138 & 1.0562 & 1.1041 & 1.1139 \\
\hline 180 & 0.9095 & 1.2900 & 0.9969 & 1.0429 & 1.0649 & 1.0812 \\
\hline 181 & 0.9454 & 1.1059 & 0.9897 & 1.0327 & 1.0146 & 1.0493 \\
\hline 182 & 0.9696 & 1.0536 & 0.9908 & 1.0375 & 0.9976 & 1.0470 \\
\hline 183 & 1.0035 & 0.9902 & 0.9935 & 1.0391 & 0.9647 & 1.0437 \\
\hline 184 & 1.0120 & 0.9622 & 0.9893 & 1.0344 & 0.9363 & 1.0443 \\
\hline 185 & 1.0069 & 0.9125 & 0.9582 & 0.9990 & 0.8847 & 1.0425 \\
\hline 186 & 1.0019 & 0.8927 & 0.9425 & 0.9785 & 0.8597 & 1.0560 \\
\hline 187 & 1.0077 & 0.8742 & 0.9193 & 0.9486 & 0.8405 & 1.1413 \\
\hline 188 & 1.0235 & 0.8825 & 0.9246 & 0.9508 & 0.8504 & 1.2245 \\
\hline 189 & 0.9081 & 1.2170 & 0.9644 & 1.0088 & 1.0239 & 1.0392 \\
\hline 190 & 0.9252 & 1.1108 & 0.9609 & 1.0054 & 0.9965 & 1.0236 \\
\hline 191 & 0.9874 & 1.0343 & 0.9913 & 1.0377 & 0.9887 & 1.0444 \\
\hline 192 & 1.0056 & 1.0018 & 0.9957 & 1.0421 & 0.9724 & 1.0452 \\
\hline 193 & 1.0148 & 0.9473 & 0.9815 & 1.0255 & 0.9262 & 1.0421 \\
\hline 194 & 1.0087 & 0.9198 & 0.9640 & 1.0064 & 0.8972 & 1.0442 \\
\hline 195 & 0.9737 & 0.8596 & 0.9079 & 0.9429 & 0.8301 & 1.0506 \\
\hline 196 & 0.9778 & 0.8510 & 0.8945 & 0.9288 & 0.8216 & 1.1079 \\
\hline 197 & 0.8918 & 1.2404 & 0.9174 & 0.9628 & 0.9857 & 0.9975 \\
\hline 198 & 0.9524 & 1.0754 & 0.9611 & 1.0051 & 0.9856 & 1.0199 \\
\hline 199 & 0.9901 & 1.0470 & 0.9869 & 1.0320 & 0.9932 & 1.0406 \\
\hline 200 & 1.0258 & 0.9929 & 1.0024 & 1.0472 & 0.9679 & 1.0525 \\
\hline 201 & 1.0297 & 0.9649 & 0.9928 & 1.0388 & 0.9474 & 1.0520 \\
\hline 202 & 1.0078 & 0.9024 & 0.9481 & 0.9924 & 0.8795 & 1.0457 \\
\hline 203 & 0.9796 & 0.8649 & 0.9115 & 0.9537 & 0.8401 & 1.0425 \\
\hline 204 & 0.9320 & 0.8048 & 0.8422 & 0.8776 & 0.7845 & 1.1134 \\
\hline 205 & 0.9273 & 1.0440 & 0.9140 & 0.9562 & 0.9469 & 0.9727 \\
\hline 206 & 1.0237 & 1.0362 & 0.9981 & 1.0451 & 0.9972 & 1.0511 \\
\hline 207 & 1.0444 & 1.0130 & 1.0108 & 1.0586 & 0.9895 & 1.0619 \\
\hline 208 & 1.0464 & 0.9608 & 0.9946 & 1.0455 & 0.9429 & 1.0629 \\
\hline 209 & 1.0271 & 0.9218 & 0.9676 & 1.0148 & 0.9036 & 1.0522 \\
\hline 210 & 0.9343 & 0.8163 & 0.8604 & 0.9025 & 0.7919 & 1.0034 \\
\hline
\end{tabular}

Table B4. Core normalized pin fission density, 2-D stylized ARO core 


\begin{tabular}{|c|c|c|c|c|c|c|c|}
\hline \# & 1 & 2 & 3 & 4 & 5 & 6 & 7 \\
\hline 1 & & 1.3039 & & 0.7797 & 0.8011 & 1.1439 & 0.7506 \\
\hline 2 & & 1.2705 & & 0.8523 & 0.8736 & 1.3173 & 0.8414 \\
\hline 3 & & 1.2245 & & 0.8651 & 0.8852 & 1.3737 & 0.8719 \\
\hline 4 & & 1.1262 & & 0.8575 & 0.8761 & 1.4358 & 0.9128 \\
\hline 5 & & 1.0657 & & 0.8374 & 0.8540 & 1.4537 & 0.9314 \\
\hline 6 & & 0.9139 & & 0.7518 & 0.7657 & 1.4178 & 0.9356 \\
\hline 7 & & 1.5821 & & 0.7890 & 0.8128 & 0.9695 & 0.7491 \\
\hline 8 & & 1.3661 & & 0.8224 & 0.8437 & 1.1238 & 0.7948 \\
\hline 9 & & 1.3161 & & 0.8436 & 0.8645 & 1.1915 & 0.8267 \\
\hline 10 & & 1.2100 & & 0.8584 & 0.8779 & 1.2794 & 0.8756 \\
\hline 11 & & 1.1567 & & 0.8547 & 0.8735 & 1.3098 & 0.8970 \\
\hline 12 & & 1.0441 & & 0.8235 & 0.8402 & 1.3494 & 0.9408 \\
\hline 13 & & 0.9831 & & 0.7944 & 0.8100 & 1.3527 & 0.9665 \\
\hline 14 & & 0.8851 & & 0.7444 & 0.7574 & 1.4156 & 1.1105 \\
\hline 15 & & 1.5864 & & 0.8309 & 0.8543 & 0.9972 & 0.7925 \\
\hline 16 & & 1.4527 & & 0.8253 & 0.8471 & 1.0289 & 0.7938 \\
\hline 17 & & 1.3223 & & 0.8532 & 0.8732 & 1.1366 & 0.8421 \\
\hline 18 & & 1.2612 & & 0.8564 & 0.8758 & 1.1733 & 0.8632 \\
\hline 19 & & 1.1409 & & 0.8479 & 0.8660 & 1.2294 & 0.9065 \\
\hline 20 & & 1.0866 & & 0.8359 & 0.8534 & 1.2522 & 0.9334 \\
\hline 21 & & 0.9748 & & 0.7916 & 0.8073 & 1.2816 & 1.0008 \\
\hline 22 & & 0.9495 & & 0.7877 & 0.8023 & 1.3552 & 1.0945 \\
\hline 23 & & 1.8780 & & 0.8781 & 0.9040 & 0.9642 & 0.8295 \\
\hline 24 & & 1.7080 & & 0.8612 & 0.8849 & 0.9710 & 0.8173 \\
\hline 25 & & 1.4987 & & 0.8528 & 0.8736 & 1.0236 & 0.8236 \\
\hline 26 & & 1.4030 & & 0.8552 & 0.8752 & 1.0571 & 0.8373 \\
\hline 27 & & 1.2590 & & 0.8592 & 0.8779 & 1.1218 & 0.8766 \\
\hline 28 & & 1.1931 & & 0.8549 & 0.8731 & 1.1483 & 0.8982 \\
\hline 29 & & 1.0748 & & 0.8327 & 0.8501 & 1.1941 & 0.9529 \\
\hline 30 & & 1.0281 & & 0.8208 & 0.8374 & 1.2282 & 0.9996 \\
\hline 31 & & 0.9721 & & 0.8098 & 0.8253 & 1.3591 & 1.1657 \\
\hline 32 & & 0.9657 & & 0.8166 & 0.8318 & 1.4658 & 1.2921 \\
\hline 33 & & 1.9827 & & 0.9002 & 0.9255 & 0.9444 & 0.8482 \\
\hline 34 & & & & 0.8706 & 0.8919 & 0.9589 & 0.8282 \\
\hline 35 & & & & 0.8635 & 0.8841 & 0.9754 & 0.8300 \\
\hline 36 & & 1.4369 & & 0.8670 & 0.8862 & 1.0319 & 0.8555 \\
\hline 37 & & 1.3427 & & 0.8659 & 0.8843 & 1.0575 & 0.8716 \\
\hline 38 & & 1.1833 & & 0.8561 & 0.8739 & 1.1065 & 0.9148 \\
\hline 39 & & 1.1220 & & 0.8479 & 0.8656 & 1.1336 & 0.9451 \\
\hline 40 & & 1.0262 & & 0.8255 & 0.8427 & 1.2025 & 1.0354 \\
\hline 41 & & 0.9990 & & 0.8221 & 0.8387 & 1.2676 & 1.1135 \\
\hline 42 & & 0.9793 & & 0.8304 & 0.8459 & 1.4678 & 1.3513 \\
\hline 43 & & & & 0.8936 & 0.9157 & 0.9243 & 0.8420 \\
\hline 44 & & & & 0.8828 & 0.9030 & 0.9306 & 0.8364 \\
\hline
\end{tabular}




\begin{tabular}{|c|c|c|c|c|c|c|c|}
\hline 45 & & & & 0.8758 & 0.8949 & 0.9649 & 0.8443 \\
\hline 46 & & & & 0.8747 & 0.8932 & 0.9851 & 0.8550 \\
\hline 47 & & 1.3438 & & 0.8736 & 0.8912 & 1.0337 & 0.8892 \\
\hline 48 & & 1.2462 & & 0.8686 & 0.8857 & 1.0571 & 0.9108 \\
\hline 49 & & 1.1109 & & 0.8501 & 0.8670 & 1.1067 & 0.9676 \\
\hline 50 & & 1.0625 & & 0.8411 & 0.8582 & 1.1444 & 1.0133 \\
\hline 51 & & 0.9992 & & 0.8278 & 0.8438 & 1.2602 & 1.1522 \\
\hline 52 & & 0.9836 & & 0.8279 & 0.8440 & 1.3514 & 1.2583 \\
\hline 53 & & 2.0935 & & 0.9102 & 0.9321 & 0.8994 & 0.8516 \\
\hline 54 & & & & 0.8983 & 0.9185 & 0.8999 & 0.8439 \\
\hline 55 & & & & 0.8861 & 0.9042 & 0.9180 & 0.8414 \\
\hline 56 & & & & 0.8833 & 0.9013 & 0.9317 & 0.8464 \\
\hline 57 & & & & 0.8858 & 0.9033 & 0.9744 & 0.8726 \\
\hline 58 & & 1.4405 & & 0.8849 & 0.9021 & 0.9960 & 0.8893 \\
\hline 59 & & 1.2302 & & 0.8746 & 0.8916 & 1.0403 & 0.9314 \\
\hline 60 & & 1.1570 & & 0.8657 & 0.8821 & 1.0650 & 0.9593 \\
\hline 61 & & 1.0529 & & 0.8428 & 0.8591 & 1.1309 & 1.0398 \\
\hline 62 & & 1.0180 & & 0.8348 & 0.8511 & 1.1852 & 1.1030 \\
\hline 63 & 0.9092 & 0.9767 & 1.1887 & 0.8263 & 0.8426 & 1.3462 & 1.2892 \\
\hline 64 & 0.9097 & 0.9680 & 1.2851 & 0.8272 & 0.8430 & 1.4585 & 1.4123 \\
\hline 65 & & 2.1361 & & 0.8984 & 0.9181 & 0.8661 & 0.8376 \\
\hline 66 & & & & 0.8918 & 0.9092 & 0.8833 & 0.8380 \\
\hline 67 & & & & 0.8902 & 0.9070 & 0.8937 & 0.8418 \\
\hline 68 & & & & 0.8917 & 0.9077 & 0.9247 & 0.8581 \\
\hline 69 & & & & 0.8941 & 0.9102 & 0.9448 & 0.8724 \\
\hline 70 & & 1.4027 & & 0.9000 & 0.9165 & 0.9954 & 0.9144 \\
\hline 71 & & 1.2937 & & 0.8945 & 0.9111 & 1.0166 & 0.9355 \\
\hline 72 & & 1.1379 & & 0.8675 & 0.8841 & 1.0548 & 0.9808 \\
\hline 73 & & 1.0822 & & 0.8549 & 0.8714 & 1.0854 & 1.0170 \\
\hline 74 & 0.9198 & 1.0076 & 1.0503 & 0.8328 & 0.8490 & 1.1804 & 1.1294 \\
\hline 75 & 0.9096 & 0.9808 & 1.1034 & 0.8243 & 0.8406 & 1.2510 & 1.2093 \\
\hline 76 & 0.8927 & 0.9438 & 1.2523 & 0.8098 & 0.8254 & 1.4450 & 1.4270 \\
\hline 77 & & 1.9804 & & 0.8844 & 0.9005 & 0.8463 & 0.8245 \\
\hline 78 & & & & 0.8886 & 0.9038 & 0.8595 & 0.8319 \\
\hline 79 & & & & 0.8916 & 0.9069 & 0.8849 & 0.8451 \\
\hline 80 & & & & 0.8955 & 0.9105 & 0.9015 & 0.8566 \\
\hline 81 & & & & 0.9093 & 0.9245 & 0.9476 & 0.8932 \\
\hline 82 & & 1.5004 & & 0.9194 & 0.9350 & 0.9781 & 0.9209 \\
\hline 83 & & 1.2707 & & 0.9077 & 0.9235 & 1.0212 & 0.9642 \\
\hline 84 & & 1.1817 & & 0.8874 & 0.9035 & 1.0327 & 0.9792 \\
\hline 85 & 0.9487 & 1.0647 & 0.9784 & 0.8537 & 0.8688 & 1.0810 & 1.0391 \\
\hline 86 & 0.9305 & 1.0229 & 1.0040 & 0.8394 & 0.8550 & 1.1220 & 1.0875 \\
\hline 87 & 0.9014 & 0.9643 & 1.0842 & 0.8153 & 0.8308 & 1.2485 & 1.2320 \\
\hline 88 & 0.8845 & 0.9376 & 1.1400 & 0.8011 & 0.8164 & 1.3338 & 1.3257 \\
\hline 89 & & 2.1054 & & 0.8245 & 0.8378 & 0.7689 & 0.7633 \\
\hline
\end{tabular}




\begin{tabular}{|c|c|c|c|c|c|c|c|}
\hline 90 & & 1.9931 & & 0.8629 & 0.8766 & 0.8113 & 0.8018 \\
\hline 91 & & & & 0.8854 & 0.8988 & 0.8497 & 0.8302 \\
\hline 92 & & & & 0.8926 & 0.9065 & 0.8662 & 0.8422 \\
\hline 93 & & & & 0.9023 & 0.9161 & 0.8990 & 0.8668 \\
\hline 94 & & & & 0.9138 & 0.9278 & 0.9243 & 0.8890 \\
\hline 95 & 0.9968 & 1.1490 & 0.9535 & 0.8855 & 0.9002 & 1.0290 & 0.9975 \\
\hline 96 & 0.9672 & 1.0877 & 0.9568 & 0.8646 & 0.8796 & 1.0469 & 1.0209 \\
\hline 97 & 0.9266 & 1.0065 & 0.9941 & 0.8344 & 0.8489 & 1.1270 & 1.1140 \\
\hline 98 & 0.9056 & 0.9713 & 1.0184 & 0.8175 & 0.8317 & 1.1793 & 1.1739 \\
\hline 99 & 0.8580 & 0.9038 & 1.0847 & 0.7758 & 0.7901 & 1.3185 & 1.3311 \\
\hline 100 & 0.8085 & 0.8464 & 1.1045 & 0.7319 & 0.7458 & 1.3738 & 1.3936 \\
\hline 101 & & 1.8798 & & 0.8610 & 0.8714 & 0.8048 & 0.7999 \\
\hline 102 & & & & 0.8832 & 0.8945 & 0.8337 & 0.8249 \\
\hline 103 & & & & 0.8954 & 0.9075 & 0.8626 & 0.8472 \\
\hline 104 & & 1.8067 & & 0.9025 & 0.9146 & 0.8795 & 0.8615 \\
\hline 105 & & 1.5329 & & 0.9276 & 0.9406 & 0.9310 & 0.9093 \\
\hline 106 & 1.0285 & 1.1849 & 0.9514 & 0.9043 & 0.9184 & 1.0141 & 0.9964 \\
\hline 107 & 0.9628 & 1.0636 & 0.9492 & 0.8592 & 0.8734 & 1.0494 & 1.0405 \\
\hline 108 & 0.9391 & 1.0210 & 0.9590 & 0.8421 & 0.8553 & 1.0861 & 1.0827 \\
\hline 109 & 0.8976 & 0.9547 & 0.9953 & 0.8092 & 0.8225 & 1.1947 & 1.2067 \\
\hline 110 & 0.8623 & 0.9093 & 0.9989 & 0.7788 & 0.7916 & 1.2418 & 1.2609 \\
\hline 111 & & 2.1110 & & 0.8320 & & 0.7593 & 0.7651 \\
\hline 112 & & 1.9950 & & 0.8689 & & 0.8004 & 0.8033 \\
\hline 113 & & 1.7504 & & 0.8897 & & 0.8350 & 0.8316 \\
\hline 114 & & 1.6662 & & 0.8964 & & 0.8492 & 0.8442 \\
\hline 115 & & 1.5527 & & 0.9052 & & 0.8776 & 0.8685 \\
\hline 116 & & 1.4899 & & 0.9162 & & 0.9010 & 0.8912 \\
\hline 117 & 1.0113 & 1.1353 & 0.9333 & 0.8879 & & 1.0030 & 0.9991 \\
\hline 118 & 0.9785 & 1.0800 & 0.9298 & 0.8665 & & 1.0223 & 1.0223 \\
\hline 119 & 0.9344 & 1.0036 & 0.9462 & 0.8367 & & 1.1039 & 1.1156 \\
\hline 120 & 0.9120 & 0.9694 & 0.9549 & 0.8195 & & 1.1574 & 1.1761 \\
\hline 121 & 0.8629 & 0.9038 & 0.9723 & 0.7776 & & 1.2987 & 1.3345 \\
\hline 122 & 0.8134 & 0.8471 & 0.9437 & 0.7333 & & 1.3553 & 1.4010 \\
\hline 123 & & 1.9794 & & 0.8949 & & 0.8222 & 0.8275 \\
\hline 124 & & 1.8246 & & 0.8969 & & 0.8314 & 0.8349 \\
\hline 125 & & 1.6075 & & 0.8978 & & 0.8485 & 0.8487 \\
\hline 126 & & 1.5363 & & 0.9009 & & 0.8609 & 0.8603 \\
\hline 127 & 1.1486 & 1.4059 & 0.9004 & 0.9148 & & 0.8982 & 0.8975 \\
\hline 128 & 1.1287 & 1.3532 & 0.9177 & 0.9243 & & 0.9250 & 0.9255 \\
\hline 129 & 1.0681 & 1.2179 & 0.9271 & 0.9127 & & 0.9655 & 0.9684 \\
\hline 130 & 1.0285 & 1.1500 & 0.9195 & 0.8915 & & 0.9785 & 0.9835 \\
\hline 131 & 0.9694 & 1.0517 & 0.9170 & 0.8572 & & 1.0312 & 1.0429 \\
\hline 132 & 0.9475 & 1.0156 & 0.9207 & 0.8430 & & 1.0739 & 1.0915 \\
\hline 133 & 0.9130 & 0.9618 & 0.9413 & 0.8191 & & 1.2040 & 1.2360 \\
\hline 134 & 0.8951 & 0.9369 & 0.9497 & 0.8049 & & 1.2913 & 1.3327 \\
\hline
\end{tabular}




\begin{tabular}{|c|c|c|c|c|c|c|}
\hline 135 & & 2.1332 & & 0.9145 & 0.8319 & 0.8421 \\
\hline 136 & & 1.7766 & & 0.9031 & 0.8360 & 0.8430 \\
\hline 137 & & 1.6489 & & 0.8998 & 0.8403 & 0.8466 \\
\hline 138 & 1.2166 & 1.4663 & 0.8752 & 0.8996 & 0.8586 & 0.8634 \\
\hline 139 & 1.1700 & 1.3955 & 0.8826 & 0.9018 & 0.8732 & 0.8779 \\
\hline 140 & 1.1067 & 1.2749 & 0.9018 & 0.9072 & 0.9131 & 0.9203 \\
\hline 141 & 1.0744 & 1.2147 & 0.9053 & 0.9012 & 0.9329 & 0.9420 \\
\hline 142 & 1.0082 & 1.1044 & 0.8998 & 0.8738 & 0.9739 & 0.9867 \\
\hline 143 & 0.9819 & 1.0618 & 0.8995 & 0.8609 & 1.0060 & 1.0227 \\
\hline 144 & 0.9424 & 0.9993 & 0.9088 & 0.8388 & 1.1073 & 1.1344 \\
\hline 145 & 0.9283 & 0.9770 & 0.9169 & 0.8298 & 1.1792 & 1.2146 \\
\hline 146 & 0.9072 & 0.9435 & 0.9427 & 0.8142 & 1.3808 & 1.4349 \\
\hline 147 & & 2.0872 & & 0.9292 & 0.8458 & 0.8582 \\
\hline 148 & & 1.8874 & & 0.9143 & 0.8387 & 0.8502 \\
\hline 149 & 1.3557 & 1.5906 & 0.8664 & 0.8979 & 0.8382 & 0.8475 \\
\hline 150 & 1.2707 & 1.4837 & 0.8670 & 0.8940 & 0.8439 & 0.8531 \\
\hline 151 & 1.1603 & 1.3325 & 0.8786 & 0.8957 & 0.8700 & 0.8800 \\
\hline 152 & 1.1188 & 1.2699 & 0.8826 & 0.8942 & 0.8861 & 0.8977 \\
\hline 153 & 1.0513 & 1.1589 & 0.8867 & 0.8828 & 0.9253 & 0.9397 \\
\hline 154 & 1.0216 & 1.1123 & 0.8860 & 0.8734 & 0.9507 & 0.9676 \\
\hline 155 & 0.9693 & 1.0335 & 0.8847 & 0.8501 & 1.0231 & 1.0472 \\
\hline 156 & 0.9522 & 1.0070 & 0.8893 & 0.8420 & 1.0800 & 1.1098 \\
\hline 157 & 0.9324 & 0.9734 & 0.9091 & 0.8327 & 1.2488 & 1.2938 \\
\hline 158 & 0.9309 & 0.9672 & 0.9247 & 0.8330 & 1.3613 & 1.4168 \\
\hline 159 & 1.6004 & 1.8252 & 0.8719 & 0.9118 & 0.8372 & 0.8498 \\
\hline 160 & 1.4520 & 1.6555 & 0.8641 & 0.8981 & 0.8319 & 0.8444 \\
\hline 161 & 1.2581 & 1.4244 & 0.8636 & 0.8886 & 0.8406 & 0.8528 \\
\hline 162 & 1.1916 & 1.3410 & 0.8659 & 0.8863 & 0.8512 & 0.8641 \\
\hline 163 & 1.1032 & 1.2169 & 0.8740 & 0.8843 & 0.8841 & 0.8990 \\
\hline 164 & 1.0670 & 1.1649 & 0.8746 & 0.8789 & 0.9037 & 0.9209 \\
\hline 165 & 1.0037 & 1.0739 & 0.8703 & 0.8595 & 0.9556 & 0.9773 \\
\hline 166 & 0.9791 & 1.0393 & 0.8697 & 0.8500 & 0.9971 & 1.0224 \\
\hline 167 & 0.9454 & 0.9896 & 0.8768 & 0.8357 & 1.1232 & 1.1601 \\
\hline 168 & 0.9399 & 0.9790 & 0.8881 & 0.8355 & 1.2182 & 1.2643 \\
\hline 169 & 1.7632 & 1.9588 & 0.8800 & 0.9231 & 0.8424 & 0.8571 \\
\hline 170 & 1.4246 & 1.5834 & 0.8560 & 0.8876 & 0.8233 & 0.8376 \\
\hline 171 & 1.3111 & 1.4541 & 0.8522 & 0.8794 & 0.8243 & 0.8388 \\
\hline 172 & 1.1756 & 1.2893 & 0.8611 & 0.8805 & 0.8502 & 0.8658 \\
\hline 173 & 1.1260 & 1.2258 & 0.8634 & 0.8783 & 0.8659 & 0.8827 \\
\hline 174 & 1.0508 & 1.1246 & 0.8634 & 0.8676 & 0.9061 & 0.9265 \\
\hline 175 & 1.0184 & 1.0817 & 0.8601 & 0.8589 & 0.9343 & 0.9573 \\
\hline 176 & 0.9617 & 1.0083 & 0.8520 & 0.8354 & 1.0159 & 1.0461 \\
\hline 177 & 0.9472 & 0.9881 & 0.8560 & 0.8313 & 1.0872 & 1.1230 \\
\hline 178 & 0.9439 & 0.9766 & 0.8829 & 0.8380 & 1.3023 & 1.3560 \\
\hline 179 & 1.7087 & 1.8577 & 0.8628 & 0.9013 & 0.8247 & 0.8399 \\
\hline
\end{tabular}




\begin{tabular}{|c|c|c|c|c|c|c|}
\hline 180 & 1.5238 & 1.6603 & 0.8481 & 0.8813 & 0.8127 & 0.8278 \\
\hline 181 & 1.2804 & 1.3874 & 0.8442 & 0.8695 & 0.8191 & 0.8346 \\
\hline 182 & 1.2072 & 1.3021 & 0.8488 & 0.8707 & 0.8325 & 0.8493 \\
\hline 183 & 1.1135 & 1.1871 & 0.8589 & 0.8737 & 0.8701 & 0.8897 \\
\hline 184 & 1.0736 & 1.1372 & 0.8580 & 0.8684 & 0.8909 & 0.9122 \\
\hline 185 & 0.9990 & 1.0467 & 0.8444 & 0.8444 & 0.9408 & 0.9663 \\
\hline 186 & 0.9689 & 1.0098 & 0.8378 & 0.8318 & 0.9834 & 1.0130 \\
\hline 187 & 0.9331 & 0.9651 & 0.8388 & 0.8192 & 1.1365 & 1.1766 \\
\hline 188 & 0.9342 & 0.9629 & 0.8515 & 0.8255 & 1.2513 & 1.2997 \\
\hline 189 & 1.4456 & 1.5471 & 0.8220 & 0.8515 & 0.7875 & 0.8038 \\
\hline 190 & 1.3056 & 1.3941 & 0.8179 & 0.8439 & 0.7892 & 0.8057 \\
\hline 191 & 1.1925 & 1.2608 & 0.8491 & 0.8699 & 0.8379 & 0.8561 \\
\hline 192 & 1.1440 & 1.2038 & 0.8548 & 0.8726 & 0.8580 & 0.8773 \\
\hline 193 & 1.0618 & 1.1072 & 0.8515 & 0.8626 & 0.8992 & 0.9219 \\
\hline 194 & 1.0217 & 1.0612 & 0.8424 & 0.8491 & 0.9244 & 0.9497 \\
\hline 195 & 0.9333 & 0.9637 & 0.8058 & 0.8031 & 0.9854 & 1.0163 \\
\hline 196 & 0.9153 & 0.9435 & 0.8060 & 0.7981 & 1.0733 & 1.1093 \\
\hline 197 & 1.4933 & 1.5802 & 0.7814 & 0.8112 & 0.7458 & 0.7609 \\
\hline 198 & 1.2730 & 1.3330 & 0.8176 & 0.8421 & 0.7920 & 0.8083 \\
\hline 199 & 1.2256 & 1.2779 & 0.8400 & 0.8625 & 0.8233 & 0.8411 \\
\hline 200 & 1.1392 & 1.1789 & 0.8579 & 0.8760 & 0.8708 & 0.8916 \\
\hline 201 & 1.0957 & 1.1309 & 0.8558 & 0.8705 & 0.8921 & 0.9141 \\
\hline 202 & 1.0042 & 1.0316 & 0.8291 & 0.8374 & 0.9328 & 0.9591 \\
\hline 203 & 0.9506 & 0.9747 & 0.8028 & 0.8073 & 0.9565 & 0.9844 \\
\hline 204 & 0.8625 & 0.8833 & 0.7585 & 0.7542 & 1.0907 & 1.1283 \\
\hline 205 & 1.2541 & 1.2960 & 0.7769 & 0.7999 & 0.7486 & 0.7642 \\
\hline 206 & 1.2229 & 1.2540 & 0.8511 & 0.8726 & 0.8393 & 0.8579 \\
\hline 207 & 1.1809 & 1.2082 & 0.8640 & 0.8841 & 0.8693 & 0.8888 \\
\hline 208 & 1.0958 & 1.1168 & 0.8588 & 0.8743 & 0.9096 & 0.9313 \\
\hline 209 & 1.0387 & 1.0585 & 0.8395 & 0.8524 & 0.9271 & 0.9510 \\
\hline 210 & 0.8979 & 0.9136 & 0.7567 & 0.7638 & 0.9289 & 0.9554 \\
\hline
\end{tabular}

Table B5. Core normalized pin fission density, 2-D stylized SRI core

\begin{tabular}{|c|c|c|c|c|c|c|c|}
\hline$\#$ & 1 & 2 & 3 & 4 & 5 & 6 & 7 \\
\hline 1 & & 0.7677 & & 0.7935 & 0.7847 & 1.3448 & 0.8607 \\
\hline 2 & & 0.8266 & & 0.8850 & 0.8746 & 1.5569 & 0.9744 \\
\hline 3 & & 0.8375 & & 0.9070 & 0.8967 & 1.6276 & 1.0149 \\
\hline 4 & & 0.8311 & & 0.9144 & 0.9041 & 1.7080 & 1.0714 \\
\hline 5 & & 0.8163 & & 0.9003 & 0.8902 & 1.7324 & 1.0977 \\
\hline 6 & & 0.7442 & & 0.8213 & 0.8118 & 1.6964 & 1.1099 \\
\hline 7 & & 0.8429 & & 0.7807 & 0.7752 & 1.1307 & 0.8488 \\
\hline 8 & & 0.8336 & & 0.8374 & 0.8301 & 1.3213 & 0.9129 \\
\hline 9 & & 0.8439 & & 0.8687 & 0.8605 & 1.4051 & 0.9542 \\
\hline
\end{tabular}




\begin{tabular}{|c|c|c|c|c|c|}
\hline 10 & 0.8444 & 0.9014 & 0.8932 & 1.5165 & 1.0203 \\
\hline 11 & 0.8385 & 0.9052 & 0.8974 & 1.5557 & 1.0504 \\
\hline 12 & 0.8115 & 0.8870 & 0.8800 & 1.6102 & 1.1108 \\
\hline 13 & 0.7881 & 0.8627 & 0.8556 & 1.6160 & 1.1445 \\
\hline 14 & 0.7551 & 0.8214 & 0.8162 & 1.6972 & 1.3233 \\
\hline 15 & 0.9043 & 0.8255 & 0.8210 & 1.1643 & 0.8998 \\
\hline 16 & 0.8794 & 0.8317 & 0.8267 & 1.2058 & 0.9075 \\
\hline 17 & 0.8719 & 0.8797 & 0.8738 & 1.3402 & 0.9742 \\
\hline 18 & 0.8650 & 0.8924 & 0.8860 & 1.3877 & 1.0037 \\
\hline 19 & 0.8423 & 0.8996 & 0.8940 & 1.4613 & 1.0630 \\
\hline 20 & 0.8295 & 0.8942 & 0.8893 & 1.4916 & 1.0997 \\
\hline 21 & 0.7932 & 0.8609 & 0.8568 & 1.5327 & 1.1872 \\
\hline 22 & 0.7969 & 0.8637 & 0.8601 & 1.6235 & 1.3033 \\
\hline 23 & 1.0218 & 0.8512 & 0.8499 & 1.1171 & 0.9330 \\
\hline 24 & 0.9801 & 0.8463 & 0.8439 & 1.1294 & 0.9250 \\
\hline 25 & 0.9392 & 0.8615 & 0.8576 & 1.2011 & 0.9437 \\
\hline 26 & 0.9138 & 0.8744 & 0.8702 & 1.2439 & 0.9652 \\
\hline 27 & 0.8808 & 0.8971 & 0.8929 & 1.3281 & 1.0212 \\
\hline 28 & 0.8649 & 0.9005 & 0.8968 & 1.3625 & 1.0516 \\
\hline 29 & 0.8337 & 0.8924 & 0.8895 & 1.4236 & 1.1239 \\
\hline 30 & 0.8243 & 0.8878 & 0.8849 & 1.4670 & 1.1831 \\
\hline 31 & 0.8263 & 0.8901 & 0.8880 & 1.6290 & 1.3878 \\
\hline 32 & 0.8418 & 0.9047 & 0.9026 & 1.7595 & 1.5428 \\
\hline 33 & 1.1064 & 0.8626 & 0.8639 & 1.0886 & 0.9491 \\
\hline 34 & & 0.8587 & 0.8574 & 1.1167 & 0.9396 \\
\hline 35 & & 0.8640 & 0.8618 & 1.1403 & 0.9472 \\
\hline 36 & 0.9561 & 0.8884 & 0.8858 & 1.2154 & 0.9876 \\
\hline 37 & 0.9217 & 0.8964 & 0.8935 & 1.2488 & 1.0125 \\
\hline 38 & 0.8729 & 0.9038 & 0.9017 & 1.3138 & 1.0717 \\
\hline 39 & 0.8569 & 0.9032 & 0.9018 & 1.3496 & 1.1125 \\
\hline 40 & 0.8350 & 0.8949 & 0.8942 & 1.4374 & 1.2270 \\
\hline 41 & 0.8367 & 0.8981 & 0.8980 & 1.5184 & 1.3236 \\
\hline 42 & 0.8622 & 0.9218 & 0.9217 & 1.7632 & 1.6157 \\
\hline 43 & & 0.8607 & 0.8622 & 1.0662 & 0.9459 \\
\hline 44 & & 0.8624 & 0.8632 & 1.0790 & 0.9457 \\
\hline 45 & & 0.8791 & 0.8778 & 1.1286 & 0.9667 \\
\hline 46 & & 0.8883 & 0.8872 & 1.1571 & 0.9844 \\
\hline 47 & 0.9370 & 0.9067 & 0.9055 & 1.2223 & 1.0348 \\
\hline 48 & 0.9028 & 0.9108 & 0.9095 & 1.2529 & 1.0652 \\
\hline 49 & 0.8631 & 0.9078 & 0.9073 & 1.3182 & 1.1403 \\
\hline 50 & 0.8523 & 0.9061 & 0.9063 & 1.3666 & 1.1987 \\
\hline 51 & 0.8474 & 0.9067 & 0.9082 & 1.5090 & 1.3723 \\
\hline 52 & 0.8551 & 0.9138 & 0.9156 & 1.6220 & 1.5017 \\
\hline 53 & 1.2119 & 0.8542 & 0.8602 & 1.0291 & 0.9464 \\
\hline 54 & & 0.8563 & 0.8599 & 1.0348 & 0.9440 \\
\hline
\end{tabular}




\begin{tabular}{|c|c|c|c|c|c|c|c|}
\hline 55 & & & & 0.8694 & 0.8707 & 1.0656 & 0.9543 \\
\hline 56 & & & & 0.8783 & 0.8789 & 1.0866 & 0.9659 \\
\hline 57 & & & & 0.9030 & 0.9028 & 1.1456 & 1.0066 \\
\hline 58 & & 0.9830 & & 0.9123 & 0.9112 & 1.1746 & 1.0316 \\
\hline 59 & & 0.9085 & & 0.9199 & 0.9199 & 1.2349 & 1.0907 \\
\hline 60 & & 0.8854 & & 0.9186 & 0.9193 & 1.2666 & 1.1280 \\
\hline 61 & & 0.8570 & & 0.9102 & 0.9115 & 1.3514 & 1.2313 \\
\hline 62 & & 0.8523 & & 0.9093 & 0.9114 & 1.4184 & 1.3106 \\
\hline 63 & 0.8787 & 0.8582 & 1.3793 & 0.9142 & 0.9169 & 1.6172 & 1.5390 \\
\hline 64 & 0.8916 & 0.8681 & 1.4959 & 0.9216 & 0.9248 & 1.7529 & 1.6892 \\
\hline 65 & & 1.2567 & & 0.8329 & 0.8414 & 0.9868 & 0.9273 \\
\hline 66 & & & & 0.8541 & 0.8583 & 1.0166 & 0.9407 \\
\hline 67 & & & & 0.8654 & 0.8681 & 1.0338 & 0.9512 \\
\hline 68 & & & & 0.8906 & 0.8910 & 1.0794 & 0.9815 \\
\hline 69 & & & & 0.9040 & 0.9044 & 1.1073 & 1.0035 \\
\hline 70 & & 0.9803 & & 0.9306 & 0.9308 & 1.1739 & 1.0620 \\
\hline 71 & & 0.9413 & & 0.9345 & 0.9353 & 1.2042 & 1.0934 \\
\hline 72 & & 0.8869 & & 0.9237 & 0.9250 & 1.2554 & 1.1551 \\
\hline 73 & & 0.8698 & & 0.9181 & 0.9199 & 1.2941 & 1.2024 \\
\hline 74 & 0.8623 & 0.8540 & 1.2076 & 0.9097 & 0.9123 & 1.4136 & 1.3434 \\
\hline 75 & 0.8657 & 0.8520 & 1.2747 & 0.9073 & 0.9105 & 1.4999 & 1.4418 \\
\hline 76 & 0.8743 & 0.8546 & 1.4577 & 0.9039 & 0.9085 & 1.7377 & 1.7083 \\
\hline 77 & & 1.2106 & & 0.8255 & 0.8331 & 0.9651 & 0.9161 \\
\hline 78 & & & & 0.8428 & 0.8483 & 0.9854 & 0.9305 \\
\hline 79 & & & & 0.8713 & 0.8738 & 1.0246 & 0.9574 \\
\hline 80 & & & & 0.8872 & 0.8889 & 1.0481 & 0.9766 \\
\hline 81 & & & & 0.9225 & 0.9239 & 1.1114 & 1.0295 \\
\hline 82 & & 1.0266 & & 0.9433 & 0.9444 & 1.1512 & 1.0669 \\
\hline 83 & & 0.9458 & & 0.9518 & 0.9533 & 1.2098 & 1.1287 \\
\hline 84 & & 0.9103 & & 0.9393 & 0.9413 & 1.2269 & 1.1512 \\
\hline 85 & 0.8573 & 0.8698 & 1.1134 & 0.9190 & 0.9216 & 1.2900 & 1.2306 \\
\hline 86 & 0.8566 & 0.8581 & 1.1484 & 0.9111 & 0.9144 & 1.3422 & 1.2916 \\
\hline 87 & 0.8579 & 0.8467 & 1.2530 & 0.8992 & 0.9034 & 1.4981 & 1.4703 \\
\hline 88 & 0.8555 & 0.8399 & 1.3233 & 0.8902 & 0.8948 & 1.6030 & 1.5854 \\
\hline 89 & & 1.2737 & & 0.7488 & 0.7589 & 0.8688 & 0.8389 \\
\hline 90 & & 1.2277 & & 0.7973 & 0.8055 & 0.9219 & 0.8871 \\
\hline 91 & & & & 0.8449 & 0.8499 & 0.9753 & 0.9315 \\
\hline 92 & & & & 0.8640 & 0.8677 & 0.9992 & 0.9511 \\
\hline 93 & & & & 0.8974 & 0.8993 & 1.0467 & 0.9908 \\
\hline 94 & & & & 0.9195 & 0.9212 & 1.0801 & 1.0214 \\
\hline 95 & 0.8634 & 0.9043 & 1.0740 & 0.9402 & 0.9428 & 1.2231 & 1.1737 \\
\hline 96 & 0.8565 & 0.8802 & 1.0832 & 0.9255 & 0.9284 & 1.2481 & 1.2054 \\
\hline 97 & 0.8537 & 0.8556 & 1.1372 & 0.9085 & 0.9116 & 1.3488 & 1.3238 \\
\hline 98 & 0.8493 & 0.8438 & 1.1711 & 0.8966 & 0.9007 & 1.4137 & 1.3989 \\
\hline 99 & 0.8297 & 0.8175 & 1.2583 & 0.8639 & 0.8690 & 1.5853 & 1.5919 \\
\hline
\end{tabular}




\begin{tabular}{|c|c|c|c|c|c|c|c|}
\hline 100 & 0.7932 & 0.7792 & 1.2866 & 0.8201 & 0.8261 & 1.6526 & 1.6687 \\
\hline 101 & & 1.1916 & & 0.8007 & 0.8075 & 0.9151 & 0.8888 \\
\hline 102 & & 1.1585 & & 0.8343 & 0.8400 & 0.9524 & 0.9221 \\
\hline 103 & & & & 0.8711 & 0.8745 & 0.9957 & 0.9591 \\
\hline 104 & & & & 0.8901 & 0.8924 & 1.0207 & 0.9817 \\
\hline 105 & & 1.0410 & & 0.9374 & 0.9390 & 1.0891 & 1.0471 \\
\hline 106 & 0.8684 & 0.9252 & 1.0648 & 0.9545 & 0.9573 & 1.2035 & 1.1702 \\
\hline 107 & 0.8530 & 0.8746 & 1.0741 & 0.9223 & 0.9257 & 1.2515 & 1.2312 \\
\hline 108 & 0.8496 & 0.8601 & 1.0912 & 0.9115 & 0.9143 & 1.2980 & 1.2851 \\
\hline 109 & 0.8429 & 0.8390 & 1.1438 & 0.8898 & 0.8943 & 1.4329 & 1.4384 \\
\hline 110 & 0.8226 & 0.8151 & 1.1536 & 0.8620 & 0.8669 & 1.4909 & 1.5062 \\
\hline 111 & & 1.3351 & & 0.7500 & & 0.8537 & 0.8401 \\
\hline 112 & & 1.2744 & & 0.7978 & & 0.9055 & 0.8884 \\
\hline 113 & & 1.1453 & & 0.8446 & & 0.9549 & 0.9325 \\
\hline 114 & & 1.0998 & & 0.8638 & & 0.9769 & 0.9520 \\
\hline 115 & & 1.0431 & & 0.8963 & & 1.0195 & 0.9920 \\
\hline 116 & & 1.0239 & & 0.9183 & & 1.0503 & 1.0228 \\
\hline 117 & 0.8546 & 0.9039 & 1.0443 & 0.9398 & & 1.1911 & 1.1755 \\
\hline 118 & 0.8485 & 0.8811 & 1.0464 & 0.9248 & & 1.2167 & 1.2072 \\
\hline 119 & 0.8476 & 0.8569 & 1.0766 & 0.9081 & & 1.3198 & 1.3252 \\
\hline 120 & 0.8432 & 0.8451 & 1.0921 & 0.8962 & & 1.3868 & 1.3999 \\
\hline 121 & 0.8246 & 0.8185 & 1.1225 & 0.8636 & & 1.5603 & 1.5956 \\
\hline 122 & 0.7882 & 0.7806 & 1.0945 & 0.8200 & & 1.6291 & 1.6765 \\
\hline 123 & & 1.2886 & & 0.8259 & & 0.9300 & 0.9177 \\
\hline 124 & & 1.2020 & & 0.8424 & & 0.9467 & 0.9322 \\
\hline 125 & & 1.0824 & & 0.8704 & & 0.9770 & 0.9596 \\
\hline 126 & & 1.0457 & & 0.8852 & & 0.9960 & 0.9790 \\
\hline 127 & 0.7967 & 0.9972 & 0.9708 & 0.9211 & & 1.0487 & 1.0329 \\
\hline 128 & 0.8230 & 0.9835 & 0.9973 & 0.9420 & & 1.0846 & 1.0706 \\
\hline 129 & 0.8482 & 0.9378 & 1.0231 & 0.9509 & & 1.1403 & 1.1320 \\
\hline 130 & 0.8459 & 0.9086 & 1.0218 & 0.9381 & & 1.1595 & 1.1542 \\
\hline 131 & 0.8424 & 0.8713 & 1.0313 & 0.9180 & & 1.2285 & 1.2336 \\
\hline 132 & 0.8436 & 0.8607 & 1.0422 & 0.9106 & & 1.2832 & 1.2946 \\
\hline 133 & 0.8463 & 0.8492 & 1.0772 & 0.8989 & & 1.4438 & 1.4730 \\
\hline 134 & 0.8446 & 0.8425 & 1.0924 & 0.8895 & & 1.5509 & 1.5908 \\
\hline 135 & & 1.3971 & & 0.8340 & & 0.9365 & 0.9297 \\
\hline 136 & & 1.1911 & & 0.8535 & & 0.9525 & 0.9432 \\
\hline 137 & & 1.1199 & & 0.8641 & & 0.9634 & 0.9538 \\
\hline 138 & 0.7520 & 1.0243 & 0.9287 & 0.8885 & & 0.9947 & 0.9854 \\
\hline 139 & 0.7722 & 0.9931 & 0.9449 & 0.9015 & & 1.0163 & 1.0076 \\
\hline 140 & 0.8122 & 0.9516 & 0.9800 & 0.9282 & & 1.0716 & 1.0675 \\
\hline 141 & 0.8248 & 0.9300 & 0.9917 & 0.9328 & & 1.0991 & 1.0982 \\
\hline 142 & 0.8322 & 0.8877 & 0.9999 & 0.9222 & & 1.1549 & 1.1602 \\
\hline 143 & 0.8354 & 0.8736 & 1.0058 & 0.9170 & & 1.1968 & 1.2074 \\
\hline 144 & 0.8430 & 0.8585 & 1.0290 & 0.9086 & & 1.3234 & 1.3480 \\
\hline
\end{tabular}




\begin{tabular}{|c|c|c|c|c|c|c|}
\hline 145 & 0.8482 & 0.8557 & 1.0440 & 0.9063 & 1.4126 & 1.4456 \\
\hline 146 & 0.8586 & 0.8575 & 1.0844 & 0.9028 & 1.6580 & 1.7146 \\
\hline 147 & & 1.3903 & & 0.8539 & 0.9540 & 0.9500 \\
\hline 148 & & 1.2711 & & 0.8550 & 0.9514 & 0.9480 \\
\hline 149 & 0.7128 & 1.0995 & 0.9017 & 0.8670 & 0.9623 & 0.9581 \\
\hline 150 & 0.7325 & 1.0418 & 0.9120 & 0.8760 & 0.9744 & 0.9711 \\
\hline 151 & 0.7719 & 0.9711 & 0.9405 & 0.9005 & 1.0139 & 1.0126 \\
\hline 152 & 0.7895 & 0.9454 & 0.9527 & 0.9093 & 1.0373 & 1.0383 \\
\hline 153 & 0.8133 & 0.9044 & 0.9718 & 0.9174 & 1.0911 & 1.0975 \\
\hline 154 & 0.8207 & 0.8884 & 0.9783 & 0.9168 & 1.1251 & 1.1351 \\
\hline 155 & 0.8294 & 0.8636 & 0.9899 & 0.9086 & 1.2176 & 1.2377 \\
\hline 156 & 0.8367 & 0.8592 & 1.0013 & 0.9078 & 1.2887 & 1.3161 \\
\hline 157 & 0.8560 & 0.8635 & 1.0358 & 0.9124 & 1.4956 & 1.5415 \\
\hline 158 & 0.8694 & 0.8723 & 1.0583 & 0.9196 & 1.6345 & 1.6918 \\
\hline 159 & 0.6734 & 1.2471 & 0.8897 & 0.8585 & 0.9506 & 0.9505 \\
\hline 160 & 0.6961 & 1.1476 & 0.8913 & 0.8598 & 0.9508 & 0.9510 \\
\hline 161 & 0.7354 & 1.0187 & 0.9084 & 0.8751 & 0.9719 & 0.9733 \\
\hline 162 & 0.7537 & 0.9769 & 0.9195 & 0.8850 & 0.9886 & 0.9917 \\
\hline 163 & 0.7872 & 0.9251 & 0.9433 & 0.9036 & 1.0359 & 1.0425 \\
\hline 164 & 0.7994 & 0.9044 & 0.9515 & 0.9076 & 1.0636 & 1.0726 \\
\hline 165 & 0.8128 & 0.8714 & 0.9608 & 0.9049 & 1.1323 & 1.1490 \\
\hline 166 & 0.8196 & 0.8620 & 0.9671 & 0.9039 & 1.1851 & 1.2063 \\
\hline 167 & 0.8358 & 0.8554 & 0.9880 & 0.9046 & 1.3414 & 1.3773 \\
\hline 168 & 0.8505 & 0.8625 & 1.0069 & 0.9118 & 1.4581 & 1.5045 \\
\hline 169 & 0.6570 & 1.3402 & 0.8885 & 0.8605 & 0.9525 & 0.9547 \\
\hline 170 & 0.6995 & 1.1144 & 0.8832 & 0.8551 & 0.9432 & 0.9463 \\
\hline 171 & 0.7186 & 1.0403 & 0.8886 & 0.8598 & 0.9499 & 0.9542 \\
\hline 172 & 0.7583 & 0.9567 & 0.9154 & 0.8835 & 0.9892 & 0.9964 \\
\hline 173 & 0.7741 & 0.9276 & 0.9255 & 0.8921 & 1.0122 & 1.0211 \\
\hline 174 & 0.7965 & 0.8874 & 0.9393 & 0.8999 & 1.0676 & 1.0816 \\
\hline 175 & 0.8037 & 0.8719 & 0.9439 & 0.8992 & 1.1049 & 1.1230 \\
\hline 176 & 0.8116 & 0.8474 & 0.9480 & 0.8916 & 1.2090 & 1.2362 \\
\hline 177 & 0.8228 & 0.8476 & 0.9592 & 0.8955 & 1.2975 & 1.3318 \\
\hline 178 & 0.8593 & 0.8696 & 1.0027 & 0.9185 & 1.5604 & 1.6151 \\
\hline 179 & 0.6715 & 1.2887 & 0.8714 & 0.8469 & 0.9346 & 0.9391 \\
\hline 180 & 0.6912 & 1.1674 & 0.8664 & 0.8418 & 0.9268 & 0.9320 \\
\hline 181 & 0.7255 & 1.0087 & 0.8816 & 0.8555 & 0.9450 & 0.9521 \\
\hline 182 & 0.7460 & 0.9640 & 0.8948 & 0.8681 & 0.9659 & 0.9743 \\
\hline 183 & 0.7801 & 0.9133 & 0.9215 & 0.8912 & 1.0192 & 1.0309 \\
\hline 184 & 0.7905 & 0.8927 & 0.9275 & 0.8951 & 1.0474 & 1.0623 \\
\hline 185 & 0.7968 & 0.8552 & 0.9266 & 0.8875 & 1.1135 & 1.1360 \\
\hline 186 & 0.7999 & 0.8416 & 0.9263 & 0.8832 & 1.1681 & 1.1950 \\
\hline 187 & 0.8174 & 0.8381 & 0.9413 & 0.8857 & 1.3566 & 1.3974 \\
\hline 188 & 0.8375 & 0.8518 & 0.9617 & 0.8997 & 1.4968 & 1.5472 \\
\hline 189 & 0.6911 & 1.1018 & 0.8407 & 0.8194 & 0.9004 & 0.9076 \\
\hline
\end{tabular}




\begin{tabular}{|l|l|l|l|l|l|l|l|}
\hline 190 & 0.7056 & 1.0108 & 0.8468 & 0.8252 & & 0.9080 & 0.9164 \\
\hline 191 & 0.7595 & 0.9475 & 0.8963 & 0.8724 & & 0.9739 & 0.9854 \\
\hline 192 & 0.7766 & 0.9221 & 0.9099 & 0.8848 & & 1.0023 & 1.0150 \\
\hline 193 & 0.7939 & 0.8808 & 0.9207 & 0.8930 & & 1.0583 & 1.0762 \\
\hline 194 & 0.7954 & 0.8605 & 0.9184 & 0.8878 & & 1.0922 & 1.1128 \\
\hline 195 & 0.7778 & 0.8128 & 0.8918 & 0.8552 & & 1.1711 & 1.2008 \\
\hline 196 & 0.7872 & 0.8123 & 0.8989 & 0.8583 & & 1.2793 & 1.3149 \\
\hline 197 & 0.6748 & 1.1207 & 0.7918 & 0.7738 & & 0.8491 & 0.8560 \\
\hline 198 & 0.7257 & 0.9793 & 0.8480 & 0.8291 & & 0.9128 & 0.9229 \\
\hline 199 & 0.7574 & 0.9560 & 0.8796 & 0.8599 & & 0.9542 & 0.9659 \\
\hline 200 & 0.7919 & 0.9146 & 0.9143 & 0.8927 & & 1.0192 & 1.0335 \\
\hline 201 & 0.7995 & 0.8933 & 0.9191 & 0.8959 & & 1.0477 & 1.0648 \\
\hline 202 & 0.7914 & 0.8460 & 0.9041 & 0.8785 & & 1.1039 & 1.1262 \\
\hline 203 & 0.7750 & 0.8147 & 0.8815 & 0.8549 & & 1.1345 & 1.1610 \\
\hline 204 & 0.7505 & 0.7702 & 0.8476 & 0.8146 & & 1.3016 & 1.3390 \\
\hline 205 & 0.7034 & 0.9503 & 0.7996 & 0.7843 & & 0.8609 & 0.8704 \\
\hline 206 & 0.7839 & 0.9487 & 0.8914 & 0.8745 & & 0.9734 & 0.9870 \\
\hline 207 & 0.8028 & 0.9310 & 0.9141 & 0.8959 & & 1.0135 & 1.0285 \\
\hline 208 & 0.8128 & 0.8913 & 0.9230 & 0.9038 & & 1.0692 & 1.0875 \\
\hline 209 & 0.8026 & 0.8613 & 0.9094 & 0.8897 & & 1.0942 & 1.1151 \\
\hline 210 & 0.7384 & 0.7703 & 0.8318 & 0.8115 & & 1.1030 & 1.1281 \\
\hline
\end{tabular}

Table B6. Core normalized pin fission density, 2-D stylized ARI core

\begin{tabular}{|c|c|c|c|c|c|c|c|}
\hline$\#$ & 1 & 2 & 3 & 4 & 5 & 6 & 7 \\
\hline 1 & & 1.0674 & & 0.9325 & 0.9788 & 1.0438 & 0.7675 \\
\hline 2 & & 1.1395 & & 1.0168 & 1.0693 & 1.1330 & 0.8081 \\
\hline 3 & & 1.1484 & & 1.0295 & 1.0843 & 1.1375 & 0.8028 \\
\hline 4 & & 1.1278 & & 1.0134 & 1.0692 & 1.0962 & 0.7652 \\
\hline 5 & & 1.0998 & & 0.9838 & 1.0392 & 1.0509 & 0.7310 \\
\hline 6 & & 0.9903 & & 0.8732 & 0.9246 & 0.9082 & 0.6335 \\
\hline 7 & & 1.1838 & & 0.9412 & 0.9876 & 0.9397 & 0.8066 \\
\hline 8 & & 1.1606 & & 0.9830 & 1.0328 & 1.0198 & 0.8060 \\
\hline 9 & & 1.1693 & & 1.0082 & 1.0596 & 1.0472 & 0.8109 \\
\hline 10 & & 1.1582 & & 1.0215 & 1.0758 & 1.0442 & 0.7924 \\
\hline 11 & & 1.1439 & & 1.0134 & 1.0680 & 1.0229 & 0.7726 \\
\hline 12 & & 1.0927 & & 0.9669 & 1.0218 & 0.9450 & 0.7141 \\
\hline 13 & & 1.0543 & & 0.9268 & 0.9811 & 0.8839 & 0.6741 \\
\hline 14 & & 0.9937 & & 0.8507 & 0.9055 & 0.7522 & 0.6053 \\
\hline 15 & & 1.2704 & & 0.9919 & 1.0413 & 0.9581 & 0.8428 \\
\hline 16 & & 1.2303 & & 0.9871 & 1.0359 & 0.9572 & 0.8204 \\
\hline 17 & & 1.2087 & & 1.0192 & 1.0712 & 0.9881 & 0.8150 \\
\hline 18 & & 1.1932 & & 1.0212 & 1.0747 & 0.9817 & 0.8024 \\
\hline 19 & & 1.1483 & & 1.0047 & 1.0592 & 0.9384 & 0.7626 \\
\hline
\end{tabular}




\begin{tabular}{|c|c|c|c|c|c|c|c|}
\hline 20 & & 1.1238 & & 0.9858 & 1.0403 & 0.9026 & 0.7353 \\
\hline 21 & & 1.0595 & & 0.9208 & 0.9759 & 0.7961 & 0.6649 \\
\hline 22 & & 1.0553 & & 0.9071 & 0.9632 & 0.7546 & 0.6415 \\
\hline 23 & & 1.4440 & & 1.0424 & 1.0945 & 0.9702 & 0.9148 \\
\hline 24 & & 1.3807 & & 1.0261 & 1.0761 & 0.9526 & 0.8828 \\
\hline 25 & & 1.3158 & & 1.0202 & 1.0697 & 0.9448 & 0.8430 \\
\hline 26 & & 1.2737 & & 1.0230 & 1.0734 & 0.9421 & 0.8294 \\
\hline 27 & & 1.2152 & & 1.0242 & 1.0776 & 0.9243 & 0.8014 \\
\hline 28 & & 1.1863 & & 1.0162 & 1.0701 & 0.9022 & 0.7812 \\
\hline 29 & & 1.1288 & & 0.9805 & 1.0352 & 0.8333 & 0.7287 \\
\hline 30 & & 1.1069 & & 0.9601 & 1.0156 & 0.7919 & 0.7007 \\
\hline 31 & & 1.0914 & & 0.9293 & 0.9872 & 0.7118 & 0.6522 \\
\hline 32 & & 1.1023 & & 0.9260 & 0.9874 & 0.6715 & 0.6283 \\
\hline 33 & & 1.5668 & & 1.0647 & 1.1186 & 0.9674 & 0.9466 \\
\hline 34 & & & & 1.0387 & 1.0890 & 0.9342 & 0.8870 \\
\hline 35 & & & & 1.0335 & 1.0830 & 0.9221 & 0.8656 \\
\hline 36 & & 1.3347 & & 1.0376 & 1.0880 & 0.9103 & 0.8372 \\
\hline 37 & & 1.2801 & & 1.0344 & 1.0859 & 0.8951 & 0.8200 \\
\hline 38 & & 1.1967 & & 1.0174 & 1.0700 & 0.8501 & 0.7796 \\
\hline 39 & & 1.1662 & & 1.0026 & 1.0566 & 0.8193 & 0.7560 \\
\hline 40 & & 1.1198 & & 0.9631 & 1.0188 & 0.7428 & 0.7030 \\
\hline 41 & & 1.1126 & & 0.9500 & 1.0076 & 0.7055 & 0.6788 \\
\hline 42 & & 1.1268 & & 0.9384 & 1.0010 & 0.6240 & 0.6324 \\
\hline 43 & & & & 1.0594 & 1.1113 & 0.9415 & 0.9342 \\
\hline 44 & & & & 1.0513 & 1.1013 & 0.9247 & 0.9094 \\
\hline 45 & & & & 1.0490 & 1.0977 & 0.9037 & 0.8725 \\
\hline 46 & & & & 1.0480 & 1.0970 & 0.8917 & 0.8562 \\
\hline 47 & & 1.2997 & & 1.0439 & 1.0950 & 0.8619 & 0.8248 \\
\hline 48 & & 1.2441 & & 1.0352 & 1.0866 & 0.8385 & 0.8044 \\
\hline 49 & & 1.1728 & & 1.0037 & 1.0568 & 0.7768 & 0.7576 \\
\hline 50 & & 1.1494 & & 0.9869 & 1.0414 & 0.7430 & 0.7334 \\
\hline 51 & & 1.1244 & & 0.9538 & 1.0117 & 0.6700 & 0.6871 \\
\hline 52 & & 1.1255 & & 0.9434 & 1.0042 & 0.6313 & 0.6637 \\
\hline 53 & & 1.7237 & & 1.0684 & 1.1241 & 0.9496 & 0.9691 \\
\hline 54 & & & & 1.0619 & 1.1139 & 0.9321 & 0.9463 \\
\hline 55 & & & & 1.0572 & 1.1062 & 0.9058 & 0.9078 \\
\hline 56 & & & & 1.0574 & 1.1053 & 0.8935 & 0.8910 \\
\hline 57 & & & & 1.0626 & 1.1112 & 0.8720 & 0.8645 \\
\hline 58 & & 1.3715 & & 1.0598 & 1.1092 & 0.8555 & 0.8486 \\
\hline 59 & & 1.2501 & & 1.0425 & 1.0926 & 0.8082 & 0.8098 \\
\hline 60 & & 1.2089 & & 1.0269 & 1.0782 & 0.7786 & 0.7872 \\
\hline 61 & & 1.1537 & & 0.9864 & 1.0409 & 0.7102 & 0.7401 \\
\hline 62 & & 1.1381 & & 0.9690 & 1.0248 & 0.6762 & 0.7182 \\
\hline 63 & 1.0742 & 1.1270 & 1.1887 & 0.9385 & 0.9985 & 0.6083 & 0.6803 \\
\hline 64 & 1.0796 & 1.1302 & 1.2615 & 0.9275 & 0.9912 & 0.5779 & 0.6659 \\
\hline
\end{tabular}




\begin{tabular}{|c|c|c|c|c|c|c|c|}
\hline 65 & & 1.7934 & & 1.0502 & 1.1047 & 0.9282 & 0.9622 \\
\hline 66 & & & & 1.0575 & 1.1070 & 0.9096 & 0.9346 \\
\hline 67 & & & & 1.0613 & 1.1084 & 0.8988 & 0.9207 \\
\hline 68 & & & & 1.0688 & 1.1155 & 0.8789 & 0.8953 \\
\hline 69 & & & & 1.0732 & 1.1200 & 0.8678 & 0.8839 \\
\hline 70 & & 1.3640 & & 1.0787 & 1.1264 & 0.8416 & 0.8615 \\
\hline 71 & & 1.3003 & & 1.0685 & 1.1180 & 0.8166 & 0.8421 \\
\hline 72 & & 1.2090 & & 1.0284 & 1.0788 & 0.7517 & 0.7934 \\
\hline 73 & & 1.1776 & & 1.0065 & 1.0583 & 0.7186 & 0.7710 \\
\hline 74 & 1.0730 & 1.1385 & 1.0897 & 0.9646 & 1.0192 & 0.6540 & 0.7315 \\
\hline 75 & 1.0682 & 1.1257 & 1.1244 & 0.9446 & 1.0014 & 0.6247 & 0.7172 \\
\hline 76 & 1.0590 & 1.1101 & 1.2287 & 0.9040 & 0.9664 & 0.5735 & 0.7028 \\
\hline 77 & & 1.7255 & & 1.0390 & 1.0884 & 0.9033 & 0.9434 \\
\hline 78 & & & & 1.0511 & 1.0987 & 0.8996 & 0.9376 \\
\hline 79 & & & & 1.0655 & 1.1105 & 0.8838 & 0.9186 \\
\hline 80 & & & & 1.0732 & 1.1180 & 0.8754 & 0.9097 \\
\hline 81 & & & & 1.0924 & 1.1386 & 0.8605 & 0.8975 \\
\hline 82 & & 1.4352 & & 1.1035 & 1.1502 & 0.8535 & 0.8935 \\
\hline 83 & & 1.3045 & & 1.0843 & 1.1327 & 0.8037 & 0.8573 \\
\hline 84 & & 1.2471 & & 1.0553 & 1.1047 & 0.7660 & 0.8280 \\
\hline 85 & 1.0845 & 1.1746 & 1.0510 & 1.0028 & 1.0534 & 0.6971 & 0.7814 \\
\hline 86 & 1.0755 & 1.1498 & 1.0607 & 0.9785 & 1.0300 & 0.6668 & 0.7636 \\
\hline 87 & 1.0588 & 1.1164 & 1.1054 & 0.9315 & 0.9867 & 0.6128 & 0.7413 \\
\hline 88 & 1.0463 & 1.0986 & 1.1402 & 0.9037 & 0.9614 & 0.5929 & 0.7392 \\
\hline 89 & & 1.8270 & & 0.9568 & 1.0050 & 0.8446 & 0.8906 \\
\hline 90 & & 1.7547 & & 1.0100 & 1.0574 & 0.8777 & 0.9252 \\
\hline 91 & & & & 1.0514 & 1.0954 & 0.8842 & 0.9310 \\
\hline 92 & & & & 1.0654 & 1.1086 & 0.8807 & 0.9280 \\
\hline 93 & & & & 1.0836 & 1.1262 & 0.8653 & 0.9144 \\
\hline 94 & & & & 1.0987 & 1.1417 & 0.8616 & 0.9139 \\
\hline 95 & 1.1086 & 1.2364 & 1.0533 & 1.0519 & 1.0990 & 0.7451 & 0.8354 \\
\hline 96 & 1.0915 & 1.1953 & 1.0429 & 1.0211 & 1.0685 & 0.7105 & 0.8112 \\
\hline 97 & 1.0717 & 1.1440 & 1.0503 & 0.9704 & 1.0205 & 0.6535 & 0.7836 \\
\hline 98 & 1.0575 & 1.1195 & 1.0581 & 0.9415 & 0.9928 & 0.6292 & 0.7750 \\
\hline 99 & 1.0153 & 1.0664 & 1.0852 & 0.8724 & 0.9268 & 0.5877 & 0.7694 \\
\hline 100 & 0.9620 & 1.0082 & 1.0835 & 0.8114 & 0.8666 & 0.5653 & 0.7598 \\
\hline 101 & & 1.7020 & & 1.0126 & 1.0549 & 0.8668 & 0.9190 \\
\hline 102 & & & & 1.0462 & 1.0883 & 0.8803 & 0.9347 \\
\hline 103 & & & & 1.0709 & 1.1117 & 0.8704 & 0.9276 \\
\hline 104 & & 1.6503 & & 1.0834 & 1.1234 & 0.8644 & 0.9239 \\
\hline 105 & & 1.4624 & & 1.1168 & 1.1582 & 0.8587 & 0.9281 \\
\hline 106 & 1.1224 & 1.2700 & 1.0640 & 1.0784 & 1.1230 & 0.7638 & 0.8697 \\
\hline 107 & 1.0877 & 1.1850 & 1.0345 & 1.0132 & 1.0584 & 0.6950 & 0.8240 \\
\hline 108 & 1.0752 & 1.1562 & 1.0299 & 0.9852 & 1.0312 & 0.6689 & 0.8133 \\
\hline 109 & 1.0501 & 1.1103 & 1.0343 & 0.9294 & 0.9780 & 0.6267 & 0.8100 \\
\hline
\end{tabular}




\begin{tabular}{|c|c|c|c|c|c|c|c|}
\hline 110 & 1.0162 & 1.0700 & 1.0207 & 0.8841 & 0.9338 & 0.6052 & 0.8042 \\
\hline 111 & & 1.9194 & & 0.9651 & & 0.8416 & 0.8970 \\
\hline 112 & & 1.8269 & & 1.0172 & & 0.8736 & 0.9312 \\
\hline 113 & & 1.6323 & & 1.0577 & & 0.8771 & 0.9386 \\
\hline 114 & & 1.5624 & & 1.0706 & & 0.8724 & 0.9361 \\
\hline 115 & & 1.4736 & & 1.0886 & & 0.8540 & 0.9253 \\
\hline 116 & & 1.4420 & & 1.1041 & & 0.8499 & 0.9271 \\
\hline 117 & 1.1046 & 1.2382 & 1.0434 & 1.0578 & & 0.7384 & 0.8667 \\
\hline 118 & 1.0895 & 1.1992 & 1.0274 & 1.0268 & & 0.7077 & 0.8483 \\
\hline 119 & 1.0719 & 1.1488 & 1.0161 & 0.9764 & & 0.6624 & 0.8405 \\
\hline 120 & 1.0584 & 1.1244 & 1.0095 & 0.9475 & & 0.6456 & 0.8450 \\
\hline 121 & 1.0179 & 1.0715 & 0.9936 & 0.8789 & & 0.6264 & 0.8759 \\
\hline 122 & 0.9651 & 1.0130 & 0.9494 & 0.8183 & & 0.6175 & 0.8907 \\
\hline 123 & & 1.8481 & & 1.0516 & & 0.8933 & 0.9564 \\
\hline 124 & & 1.7189 & & 1.0624 & & 0.8866 & 0.9516 \\
\hline 125 & & 1.5384 & & 1.0763 & & 0.8646 & 0.9355 \\
\hline 126 & & 1.4809 & & 1.0840 & & 0.8540 & 0.9303 \\
\hline 127 & 1.0637 & 1.4022 & 1.0532 & 1.1034 & & 0.8353 & 0.9262 \\
\hline 128 & 1.0917 & 1.3768 & 1.0671 & 1.1148 & & 0.8271 & 0.9283 \\
\hline 129 & 1.1110 & 1.2980 & 1.0597 & 1.0961 & & 0.7811 & 0.9058 \\
\hline 130 & 1.1003 & 1.2505 & 1.0398 & 1.0668 & & 0.7480 & 0.8845 \\
\hline 131 & 1.0819 & 1.1829 & 1.0127 & 1.0145 & & 0.6949 & 0.8629 \\
\hline 132 & 1.0749 & 1.1591 & 1.0036 & 0.9903 & & 0.6757 & 0.8650 \\
\hline 133 & 1.0623 & 1.1260 & 0.9951 & 0.9436 & & 0.6542 & 0.8969 \\
\hline 134 & 1.0502 & 1.1080 & 0.9870 & 0.9166 & & 0.6551 & 0.9289 \\
\hline 135 & & 2.0081 & & 1.0696 & & 0.9150 & 0.9808 \\
\hline 136 & & 1.7026 & & 1.0742 & & 0.8861 & 0.9565 \\
\hline 137 & & 1.5961 & & 1.0769 & & 0.8708 & 0.9451 \\
\hline 138 & 1.0132 & 1.4494 & 1.0336 & 1.0842 & & 0.8423 & 0.9270 \\
\hline 139 & 1.0356 & 1.4004 & 1.0378 & 1.0889 & & 0.8289 & 0.9213 \\
\hline 140 & 1.0774 & 1.3291 & 1.0477 & 1.0951 & & 0.8007 & 0.9146 \\
\hline 141 & 1.0876 & 1.2924 & 1.0432 & 1.0857 & & 0.7789 & 0.9052 \\
\hline 142 & 1.0826 & 1.2190 & 1.0175 & 1.0447 & & 0.7266 & 0.8821 \\
\hline 143 & 1.0796 & 1.1911 & 1.0060 & 1.0234 & & 0.7046 & 0.8794 \\
\hline 144 & 1.0736 & 1.1532 & 0.9899 & 0.9822 & & 0.6735 & 0.8987 \\
\hline 145 & 1.0713 & 1.1408 & 0.9837 & 0.9626 & & 0.6683 & 0.9251 \\
\hline 146 & 1.0662 & 1.1236 & 0.9774 & 0.9230 & & 0.6829 & 1.0148 \\
\hline 147 & & 1.9965 & & 1.0928 & & 0.9256 & 0.9949 \\
\hline 148 & & 1.8216 & & 1.0843 & & 0.9021 & 0.9738 \\
\hline 149 & 0.9692 & 1.5657 & 1.0289 & 1.0778 & & 0.8609 & 0.9409 \\
\hline 150 & 0.9918 & 1.4786 & 1.0277 & 1.0774 & & 0.8433 & 0.9285 \\
\hline 151 & 1.0357 & 1.3673 & 1.0328 & 1.0830 & & 0.8138 & 0.9159 \\
\hline 152 & 1.0527 & 1.3253 & 1.0318 & 1.0811 & & 0.7968 & 0.9089 \\
\hline 153 & 1.0715 & 1.2546 & 1.0215 & 1.0639 & & 0.7559 & 0.8956 \\
\hline 154 & 1.0751 & 1.2244 & 1.0124 & 1.0485 & & 0.7338 & 0.8912 \\
\hline
\end{tabular}




\begin{tabular}{|c|c|c|c|c|c|c|}
\hline 155 & 1.0717 & 1.1732 & 0.9882 & 1.0091 & 0.6931 & 0.8956 \\
\hline 156 & 1.0731 & 1.1584 & 0.9808 & 0.9923 & 0.6818 & 0.9135 \\
\hline 157 & 1.0796 & 1.1460 & 0.9740 & 0.9625 & 0.6799 & 0.9891 \\
\hline 158 & 1.0883 & 1.1494 & 0.9747 & 0.9527 & 0.6950 & 1.0500 \\
\hline 159 & 0.9227 & 1.7880 & 1.0380 & 1.0862 & 0.8942 & 0.9688 \\
\hline 160 & 0.9504 & 1.6394 & 1.0281 & 1.0764 & 0.8686 & 0.9473 \\
\hline 161 & 0.9954 & 1.4453 & 1.0232 & 1.0735 & 0.8302 & 0.9219 \\
\hline 162 & 1.0165 & 1.3797 & 1.0221 & 1.0731 & 0.8130 & 0.9132 \\
\hline 163 & 1.0506 & 1.2937 & 1.0212 & 1.0701 & 0.7798 & 0.9033 \\
\hline 164 & 1.0600 & 1.2589 & 1.0151 & 1.0619 & 0.7603 & 0.8986 \\
\hline 165 & 1.0648 & 1.1981 & 0.9930 & 1.0307 & 0.7173 & 0.8936 \\
\hline 166 & 1.0666 & 1.1766 & 0.9822 & 1.0140 & 0.7001 & 0.9024 \\
\hline 167 & 1.0705 & 1.1502 & 0.9655 & 0.9823 & 0.6795 & 0.9517 \\
\hline 168 & 1.0801 & 1.1501 & 0.9638 & 0.9727 & 0.6840 & 1.0014 \\
\hline 169 & 0.9039 & 1.9247 & 1.0479 & 1.0961 & 0.9115 & 0.9852 \\
\hline 170 & 0.9558 & 1.5899 & 1.0187 & 1.0673 & 0.8517 & 0.9338 \\
\hline 171 & 0.9780 & 1.4789 & 1.0119 & 1.0620 & 0.8295 & 0.9181 \\
\hline 172 & 1.0235 & 1.3493 & 1.0164 & 1.0679 & 0.7999 & 0.9076 \\
\hline 173 & 1.0390 & 1.3025 & 1.0141 & 1.0648 & 0.7830 & 0.9031 \\
\hline 174 & 1.0569 & 1.2330 & 1.0018 & 1.0483 & 0.7446 & 0.8962 \\
\hline 175 & 1.0602 & 1.2038 & 0.9909 & 1.0349 & 0.7249 & 0.8980 \\
\hline 176 & 1.0548 & 1.1537 & 0.9606 & 0.9955 & 0.6866 & 0.9167 \\
\hline 177 & 1.0606 & 1.1449 & 0.9538 & 0.9832 & 0.6801 & 0.9499 \\
\hline 178 & 1.0894 & 1.1559 & 0.9563 & 0.9730 & 0.6953 & 1.0729 \\
\hline 179 & 0.9249 & 1.8488 & 1.0275 & 1.0769 & 0.8865 & 0.9604 \\
\hline 180 & 0.9490 & 1.6704 & 1.0100 & 1.0589 & 0.8540 & 0.9321 \\
\hline 181 & 0.9881 & 1.4325 & 1.0025 & 1.0536 & 0.8134 & 0.9063 \\
\hline 182 & 1.0115 & 1.3634 & 1.0064 & 1.0567 & 0.7996 & 0.9027 \\
\hline 183 & 1.0471 & 1.2796 & 1.0093 & 1.0603 & 0.7725 & 0.9022 \\
\hline 184 & 1.0557 & 1.2439 & 1.0024 & 1.0524 & 0.7539 & 0.9000 \\
\hline 185 & 1.0507 & 1.1773 & 0.9719 & 1.0168 & 0.7088 & 0.8987 \\
\hline 186 & 1.0469 & 1.1513 & 0.9547 & 0.9965 & 0.6904 & 0.9113 \\
\hline 187 & 1.0519 & 1.1280 & 0.9327 & 0.9669 & 0.6738 & 0.9886 \\
\hline 188 & 1.0694 & 1.1375 & 0.9350 & 0.9650 & 0.6830 & 1.0594 \\
\hline 189 & 0.9498 & 1.5749 & 0.9795 & 1.0276 & 0.8201 & 0.8987 \\
\hline 190 & 0.9653 & 1.4386 & 0.9742 & 1.0229 & 0.7977 & 0.8840 \\
\hline 191 & 1.0310 & 1.3377 & 1.0066 & 1.0580 & 0.7923 & 0.9019 \\
\hline 192 & 1.0488 & 1.2951 & 1.0091 & 1.0610 & 0.7803 & 0.9038 \\
\hline 193 & 1.0606 & 1.2244 & 0.9950 & 1.0454 & 0.7433 & 0.9018 \\
\hline 194 & 1.0556 & 1.1894 & 0.9769 & 1.0265 & 0.7202 & 0.9022 \\
\hline 195 & 1.0178 & 1.1085 & 0.9175 & 0.9612 & 0.6651 & 0.9065 \\
\hline 196 & 1.0212 & 1.0986 & 0.9066 & 0.9471 & 0.6592 & 0.9561 \\
\hline 197 & 0.9332 & 1.6049 & 0.9324 & 0.9778 & 0.7899 & 0.8603 \\
\hline 198 & 0.9947 & 1.3927 & 0.9744 & 1.0242 & 0.7895 & 0.8796 \\
\hline 199 & 1.0338 & 1.3526 & 0.9992 & 1.0505 & 0.7947 & 0.8970 \\
\hline
\end{tabular}




\begin{tabular}{|l|l|l|l|l|l|l|l|}
\hline 200 & 1.0707 & 1.2817 & 1.0132 & 1.0667 & & 0.7776 & 0.9090 \\
\hline 201 & 1.0747 & 1.2462 & 1.0057 & 1.0586 & & 0.7594 & 0.9094 \\
\hline 202 & 1.0513 & 1.1666 & 0.9616 & 1.0122 & & 0.7064 & 0.9025 \\
\hline 203 & 1.0219 & 1.1160 & 0.9233 & 0.9710 & & 0.6730 & 0.8994 \\
\hline 204 & 0.9719 & 1.0375 & 0.8513 & 0.8935 & & 0.6293 & 0.9612 \\
\hline 205 & 0.9692 & 1.3535 & 0.9276 & 0.9743 & & 0.7584 & 0.8385 \\
\hline 206 & 1.0708 & 1.3401 & 1.0119 & 1.0644 & & 0.7992 & 0.9060 \\
\hline 207 & 1.0914 & 1.3084 & 1.0243 & 1.0786 & & 0.7942 & 0.9184 \\
\hline 208 & 1.0933 & 1.2406 & 1.0093 & 1.0638 & & 0.7584 & 0.9177 \\
\hline 209 & 1.0724 & 1.1914 & 0.9805 & 1.0341 & & 0.7261 & 0.9105 \\
\hline 210 & 0.9741 & 1.0531 & 0.8713 & 0.9191 & & 0.6347 & 0.8673 \\
\hline
\end{tabular}

